\title{
Elasticity and Morphology of Wet Fiber Networks
}

\author{
Dissertation \\ zur Erlangung des mathematisch-naturwissenschaftlichen Doktorgrades \\ "Doctor rerum naturalium" \\ der Georg-August-Universität Göttingen
}

vorgelegt von

Jann Ohle Claussen

aus Leer

Göttingen, 2011 
Referent: Stephan Herminghaus

Korreferent: Marcus Müller

Tag der mündlichen Prüfung: 
The world that is the most perfect [is] the one that is at the same time the simplest in hypothesis and the richest in phenomena

(Gottfried Leibniz, Discourse on Metaphysics, 1686)

Hey! Ho! Let's Go!

(Dee Dee Ramone, 1976) 


\section{Abstract}

Fibrous materials are very commonplace in current technological applications. The study of their mechanics is an interesting topic that has previously been inspired by applications in the textile and paper industries, but also by modern composite materials. In many applications, humidity of the ambient atmosphere leads to condensation of liquid in a fiber network. Once liquid interfaces form, capillary effects lead to internal mechanical stresses.

In this work, we investigate the effects of capillary stresses on disordered, two-dimensional networks of elastic fibers. To that end, we first study the morphologies and induced stresses of a liquid bridge forming between two cylindrical fibers. Then, we introduce a two-dimensional model system for disordered fiber networks with liquid interactions taking place at fiber crossings. After an overview of the topological properties of such a model network, we introduce a method for computer simulation of an elastic network in the presence of a wetting liquid that can accumulate in the fiber interstices and thereby deform the network. We test the model by comparison with analytical theory in a simple test case, and finally we present computer experiments characterizing the network deformation as a function of the most important model parameters, the fiber density and the capillary interaction strength. 


\section{Contents}

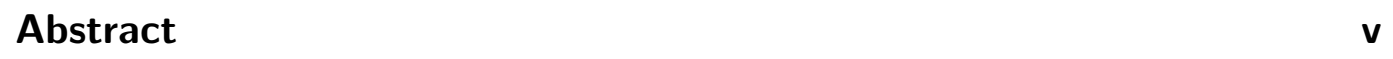

\begin{tabular}{lll}
\hline 1 & Introduction & 1
\end{tabular}

\begin{tabular}{|lll}
2 & Wetting between Cylindrical Surfaces & 7
\end{tabular}

$2.1 \quad$ Wetting Liquids on Solid Substrates . . . . . . . . . . . . . 8

$2.2 \quad$ Analytical Description of Wetting Between Cylinders . . . . . 17

2.3 Numerical Computation $\ldots \ldots \ldots \ldots$

3 Crosslinked Fiber Networks 37

3.1 Crosslinkers . . . . . . . . . . . . . . . . . . . . . . 39

3.2 Random Fiber Networks: Mikado Models . . . . . . . . . . . . 40

3.3 Topological Rigidity . . . . . . . . . . . . . . . . . . . . . . 44

3.4 The Pebble Game . . . . . . . . . . . . . . . . . . . . . . . . 50

3.5 Rigidity Percolation of Diluted Mikado Networks . . . . . . . 53

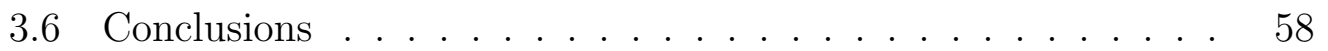

4 Wet Fiber Networks $\quad 61$

4.1 Discrete Element Model for Elastic Fibers . . . . . . . . . . . 62

4.2 Liquid Bridges as Mobile Crosslinks . . . . . . . . . . . . . . . 69

4.3 Numerical Integration of Equations of Motion . . . . . . . . . 89

4.4 Capillary Adhesion of Fibers . . . . . . . . . . . . . . . . . . 92

4.5 Network Deformation by Weak Liquid Bridges . . . . . . . . . . 99

4.6 Observations about Networks Deformed by Strong Liquid Bridges 102

4.7 Morphological Transitions . . . . . . . . . . . . . . . . . . 104

4.8 Elastic Response . . . . . . . . . . . . . . . . . . . . . . . . 113

5 Conclusions and Outlook 115

5.1 Conclusions . . . . . . . . . . . . . . . . . . . . . . . 115

5.2 Outlook .............................. 117 


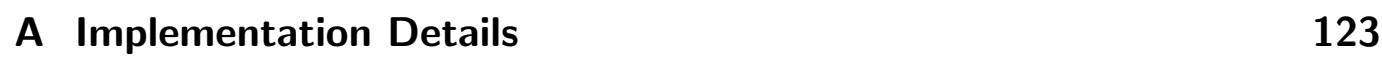

A.1 Data Structures . . . . . . . . . . . . . . . . . . . . . . . . 123

A.2 Tracking of Bridges . . . . . . . . . . . . . . . . . . . . . . 125

A.3 Detection of Elementary Loops . . . . . . . . . . . . . . . . . 129

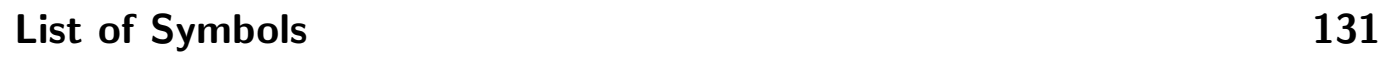

\begin{tabular}{lr}
\hline Bibliography & 142
\end{tabular}

\begin{tabular}{ll}
\hline Curriculum Vitae / Lebenslauf & 143
\end{tabular}

\begin{tabular}{ll}
\hline Danksagung (acknowledgements) & 145
\end{tabular} 


\section{Chapter 1}

\section{Introduction}

Many materials in technology and biology can be described as networks of fibers. Especially in textiles and paper, the influence that a wetting liquid has on these materials can be observed in everyday life. Typical non-woven fabrics, i.e., disordered fibrous materials, such as wool, cotton or leather, but also human or animal hair, wick up liquids and form concentrated bundles of wet fibers.

But also the properties of artificial soft porous media, as for example solid foams made of elastic polymeric materials, or the disordered assemblies of fibers found in aerosol filters, vary strongly between the wet and the dry states. Wetting of macroscopic fiber networks is relevant to many industrial production processes and applications, in particular in the textile industry. A large number of reinforced composite materials are based on elastic fiber networks. To ensure structural integrity of these composite materials, the fibers have to be glued together by binder materials while the remaining pore space is filled with a solid material. In both cases, the wetting properties of the fibers by the fluid binder and filler materials are important parameters in the production process and have to be carefully controlled.

Many properties of a fiber network undergo large changes when it is exposed to a wetting liquid. The mechanical response of an elastic fiber network to compression or shear, for example, changes noticeably between the dry and the wet states. Attractive capillary forces enhance inter-fiber friction, while lubrication of the fiber contacts may result in the opposite effect. An additional amount of work is dissipated in discontinuous transitions between different locally stable configurations of the wetting liquid and the fibers. The magnitude of this elastocapillary hysteresis will have a large impact on the mechanics of the network, e.g., the shear or compression moduli, and one may expect to find a number of liquid saturation regimes related to different 


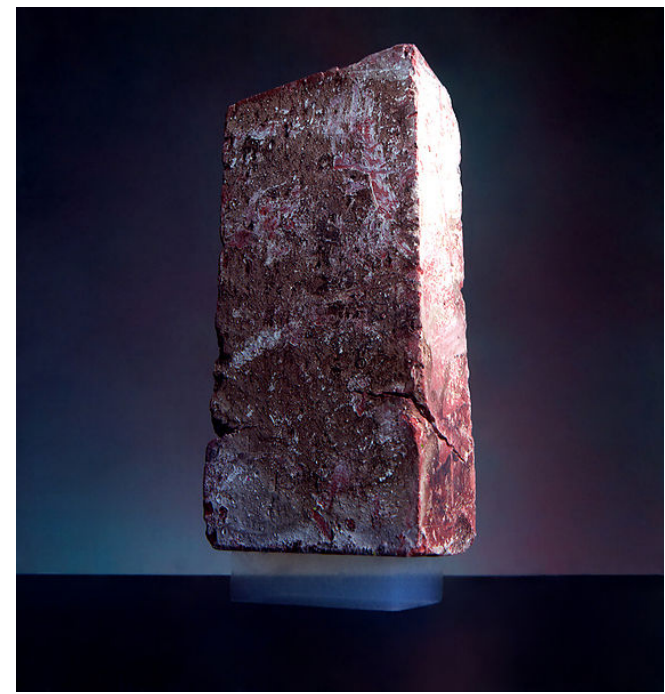

(a) Mechanical strength

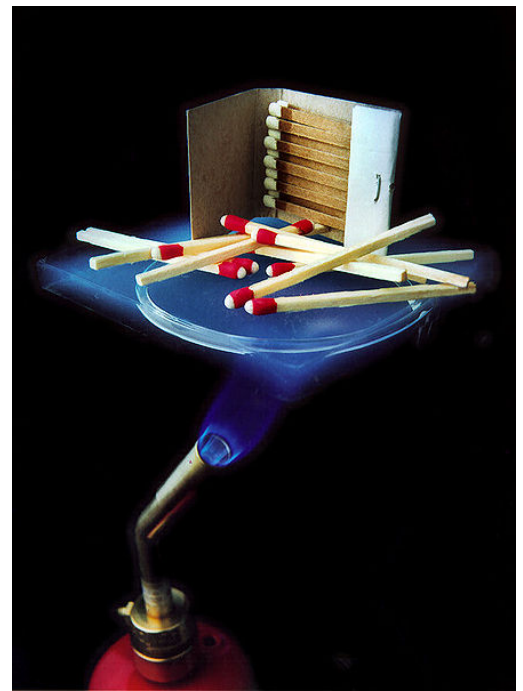

(b) Thermal insulation

Figure 1.1: A slab of aerogel, supporting a $2 \mathrm{~kg}$ brick (a) and thermally insulating unlit matches from a flame (b) (images by NASA)

dissipation mechanisms.

One type of material where capillarity is known to have destructive effects is aerogel (see figure 1.1). This consists of the crosslinked fibers of a gel, which remain after the solvent of the gel has been replaced with gas. It is an extremely light material that can nevertheless support large forces and that acts as a very efficient thermal insulator.

The process of removing the liquid solvent from the gel is very critical in the production of aerogels, since the appearance of liquid menisci leads to capillary stresses that will destroy the material. For the same reason, aerogels are very sensitive to ambient humidity.

A well-studied example of materials where the effect of wetting and capillary interaction has been studied is that of wet granulates, such as sand on the beach. Here, it is well known that the addition of liquid can change the mechanical properties dramatically. This fact has been characterized in many experiments such as those presented in $\left[\mathrm{FGH}^{+} 05\right]$. In the case of wet granulates, a simple model of the interaction between pairs of spheres by means of a liquid bridge has lead to deeper insights into the behavior of the material (see [Her05]). The model consists of an attractive force that acts once two grains have collided and ceases to act once the resulting liquid bridge has 
ruptured at a certain separation of the grains.

In this work, we want to apply a similarly simple model to elongated objects, specifically cylindrical fibers. Since we impose cylindrical symmetry, the geometry near the contact point can be described simply by the crossing angle of the fibers. In systems of cylindrical fibers, liquid will condense at the crossing points and form filaments between the fibers. These exert torques on the crossing fibers and tend to align them. New filaments form whenever fibers touch, and filaments are destroyed in rupture events similar to those described above for wet granulates. Since fibers are objects with a large aspect ratio, they will undergo noticeable elastic deformations in response to capillary forces.

The balance between capillary forces exerted by a liquid interface and elastic forces exerted by the substrate the liquid adheres to leads to what is called elastocapillary effects. A very instructive example of such effects can be seen when a liquid droplet is deposited on a thin elastic sheet. Py and coworkers observed in $\left[\mathrm{PRD}^{+} 07\right]$ that capillary forces lead to a wrapping of the sheet around the droplet into various shapes. The authors called this capillary origami. In [PnBR10, it is shown that by applying an electric field to the droplet, this folding process can be controlled.

In biology, elastocapillarity is one of the ways by which plant leaves such as those of the indian cress (Tropaeolum majus L.) achieve superhydrophobicity. In [OH04], Otten and Herminghaus describe how hairs on the leaves' surface deform under capillary forces induced by the liquid interface of a water droplet. This elastic deformation leads to an effective repulsion of the droplet from the surface of the leaf itself. In this way, the droplet is prevented from wetting the leaf.

Elastocapillary effects are known to lead to structure formation in materials composed of elastic fibers in contact to a wetting liquid: While pulling a comb of elastic lamellae out of a bath of silicone oil, Bico observed in [BRMB04 that the lamellae arranged into hierarchical bundles. In [LFXZ07], the authors address the interplay between the elastic deformation of fibers or hairs anchored to a surface and the capillary adhesion of these hairs into pairs or larger bundles. In [ $\left.\mathrm{PBB}^{+} 07\right]$, further experiments are presented using brushes of flexible glass and polystyrene fibers in a three-dimensional arrangement. Similarly, carbon nanotube forests covering a surface are observed to collapse into bundled cell-like structures when a liquid is evaporated from the material (see $\left.\left[\mathrm{CWC}^{+} 04\right]\right)$. Also, polymer nanobristles have been observed to 
self-organize into helical bundles by elastocapillary coalescence [PKMA09].

The problem of a liquid invading a disordered medium is surveyed in an extensive review article by Mikko Alava and coworkers in [ARD04.

In [BLL $\left.{ }^{+} 99\right]$, Beckham et al. measure the moisture content in fiber mats by MRI, and observe the retention of water during drying. Nomura et al. studied capillary condensation in Aerogels in [NMYO06]. They used ${ }^{4} \mathrm{He}$ as the wetting liquid, since it has very low surface tension, and therefore avoids the problem capillary stresses.

Capillary stresses induced by the liquid and the associated fiber deformations are not specifically addressed in these works, although it is clear that internal torques induced by capillarity must change the fiber orientations.

A preliminary account of fiber rearrangement effects induced by capillarity can be found in Skelton's work Ske75a], where the question of hysteresis of the liquid saturation during a cyclic wetting and drying of fibrous filters is addressed.

Capillarity-induced fiber rearrangements also have an effect on the mechanics of a fiber network. Already in 1952, Cox and coworkers showed in an analytic study that the orientation of fibers influences the strength of paper and other fibrous materials significantly Cox52]. In a more recent study, Chatterjee examines the effect of statistical heterogeneities in non-uniform fiber arrangements on the mechanical properties, stressing that the elastic moduli depend sensitively on the mean pore size [Cha10, Cha11. As we will see in chapter 4, formation and growth pores is one of the major effects capillary stresses have on the morphology of elastic networks.

In this work, we focus on cylindrical fibers as the constituents of a disordered elastic medium interacting with a wetting liquid. As well as being a good model for textile fibers, polymers, and fibers in many other technical applications, cylinders are also the simplest geometrical objects that are elongated and can therefore react to capillary stresses by rotational motions as well as translations. For this reason, cylinders provide a minimal model for fibers, similar to spherical beads used to model granulates.

We are interested in disordered arrangements of such cylinders in a mechanical system, and therefore use fiber networks as our model system. For simplicity, we will also neglect surface roughness on the cylinders in our model. In view of the background of fiber networks of the type found in textiles, the cylinders should be thought of as having a radius ranging from some $10 \mu \mathrm{m}$ to a few millimeters, and lengths in the order of centimeters. In this size range, 
formation of droplets and other liquid morphologies attached to the fibers can be observed.

In nature, condensation of liquids on fibers can be observed in the droplets forming on spider webs in the mornings. Zheng et al. have studied water collection on spider silk, as an example system for condensation on micrometric fibers $\left[\mathrm{ZBH}^{+} 10\right]$.

In technology, capillary condensation in fiber systems occurs, for example, in aerosol filters. Liew and coworkers studied the water uptake of aerosol filters, and worked out consequences to their flow resistance and filtering efficiency in [LC85, CL89], concentrating on micrometric steel wires that do not deform significantly through capillary stresses.

Different states of a wetting liquid approaching a crossing point of two fibers have been investigated by Gilet and coworkers [GTV09], who were interested in designing systems of fibers to guide droplets in a novel digital microfluidics setup.

In this work, our chief interest is in the effect that a liquid has at the contact points of fibers in a disordered network, where it will form filaments due to capillary condensation and exert mechanical forces and torques on the elastic fibers. To this end, we extend the work of Princen Pri69a, Pri69b, Pri70] to describe the shape of liquid filaments between crossed cylinders, and use numerical methods to derive a model for the capillary torque they exert, in chapter 2 .

Later on, in chapter 3, we will introduce a two-dimensional model system for disordered fibrous materials, which allows us to simulate the effect of capillary interactions taking place at the crossing points of fibers.

The first part of chapter 4 is devoted to the specifics of the interaction model. In this model, the liquid bridges are viewed as mobile crosslinks that appear by condensation at the crossing points and move along with the crossing points as the network deforms. In this way, the liquid is transported through the system and accumulates in regions of strongly bundled fibers.

A similar concept to this is the introduction of reversible crosslinks that can form when fibers approach each other, and can rupture when they experience a large strain. This has been used to model crosslinkers in biological networks, but has the very significant difference that while these crosslinks exist, they are not freely slidable, i.e., they act just like a fixed crosslink, and they also do not exert aligning torques. In this field, $\mathrm{Xu}$ et al. [XHL ${ }^{+}$] ] have investigated solutions of polymers with reversible crosslinks and found shear-thickening 
effects. Also, Åström et al. [̊KVK08] have investigated dense networks with reversible crosslinks and found that on cyclic compression, these form bundles, not dissimilar to those that are found in this work, in the results of chapter 4 . 


\section{Chapter 2}

\section{Wetting between Cylindrical Surfaces}

Interfacial tension may induce an attractive interaction between two objects in contact to a liquid-fluid interface. As a prominent example, these capillary forces give rise to the mechanical stability of wet sand: The presence of numerous small water bridges forming at the contact points between grains causes a noticeable cohesion which allows one to build sand castles on the beach. Completely dry or wet mixtures of sand and water show no cohesive force because of the absence of interfaces.

Analogously, it is clear that capillary interactions are a key point to understanding the properties of wet fibrous materials as well. A theoretical model of the capillary forces and torques explains the mechanical response of many systems ranging from wet non-woven fabrics, paper and tissues to fiber-based composite materials and wet hair. In particular, the mechanical stability of areogels is strongly affected by the ambient moisture.

In a good approximation, one may consider sand grains as being spherical particles. Therefore, study of capillary forces between spherical bodies in the context of wet soils dates back to the middle of the $19^{\text {th }}$ century. However, capillary interaction between strongly elongated bodies, in particular cylindrical surfaces as a model for fibers, has been rarely addressed.

A study of the Rayleigh-Plateau instability leading to the separation of a liquid film on a cylindrical fiber into droplets has been done in 1975 by Roe [Roe75]. Later, McHale described a bistability of liquid morphologies on a fiber (a barrel state surrounding the fiber and a clamshell state shifted to one side) in $\mathrm{McH} 02$. Experiments on this phenomenon have been performed by Lorenceau et al. in LCQ04. Applications to fibrous materials have also been considered in the literature. Mullins and coworkers observed the way in which 
droplets are captured by fibers in an aerosol filter [MBA04], and the influence of the relative orientation of its fibers on the flow of droplets through such a filter [Mul04]. Already in 1975, Skelton measured capillary forces between fibers during the process of wetting an aerosol filter [Ske75b].

In this chapter, we will first introduce the reader to the fundamental principles of wetting on solid substrates. The concept of interfacial and surface tension and its thermodynamic foundations will be explained in section 2.1 and then applied to the calculation of the equilibrium shapes of liquid bridges held between two cylindrical surfaces in section 2.2. At least two types of bridges are encountered for small crossing angles and separation distances: On the one hand, these are elongated liquid structures called filaments and on the other hand, localized, droplet-like shapes.

To describe the shape of elongated filaments we will apply an analytical model which is then compared to the results of numerical calculations based on energy minimizations. In both approaches we will derive the capillary torques as a function of the tilt angle which shows a power law behavior at small angles. The exponent of this power law depends on the volatility of the liquid: For non-volatile liquids, the liquid volume of a bridge can be regarded as constant, while the volume of small liquid bridges of volatile liquids is controlled by the vapor pressure.

The findings of this chapter will enable us to formulate an effective model for capillary interaction that can be further employed in simulations of elastic fiber networks, as we will show in chapter 4

\subsection{Wetting Liquids on Solid Substrates}

Wetting of solid substrates by a liquid is a commonplace phenomenon that has implications for many technical applications and biological processes. An example that springs immediately to mind is the coating of frying pans to make them non-sticking.

In biology, the lotus leaf uses a microstructured surface to induce superhydrophobicity, which helps the leaves to clean in rain, since the water droplets pick up dirt particles but no not stick to the leaf itself. This same effect is now being used to manufacture self-cleaning surfaces.

In the following sections, we will outline the fundamental physics behind wetting that determine the shape of a liquid phase that is in coexistence with an immiscible fluid phase and in contact to a rigid and ideally smooth sub- 


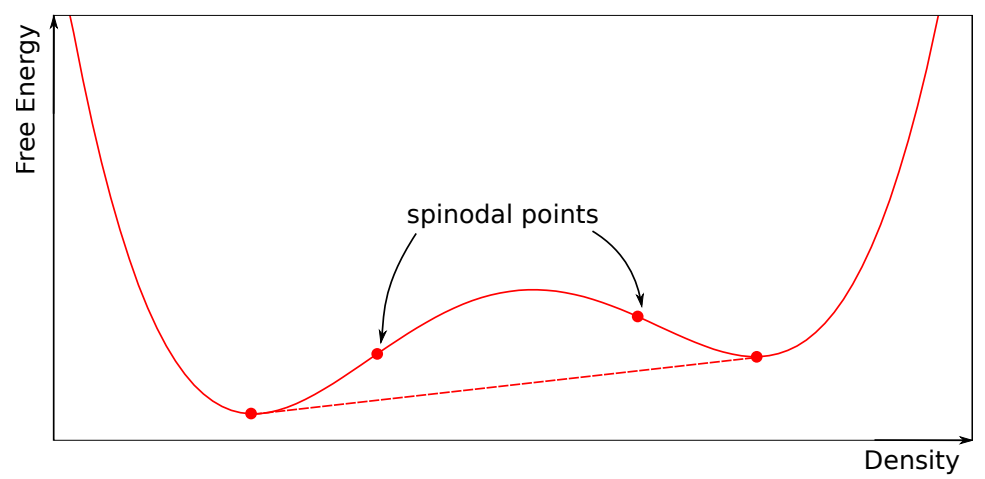

Figure 2.1: Free energy of a liquid vapor below the critical point as a function of density

strate. Then we apply these principles to the specific geometry of two cylinders touching in a point. Finally, we present results of numerical minimizations of the interfacial free energy in this geometry to determine the capillary forces a liquid filament will exert on the cylinders.

We will assume throughout our analysis that the surfaces under consideration are perfectly smooth and rigid, i.e., there is no microstructure that could cause pinning of the contact line or contact angle hysteresis. The analysis of droplets and filaments on rough cylinders is beyond the scope of this work.

\subsubsection{Thermodynamic Aspects}

We will now introduce the basic thermodynamics involved in the coexistence of a liquid and its vapor, which is the foundation of wetting phenomena and liquid-fluid interfaces.

Consider a container of volume $V_{0}$ with a solid substrate as walls, filled with vapor (or with a binary liquid mixture). The solid and vapor phase will be called $(s)$ and $(v)$, respectively, in the following.

Below a critical temperature $T_{c}$, the free energy of the vapor as a function of particle density $\rho$ takes on a shape similar to that depicted in figure 2.1. where there exists a double tangent connecting two local minima of the free energy, with the curve of the free energy in between lying completely above the double tangent. In such a case, the system will decompose into a highdensity liquid phase $(l)$ and a low-density vapor phase $(v)$ in such a way that the total free energy of the two phases takes on a value on the double tangent. 


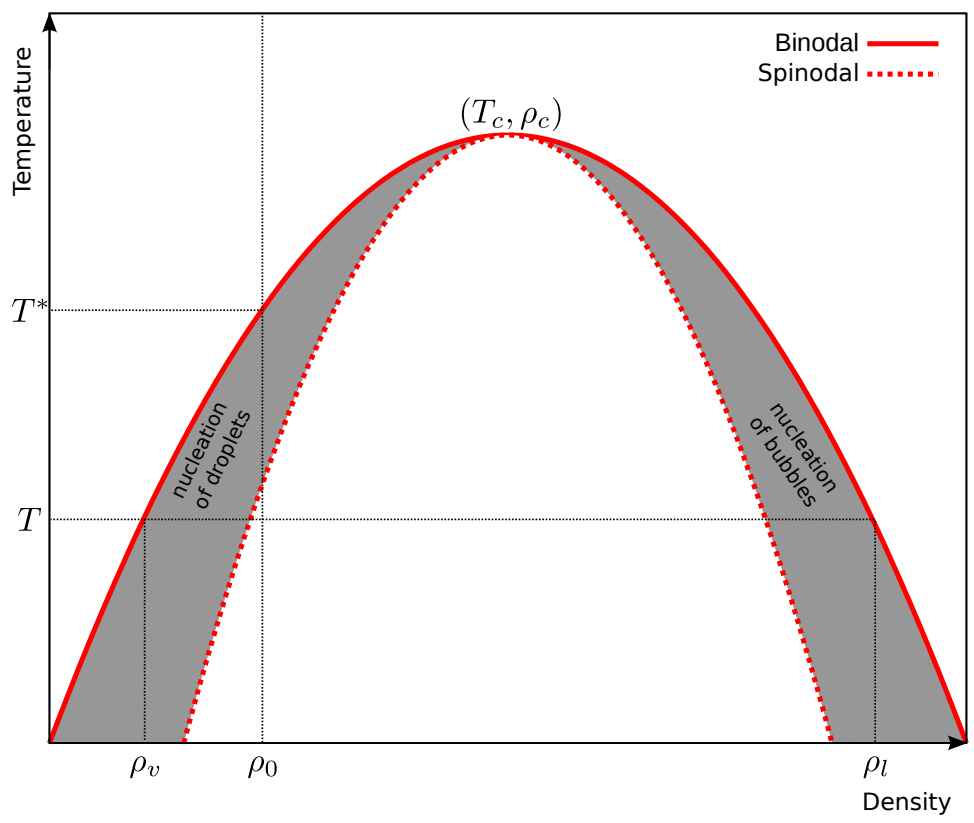

Figure 2.2: Phase diagram of a liquid vapor. Shown are the binodal and spinodal lines, the temperature range of metastability at a given particle density, and the decomposition ratio at $T$ according to the lever rule

Figure 2.2 shows a typical phase diagram of a liquid vapor, with the abscissa denoting the particle density $\rho$, and the ordinate temperature $T$. The phase diagram of a binary liquid with a miscibility gap has a similar shape, when one replaces $\rho$ with the particle composition. The solid line, the binodal, is the liquid-vapor coexistence line, which is constructed by the local minimum points of the free energy of a phase separating system. Above this line, the homogeneous vapor phase is thermodynamically favorable, while below it, the global minimum of the bulk free energy is a phase-separated state. The dashed line, the spinodal, marks the area where the homogeneous phase is absolutely unstable. It is defined as the line of vanishing second derivative of the free energy with respect to density. Inside of the spinodal, the homogeneous phase will always decompose into a mixed phase throughout the whole system. The shaded area between the spinodal and the binodal marks the area of metastability of the homogeneous phase. Within it, nucleation and growth of the minority phase can take place. The two boundaries meet at the critical point $\left(T_{c}, \rho_{c}\right)$, where all three phases coexist. 
Denoting the total number of particles in the container by $N_{0}$, the initial particle density $\rho_{0}=N_{0} / V_{0}$ determines the temperature $T^{*}$ where phase separation starts. At a given temperature $T<T^{*}$, the decomposition is governed by the lever rule: The total volumes $V_{l}$ and $V_{v}$ of phases $(l)$ and $(v)$, respectively, are given by the particle densities $\rho_{l}$ and $\rho_{v}$ of the binodal at temperature $T$ :

$$
\left(\rho_{0}-\rho_{l}\right) V_{l}=\left(\rho_{v}-\rho_{0}\right) V_{v}
$$

The two separated phases are in equilibrium with each other. The total differential $\mathrm{d} F$ of the free energy in this ensemble is a sum over that of the two phases:

$$
\mathrm{d} F=S \mathrm{~d} T+\mu_{l} \mathrm{~d} N_{l}-p_{l} \mathrm{~d} V_{l}+\mu_{v} \mathrm{~d} N_{v}-p_{v} \mathrm{~d} V_{v}
$$

Since the number of particles $N_{0}=N_{l}+N_{v}$ and the total volume $V_{0}=V_{l}+V_{v}$ are fixed, variation of the free energy of the system to find a minimum leads to the conditions of equal chemical potentials $\mu_{l}=\mu_{v}$ and equal pressures $p_{l}=p_{v}$

\subsubsection{Interfacial Energy}

In the metastable coexistence regime, formation of the minority phase occurs by nucleation, i.e., the appearance and growth of liquid droplets or vapor bubbles, respectively.

For small droplets and bubbles, interfacial contributions in the free energy play a significant role. The free energy to be gained by enlarging the nucleating phase out of the metastable phase can be of the same order of magnitude as the energy needed for the associated enlargement of the interface. Thus, there is an energy barrier to be crossed for a phase transition to take place.

At the interface between the liquid and vapor phases, the particle density has to change continuously between the equilibrium values of the two bulk phases. The associated free energy penalty depends on the density profile of the interface. To determine this density profile and the associated excess free energy of the interface, one needs to consider additional contributions related to density gradients [Saf03] (see also figure 2.1). This excess free energy is extensive in the interfacial area. It is easy to see that it must be positive, since if a droplet could decrease its free energy by enlarging its interface, the minimum energy state would be that where all particles belong to the 
interface, i.e., a homogeneous mixture. This contradicts the assumption that the homogeneous state is thermodynamically unstable.

One can express these additional contributions in terms of interfacial tensions $\sigma_{i j}, i, j \in\{s, l, v\}$ that give the areal densities of the free energy of the interfaces between phases $i$ and $j$. The $\sigma_{i j}$ correspond to work that has to be done on the system to change the interfacial areas $A_{i j}$. Equation $(2.2)$ then becomes:

$$
\mathrm{d} F=S \mathrm{~d} T+\mu_{l} \mathrm{~d} N_{l}-p_{l} \mathrm{~d} V_{l}+\mu_{v} \mathrm{~d} N_{v}-p_{v} \mathrm{~d} V_{v}+\sigma_{l v} \mathrm{~d} A_{l v}+\sigma_{s l} \mathrm{~d} A_{s l}+\sigma_{s v} \mathrm{~d} A_{s v}
$$

This is the differential of the total free energy of the system. It is to be noted that the volumes $V_{i}$ and areas $A_{i}$ are not independent, since they are coupled by the particular shape the interface takes on. Therefore, the free energy must be regarded as a functional of the shape. This implies that in equilibrium, the pressures $p_{l}$ and $p_{v}$ need not be identical anymore. The chemical potentials $\mu_{i}$, however, are equal in thermodynamical equilibrium.

At a given volume, a free droplet will then always minimize its interfacial area, so that it acquires a spherical shape. In the general case of a droplet wetting a solid substrate, the minimal shapes depend on the geometry of the substrate. In contrast to the case of the free droplet, a multitude of minimal shapes is possible, although transitions between these minima is typically associated with free energy barriers.

A full description of the free energy of the liquid would need to take the energy of particles on the three-phase contact line into account as well. Line tension terms are commonly neglected, however. The tension $\tau$ of the onedimensional contact line would give a contribution of $\tau \mathrm{d} L$ proportional to the line's length $L$. However, when the droplets are larger than the typical length $\lambda_{\tau}=\tau / \sigma_{l v}$, line tension contributions to the free energy are insignificant.

Gravitational contributions to the free energy can in some cases also be important. Comparing surface- and gravitational energies gives rise to another length scale called the capillary length:

$$
\lambda_{c}=\sqrt{\frac{\sigma_{l v}}{\rho g}} .
$$

As long as the typical dimensions $\lambda$ of a liquid droplet satisfy $\lambda_{\tau} \ll \lambda \ll \lambda_{c}$, line tension effects and gravitational effects do not play a role. For water at standard conditions, $\lambda_{c} \approx 3.8 \mathrm{~mm}$, and $\lambda_{\tau}$ is on the order of nanometers. Therefore, we will ignore both these effects in the rest of this work. 


\subsubsection{The Volume- and Pressure- Ensembles}

It is useful to distinguish two different thermodynamical scenarios when considering a wetting liquid on a surface: The ensembles of constant vapor pressure and of constant liquid volume.

The first ensemble applies to the experimental situation of small droplets of a volatile liquid in chemical equilibrium with its vapor phase.

In that case, the chemical potential, and in consequence the pressure of the liquid and vapor phase, is controlled externally. As a consequence, the difference in pressures between the liquid and vapor phases is also determined by the prescribed chemical potential.

Equilibrium states in this ensemble are stationary points of the grand canonical free energy

$$
\Omega=F-\mu N=\mathcal{F}-\Delta p V_{l}+p_{v} V_{0},
$$

where $\mathcal{F}=\sigma_{l v} A_{l v}+\sigma_{s l} A_{s l}+\sigma_{s v} A_{s v}$ is the interfacial part of the free energy. The last term in equation (2.5) is a constant.

In this ensemble, certain configurations are unstable with respect to exchange of particles. For example, in the following section we will see that the excess pressure of small droplets is determined by their interfacial curvature. Therefore, all droplets in the system will equilibrate to equal mean curvatures by exchange of particles through the vapor phase, since the vapor pressure determines the equilibrium curvature.

The ensemble of constant volumes corresponds to situations where the liquid is incompressible and non-volatile, meaning that exchange of particles between liquid and vapor is slow on experimentally relevant timescales. If that is the case, the volume of liquid in each drop is a conserved quantity in the system, and can be seen as a constraint in minimizing the free energy to derive the liquid shapes. Since the droplet volume is constant, only the interfacial parts of the free energy need to be considered for minimization. The functional to minimize is then given by

$$
\mathcal{F}^{\prime}=\mathcal{F}-\Delta p V_{l}
$$

Here, $\Delta p$ is a Lagrange multiplier that has to be chosen such that the volume constraint is satisfied. It is equal to the excess pressure that the liquid will have in mechanical equilibrium.

The sets of stationary points of the functionals $(2.5)$ and $(2.6)$ are identical. Owing to the additional constraint, the set of local minima, i.e., of stable 
shapes, is larger in the second case. For example, a spherical droplet of a small fixed volume is always stable in the volume ensemble. However, in the pressure ensemble, the same droplet, in chemical equilibrium with the ambient vapor phase, represents an unstable saddle point of $\Omega$, and will either evaporate or grow.

\subsubsection{Mechanical Equilibrium}

While equation (2.3) gives the conditions for thermodynamic equilibrium, one has to consider the balance of stresses to identify those liquid morphologies that are mechanically stable. First we consider the condition of mechanical equilibrium at the fluid-fluid interface:

\section{The Law of Laplace}

Laplace remarked that in mechanical equilibrium, the surface tension of a spherical droplet, or of any curved fluid interface, would make the droplet collapse unless it was counteracted by an excess pressure $\Delta p$ within the droplet. In general, the work of increasing the interfacial area by a small amount $\mathrm{d} A_{l v}$ must be counteracted by the work needed to increase the volume by $\mathrm{d} V_{l}$, so that $\Delta p \mathrm{~d} V_{l}=\sigma \mathrm{d} A_{l v}$.

Applying this to a surface element with the two principal radii of curvature $R_{1}$ and $R_{2}$, one can obtain the pressure difference:

$$
\Delta p=\sigma_{l v}\left(\frac{1}{R_{1}}+\frac{1}{R_{2}}\right)=2 \sigma_{l v} H .
$$

Here, $H$ is the mean curvature of the surface. The pressure difference $\Delta p$ is called the Laplace pressure.

Since the pressure in one phase will be homogeneous in equilibrium, a direct consequence of this is that the fluid-fluid interface in mechanical equilibrium is a surface of constant mean curvature.

\section{Young's Condition}

Young [You05] considered the equilibrium of forces at the three-phase contact line of a sessile droplet, that is, a droplet sitting on a flat substrate (see fig. 2.3). Each of the three interfacial tensions results in a force that will strive to minimize the corresponding interfacial area, and is therefore tangential to that interface. In order for such a droplet to be stable against movement of the 


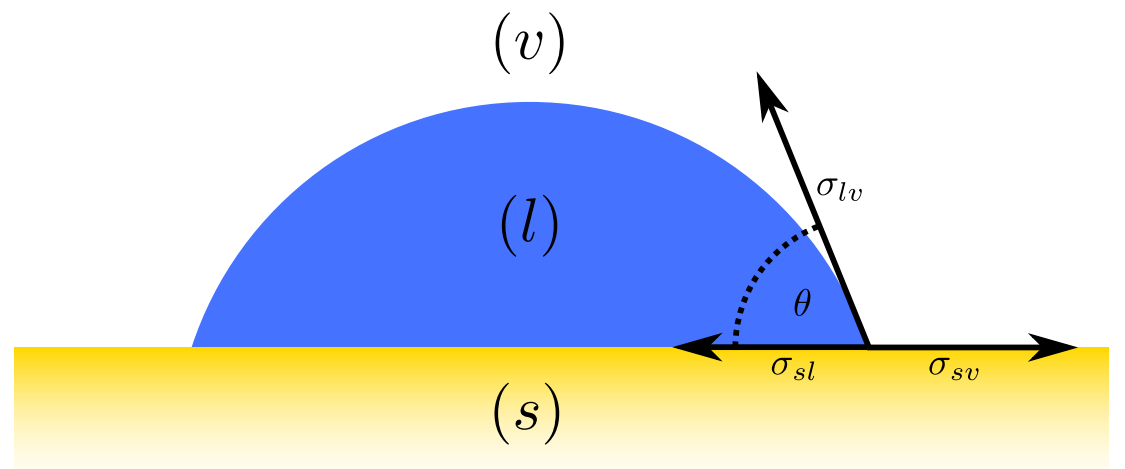

Figure 2.3: Balance of forces at the three-face contact line of a sessile droplet

contact line on the solid surface, the projections of the forces onto the surface must balance. The angle at which the $(l v)$ interface and the $(s l)$ interface meet is called the contact angle $\theta$, and it is fixed by the condition of force balance:

$$
\sigma_{s v}=\sigma_{s l}+\sigma_{l v} \cos \theta .
$$

This is Young's equation for the contact angle. It can only be fulfilled with a finite $\theta \in(0, \pi)$ when $\sigma_{s v}<\sigma_{s l}+\sigma_{l v}$ and $\sigma_{s l}<\sigma_{l v}+\sigma_{s v}$. If the first of these conditions is not fulfilled, the contact line spreads outward indefinitely until the liquid forms a thin film on the whole substrate. This is called complete wetting. Violation of the second condition leads to complete retraction of the contact line until the spherical droplet touches the surface at only one point; this is called a completely non-wetting scenario.

On real surfaces, microscopic roughness will mean that the macroscopically measured contact angle does not necessarily correspond to the microscopic angle of the interface with the substrate. In such a case, the contact line may be pinned to a microscopic ridge, so that effectively one has to introduce an advancing contact angle $\theta_{a}>\theta$ and a receding contact angle $\theta_{r}<\theta$ (these are the macroscopic angles one observes when the contact line advances or recedes along the substrate), or even a velocity-dependent dynamic contact angle. While cyclically changing the volume of a droplet, the macroscopic contact angle will then depend on the history of the process. This is called contact angle hysteresis.

We will neglect the effects of contact angle hysteresis and dynamic contact angles in this work, assuming our surfaces to be ideally smooth. 


\subsubsection{Capillary Condensation}

When a porous solid is embedded in liquid vapor, it can be observed that liquid spontaneously condenses into pores. The reason for this is that the curved meniscus of a liquid in a small pore, and the associated large negative Laplace pressure, allow the liquid to condense even below the saturation vapor pressure. This has been expressed by Lord Kelvin in what is known as the Kelvin equation, relating the mean curvature $H$ to the supersaturation $p_{v} / p_{\text {sat }}$ of the vapor:

$$
\ln \left(\frac{p_{v}}{p_{\text {sat }}}\right)=\frac{\Delta p}{\left(\rho_{l}-\rho_{v}\right) k_{B} T}
$$

(see, for example, [Isr91]). This assumes that the Laplace pressure $\Delta p$ is much larger than the pressure difference $p_{v}-p_{\text {sat }}$, i.e., it only holds for large curvatures, and therefore for small liquid droplets.

A consequence of this is that in a system of several droplets, larger droplets (with a lower vapor pressure) will tend to grow by condensation, while droplets that are so small that their vapor pressure exceeds that of the system evaporate. This process is known as Ostwald ripening.

\subsubsection{Capillary Forces}

When a liquid condenses between two solid bodies, the force exerted by the interfacial stresses along the contact line and the force arising from the pressure difference between liquid and vapor add up to a capillary interaction force between the two bodies. This can manifest either in an attractive or repulsive force, or in torques between tilted objects.

To avoid the appearance of phase boundaries, and therefore of stresses induced by surface tension terms, evaporation of the solvent to create an aerogel needs to be done with careful control of pressure and temperature in order to avoid the region of nucleation. This process is called supercritical drying, since it circumnavigates the critical point in the $p$ - $T$-phase diagram, and is very laborious.

A straight-forward way to calculate forces and torques caused by capillary action is to determine the equilibrium value of the interfacial free energy for a given solid geometry, and then calculate the derivative of this energy with respect to geometrical parameters. The derivative with respect to the distance between the bodies gives the force, while the derivative with respect to the 
angle between the two bodies' orientations gives the capillary torque. The latter is what interests us in the context of wet fiber networks.

\subsubsection{Determining Liquid Morphologies}

Coming back to the three-phase scenario of a liquid on a solid substrate (see Fig. 2.3), we want to minimize the free energy of the liquid with respect to the shape of the interface in order to determine its equilibrium morphology. We call the free interface of the droplet with the vapor phase $\mathcal{S}$, and the interface on the substrate $\mathcal{S}^{*}$. Since $\mathrm{d} A_{s v}=-\mathrm{d} A_{s l}$, the free energy functional of the liquid interfaces is then

$$
\begin{aligned}
\mathcal{F} & =\sigma_{l v} \int_{\mathcal{S}} \mathrm{d} A_{l v}+\left(\sigma_{s l}-\sigma_{s v}\right) \int_{\mathcal{S}^{*}} \mathrm{~d} A_{s l} \\
& =\sigma_{l v}\left(\int_{\mathcal{S}} \mathrm{d} A_{l v}-\cos \theta \int_{\mathcal{S}^{*}} \mathrm{~d} A_{s l}\right) .
\end{aligned}
$$

This has to be minimized in order to determine the shape of the liquid interface in a given solid geometry. It is always possible that several shapes with different topologies that form a local minimum of 2.10 coexist in a given solid geometry. Conversion between these shapes can be associated with an energy barrier that needs to be overcome in the course of the necessary deformations of the interface. Thus, capillary systems can show considerable hysteresis. It is this kind of hysteresis that, on the microscopic length scale, leads to contact angle hysteresis when microscopic roughness is present.

Laplace's condition of constant mean curvature of the free liquid interface, together with Young's contact angle and the condition of either fixed Laplace pressure of fixed liquid volume allow us to explicitly construct an equilibrium liquid morphology between two cylinders that are very lightly tilted, as will be shown in section 2.2. Alternatively, numerical minimization of 2.10 allows us to calculate these shapes for arbitrary tilt, as will be shown in section 2.3 .

\subsection{Analytical Description of Wetting Between Cylinders}

The specific wetting geometry of two parallel cylinders of equal radius has been addressed first by H. M. Princen [Pri69a]. Assuming the existence of 


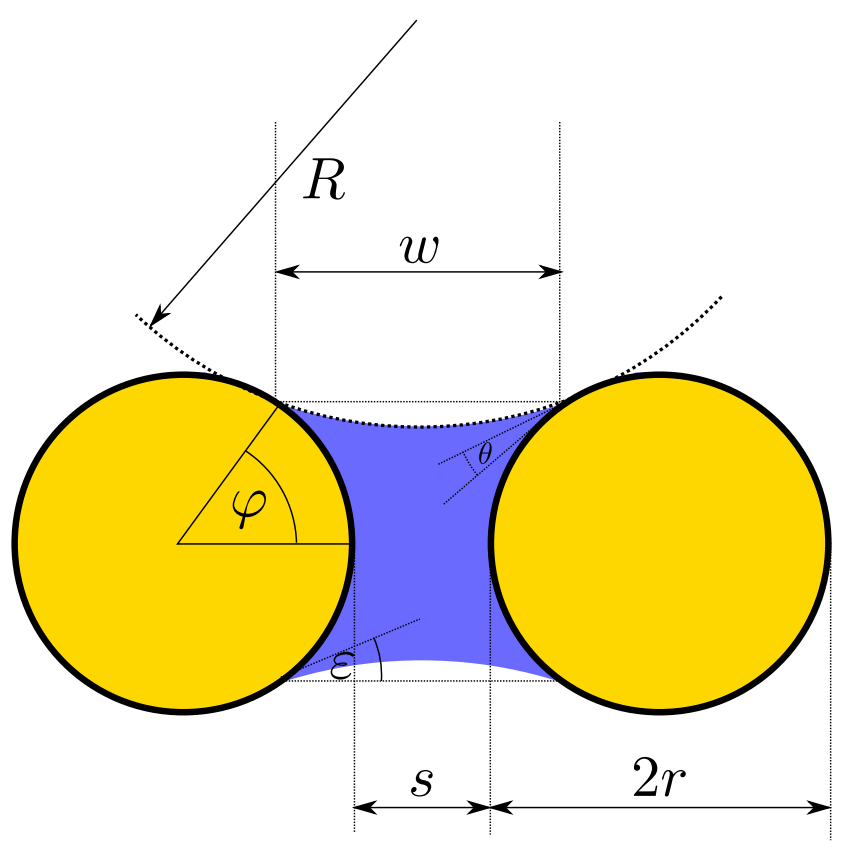

Figure 2.4: Cut through a liquid filament between two cylinders, far away from the meniscus

equilibrium shapes with a nearly translationally invariant cross section he was able to predict the Laplace pressure and cross section of the liquid for a given separation distance and Young's contact angle. We will call this important state a "filament" of the wetting liquid. In the following, we will give an analytic description of this state. We will then show that it can be generalized to the case of slightly tilted cylinders.

The most important feature of a liquid filament is its translational invariance over a large length of the fibers. When we assume that the liquid spreads over a length $L$ along the fiber interstice that is very large compared to the separation $s$ of the fibers, then it is justified to assume that the cross section of the liquid will be the same everywhere except very close to the saddleshaped terminal menisci at the very ends of the filament. Consequently, the radius of curvature along the stretch of the filament is essentially infinite in this approximation. Since the liquid interface is a surface of constant mean curvature, it follows that in any cross section of the filament between parallel cylinders, the interface is a circular arc of fixed radius $R$.

Figure 2.4 gives a sketch of this cross section. It also depicts the following geometric quantities that will be used from here on: The separation of the 
cylinders is called $s$, the cylinders' radius is $r$, the radius of curvature of the liquid filament in the cross sectional plane is denoted by $R$, the contact angle of the liquid is $\theta$, the angle $\varphi$ determines the position of the three-phase contact line, $w$ gives the separation of the contact lines between both cylinders and $\varepsilon=\varphi+\theta-\frac{\pi}{2}$ is an auxiliary angle.

For convenience, we will rescale all lengths by the radius $r$ of the cylinder. Consequently, all areas and volumes will be measured in units of $r^{2}$ and $r^{3}$, respectively. Energies and torques are non-dimensionalized by the energy scale $\sigma_{l v} r^{2}$, while the Laplace pressure is expressed in units of $\sigma_{l v} / r$. The distance $w$ of the contact lines can be expressed by the cylinder separation as follows:

$$
w=s+2(1-\cos \varphi) .
$$

The radius of curvature of the free interface is then simply

$$
R=\frac{w}{2 \sin \varepsilon}=\frac{s+2(1-\cos \varphi)}{2 \sin \varepsilon} .
$$

The shapes of the filament are therefore parametrized by a single free independent parameter, e.g. $\varphi$, once the separation and radius of the cylinders and the liquid contact angle $\theta$ are prescribed.

In order to calculate the interfacial free energy and liquid volume, it is useful to know the contour length of the interfaces in the cross section, as well as the cross sectional area of the liquid:

The contour lengths of the liquid-vapor interface and the liquid-solid interface, $L_{l v}$ and $L_{l s}$ are simply given by:

$$
\begin{aligned}
& L_{l v}=4 R \varepsilon \\
& L_{l s}=4 \varphi,
\end{aligned}
$$

while the cross sectional area $A$ takes the form

$$
A=\frac{2(\varepsilon-\sin \varepsilon \cos \varepsilon) R^{2}}{\sin ^{2} \varepsilon}+4 \sin \varepsilon \sin \varphi R-\frac{2(\varphi-\sin \varphi \cos \varphi)}{\sin ^{2} \varphi} .
$$

The shape parameter $\varphi$ has a lower bound $\varphi_{<}$that determines the point where the two menisci touch. Below this point, no filament can be constructed. For hydrophobic contact angles $(\theta>\pi / 2)$ this is simply $\varphi_{<}=0$. For hydrophilic contact angles, the limit is determined by

$$
\begin{aligned}
\sin \varphi_{<} & =R(1-\cos \varepsilon) \\
& =\left(s+2\left(1-\cos \varphi_{<}\right)\right)\left(1-\sin \left(\theta+\varphi_{<}\right)\right) .
\end{aligned}
$$


The maximum angle is given by the point where the liquid completely engulfs the cylinders. It is

$$
\varphi_{>}=\left\{\begin{array}{ll}
\pi & \text { for } \theta<\frac{\pi}{2} \\
\frac{3 \pi}{2}-\theta & \text { for } \theta \geq \frac{\pi}{2}
\end{array} .\right.
$$

Now consider the total interfacial free energy of such a translationally invariant liquid filament with a fixed Volume $V=L A$. It reads:

$$
F=\left(L_{l v}-L_{l s} \cos \theta\right) L .
$$

When the separation $s$ and liquid volume $V$ are given, the filament length $L=V / A$ and the contour lengths $L_{l v}$ and $L_{l s}$ are functions of $\varphi$ only, according to equations 2.14 and 2.13.

With this, the value $\varphi$ will take on in mechanical equilibrium can be determined by finding the minimum of $F$ with respect to $\varphi$.

\subsubsection{Condition of mechanical equilibrium}

Employing the interfacial free energy per unit length of the filament $f=F / L$, the condition of stationarity with respect to changes in the wetted length is then simply given by the condition

$$
\partial_{\varphi} F=\frac{\left(A \partial_{\varphi} f+f \partial_{\varphi} A\right) V}{A^{2}}=0
$$

or equivalently

$$
\frac{f}{A}=\frac{\partial_{\varphi} f}{\partial_{\varphi} A}=\partial_{A} f=0
$$

This latter condition can be interpreted in a geometrical way: The tangent to a point of the graph of the function $F(A)$ has to pass through the origin $f=0$ and $A=0$ of the coordinate system. In general, one can show that

$$
\partial_{A} F=\Delta p=2 \sigma_{l v} H
$$

The relation above can be checked for the particular form of $F$ and $A$ by explicit calculation. Since we assumed that the free interface in a cross section follows a circular arc of radius $R$, and is translationally invariant along the cylinder axis, the mean curvature $H$ equals $1 / 2 R$. Equation 2.19 then assumes the explicit form

$$
R^{2}(\varepsilon+\cos \varepsilon \sin \varepsilon)-2 R(\varphi \cos \theta+\sin \varepsilon \sin \varphi)+(\varphi-\sin \varphi \cos \varphi)=0 .
$$


Solving this equation leads to the radius of curvature $R(\theta, \varphi)$ in equilibrium as a function of the prescribed contact angle $\theta$ and the parameter $\varphi$. One can then calculate the separation $s(\theta, \varphi)$ from equation $(2.12)$. This function reaches a maximum $s^{*}$ at a parameter value $\varphi_{\max }$, meaning that the values of separation distances $s$ where locally stable liquid filaments are found is limited by $s^{*}$, which depends on the contact angle $\theta$.

The corresponding Laplace pressure $\Delta p$ of an equilibrated filament turns out to be a monotonously increasing function of the distance $s$ up to $s^{*}$, where the filament becomes unstable with respect to a longitudinal redistribution of liquid. Hence, there is also a maximum Laplace pressure $\Delta p^{*}$ which cannot be surpassed by a liquid filament. This maximum pressure is a function of the contact angle $\theta$.

\subsubsection{Modified Princen Model}

In this section we will expand the Princen model for parallel cylinders to the case of small finite crossing angles $\alpha \rightarrow 0$ to extract the scaling of the total liquid volume $V$ of a filament at fixed Laplace pressure $\Delta p$. In a similar fashion, one can compute the interfacial energy $\mathcal{F}$, torque $T$, and length $L$ of the liquid filament. To this end, we will evoke the theory of Princen outlined in the previous section and modify it to account for the modified local geometry of tilted cylinders. While the prefactors of the scaling laws for the volume and torque can only be determined numerically in the generic case of finite Laplace pressure, an expression valid for asymptotically small Laplace pressures $\Delta p \rightarrow$ $-\infty$ can be found by purely analytical arguments. Throughout the following analysis we will assume a zero separation distance $s=0$ between the surfaces of the cylinders, i.e., the two cylinders touch in a single point. The separation of two cylinder cross sections is then simply given by the angle $\alpha$ and the distance from the crossing point (see figure 2.5).

We will start with a number of general observations. A simple scaling relation between the volume of the liquid for a given fixed Laplace pressure at small crossing angles $\alpha \ll 1$ can be obtained from purely geometrical reasoning: if we scale the dimensions of the two wetted cylinders and the liquid filament into the direction of the bisecting line (thus decreasing $\alpha$ in a linear approximation), the liquid filament will stay close to a family of new equilibrium configurations. Because the curvature of the liquid interface along the cylinders can be neglected, the local mean curvature and thus the Laplace pressure will remain unchanged compared to the unstretched configuration. 


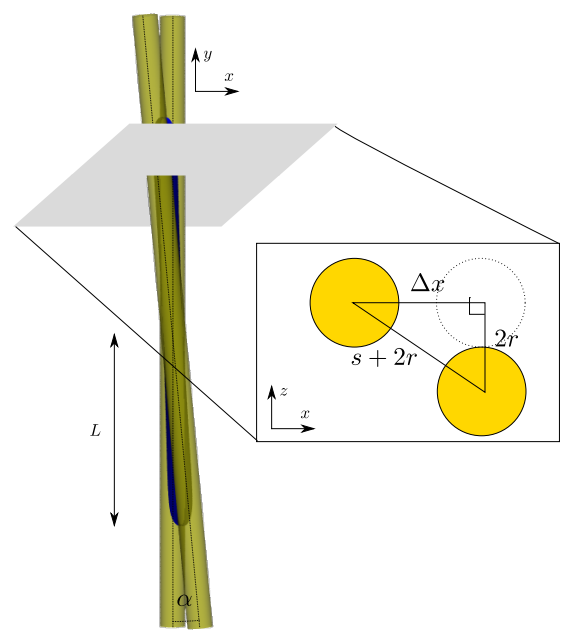

Figure 2.5: Two tilted cylinders with liquid filament; the cross section shows the distances involved

This implies that the volume $V$, length $L$ and interfacial free energy $\mathcal{F}$ will scale with $\alpha^{-1}$. This will be confirmed in the following.

In the theory of Princen, the Laplace pressure $\Delta p$ of a liquid filament of given volume in equilibrium is a function of the separation $s$. We can therefore expect that when a certain pressure $\Delta p$ is prescribed in a configuration of almost parallel cylinders, a liquid filament will spread to a point where the surfaces are separated by a certain distance $s_{f}$. The value of $s_{f}$ will depend on the prescribed Laplace pressure $\Delta p<\Delta p^{*}$ and material contact angle $\theta$, but not on the crossing angle $\alpha$. Since the condition that determines the value of $s_{f}$ is unaffected by a dilation of the liquid shape and cylinders along the $y$-direction, we can expect the asymptotic scaling $L \propto \alpha^{-1}$ for the distance of the terminal menisci from the contact point and $V \propto \alpha^{-1}$ for the total liquid volume of the filament in the regime of $\alpha \ll 1$. Similar arguments show that the torque will scale as $\alpha^{-2}$ for a fixed Laplace pressure.

The length $L$ of the filament at given Laplace pressure can be calculated from the separation $s_{f}$ (see figure 2.5):

$$
L=2 \sqrt{s_{f}\left(4+s_{f}\right)} \alpha^{-1} \text {. }
$$

The separation $s_{f}$ corresponds to a mechanically equilibrated filament at a Laplace pressure $\Delta p<\Delta p^{*}$ as determined from equations (2.21) and (2.12) for a radius $R=\Delta p^{-1}$. 
The volume $V$ of the liquid filament can be computed from the integral over the cross sectional area $A$ perpendicular to the bisecting line:

$$
V=2 \int_{0}^{L} \mathrm{~d} y A(y) .
$$

Here, $y$ denotes the distance of the cutting plane and $L$ the distance of the terminal meniscus from the kissing point of the cylinders. As we assume the distortion of the filament due to the tilt of the cylinders to be small, the cross section of the filament will depend only on the separation distance $s$, which itself varies with the lateral position $0 \leq y \leq L$ on the bisecting line. By applying the theorem of Pythagoras we find, again,

$$
y=\alpha^{-1} \sqrt{(s+4) s}
$$

(see also figure 2.5). For a given Laplace pressure $\Delta p$ of the filament the radius of the meniscus is fixed to $R=\Delta p^{-1}$. This allows us to express the area $A$ of the filament's cross section and the corresponding separation distance $s$ by the local opening angle $\varphi$ of the filament and the auxiliary angle $\varepsilon$.

Solving the equilibrium condition equation 2.21 for the radius $R$ yields the Laplace pressure as a function $\Delta p_{f}\left(\varphi_{f}, \theta\right)$ of the corresponding opening angle $\varphi_{f}$ of the filament close to the terminal meniscus and the material contact angle $\theta$. Then the maximal opening angle $\varphi_{\max }$, which is attained close to the terminal meniscus, is determined by the condition $\Delta p=\Delta p_{f}\left(\varphi_{\max }, \theta\right)$ for a given Laplace pressure $\Delta p$ and contact angle $\theta$. The minimal opening angle $\varphi_{\min }$ of the filament, reached at the contact point, is determined from equation 2.12 with the condition $s(\Delta p, \theta, \varphi)=0$ for a given Laplace pressure $\Delta p$.

Making use of the chain rule of differentiation we are now ready to rewrite integral equation 2.23 in the form

$$
\begin{aligned}
& V(\alpha, \Delta p, \theta)= \\
& \quad 2 \int_{\varphi_{\min }}^{\varphi_{\max }} \mathrm{d} \varphi A(\Delta p, \theta, \varphi) \frac{\partial s}{\partial \varphi}(\Delta p, \theta, \varphi) \frac{\partial y}{\partial s}(\alpha, s(\Delta p, \theta, \varphi)) .
\end{aligned}
$$

It is evident from the derivative

$$
\frac{\partial y}{\partial s}=\frac{2+s}{\alpha \sqrt{s(s+4)}}
$$

of the function $y(s)$ in equation (2.24) that the total volume satisfies the scaling form $V(\alpha, \Delta p, \theta)=\alpha^{-1} V(1, \Delta p, \theta)$. The latter integral $V(1, \Delta p, \theta)$ 
can be computed numerically for given Laplace pressure $\Delta p$ and material contact angle $\theta$.

In a similar fashion we can compute the torque $T$ between the two cylinders for a fixed Laplace pressure $\Delta p$. The forces per length, $k$, between the two cylinders are composed of the surface tension forces of the liquid interfaces and the force of the Laplace pressure; they can be expressed by the force per unit length

$$
k=2(\cos \varepsilon-\Delta p \sin \varphi) .
$$

With this we are able to write the torque $T$ in form of an integral

$$
T=2 \int_{0}^{L} \mathrm{~d} y g(\alpha, y) k(y) y
$$

with a function

$$
g(\alpha, y)=\frac{(4+s) s}{\alpha(2+s)}
$$

that accounts for the relevant component of the force $k$. This integral can be transformed into an integral similar to 2.26 and solved numerically for different Laplace pressures and contact angles. From the form of the integral the scaling $T(\alpha, \Delta p, \theta)=\alpha^{-2} T(1, \Delta p, \theta)$ is evident.

\subsubsection{Analytical model for $\Delta p \rightarrow-\infty$}

In this subsection we will compute the volume $V$, interfacial energy $\mathcal{F}$ and extension $L$ of the liquid filament for asymptotically small Laplace pressures $\Delta p \rightarrow-\infty$. In contrast to the modified Princen model in the previous subsection, this model is valid for arbitrary crossing angles $\alpha$. The fundamental idea behind the following calculation is a separation of length scales. In the limit of large negative Laplace pressures, the wetting liquid will always be located around the kissing point of the two cylindrical surfaces (see figure 2.6). In this limit, the radius of curvature of the meniscus perpendicular to the tangent plane of the cylindrical surfaces becomes small when compared to the remaining two dimensions of the filament, and the two cylinders are described by gently sloping surfaces close to the contact point. The height $H_{m}$ of the meniscus is effectively fixed by the Laplace pressure $\Delta p$ and the contact angle $\theta$. This assumption is justified as long as the filament is sufficiently flat, i.e., the curvature of the meniscus in the tangent plane to the contact point is 

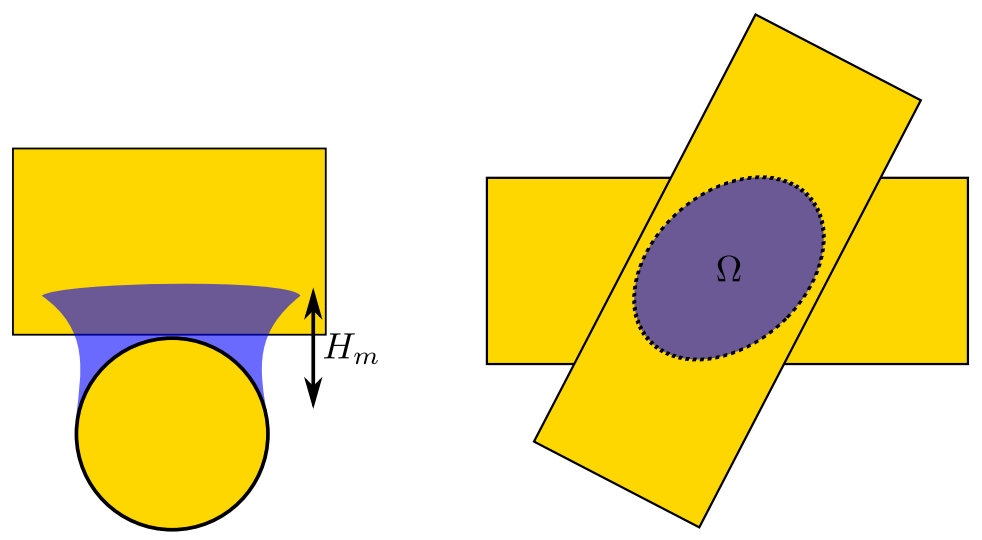

Figure 2.6: Illustration of the shape of the meniscus in the limit of large negative Laplace pressures. The left images shows a side view, while in the right image the cylinders are seen from the top with the projected shape of the liquid between them sketched in as the blue area.

much smaller than the out of plane curvature of the contour. Without loss of generality, we place our Cartesian coordinate system such that the tangent plane is identical to the $x y$-plane.

Here, and in what follows, we make use of the dimensionless rescaled height $h=H_{m} / R$; the corresponding Laplace pressure reads

$$
\Delta p=\frac{2 \cos \theta}{h} \text {. }
$$

The in-plane contour of the filament is now given by the implicit condition of fixed height $\Delta s(x, y)=h(\Delta p, \theta)$, where $\Delta s$ is the width of the gap between the two cylindrical surfaces close to their contact point. The solution to this problem is a two-dimensional space curve $(x(t), y(t))$, where $t$ is a dimensionless parameter. Placing the kissing point of the cylinders in the coordinate origin, the gap width $\Delta s$ at a point $(x, y)$ can be approximated by

$$
\Delta s=\frac{1}{2}\left[\sin ^{2} \alpha x^{2}+\left(1+\cos ^{2} \alpha\right) y^{2}+2 \sin \alpha \cos \alpha x y\right] .
$$

The condition $\Delta s=h$ can now be written as

$$
(x, y) \cdot \mathbf{M}(x, y)^{T}=2 h
$$

with a matrix

$$
\mathbf{M}=\left(\begin{array}{cc}
\sin ^{2} \alpha & \sin \alpha \cos \alpha \\
\sin \alpha \cos \alpha & 1+\cos ^{2} \alpha
\end{array}\right) .
$$


More generally, equation 2.32 defines a set of isolines of given distance $h$ between two tilted cylindrical surfaces close to the contact point.

From the elongated shape of the filaments we expect an ellipse for the contour. This is in accord with the quadratic form (2.32). The lengths of the principal axes $a$ and $b$ of this ellipse are then related the zeros in $\lambda$ of

$$
|\mathbf{M}-\lambda \mathbf{1}|=0 .
$$

Explicit computation of the characteristic polynomial (2.34) yields

$$
\lambda_{1}=1-\cos \alpha \quad \text { and } \quad \lambda_{2}=1+\cos \alpha .
$$

Close to the contact point of the surfaces, any isoline of equal distance $h$ between two tilted cylinders can be approximated by an ellipse with long and short axis

$$
a=\sqrt{\frac{2 h}{1-\cos \alpha}} \text { and } b=\sqrt{\frac{2 h}{1+\cos \alpha}},
$$

respectively.

Based on this observation, we can calculate the volume $V$ of a small liquid filament from the two dimensional integral

$$
V=\int_{\Omega} \mathrm{d} S \Delta s(x, y)
$$

over the domain $\Omega$, which is bounded by the contour line of the meniscus. For the case of $\alpha=\pi / 2$, we find $a=b=\sqrt{2 h}$. In this case, the two dimensional domain $\Omega$ is a circle of radius

$$
R_{\mathrm{f}}=2 \sqrt{-\frac{\cos \theta}{\Delta p}}
$$

where we used equation 2.30 to express $h$ by the Laplace pressure $\Delta p$ and the contact angle $\theta$. In polar coordinates $\phi=\arctan (x / y)$ and $\rho=\sqrt{x^{2}+y^{2}}$ the gap width is $\Delta s=\rho^{2} / 2$ and the volume of the circular liquid bridge reads

$$
V_{l}=2 \pi \int_{0}^{R_{\mathrm{f}}} \mathrm{d} \rho \rho \Delta s=\frac{4 \pi \cos ^{2} \theta}{\Delta p^{2}} .
$$

For the general case of $\alpha \neq \pi / 2$, we find by a simple affine transformation in the tangent plane that the liquid volume of an oblique liquid filament is

$$
V_{l}=\frac{4 \pi \cos ^{2} \theta}{|\sin \alpha| \Delta p^{2}} \approx 4 \pi \cos ^{2} \theta|\alpha|^{-1} \Delta p^{-2} .
$$


The length $L$ of such an elliptical filament assumes the form

$$
L=2 a=4 \sqrt{-\frac{\cos \theta}{(1-\cos \alpha) \Delta p}} \approx 4 \sqrt{2 \cos \theta}|\alpha|^{-1}(-\Delta p)^{-1 / 2},
$$

while the area $A_{l s}$ of the cylinders wetted by the liquid is given by

$$
A_{l s}=2 \pi a b=-\frac{8 \pi \cos \theta}{|\sin \alpha| \Delta p} \approx-8 \pi \cos \theta|\alpha|^{-1} \Delta p^{-1} .
$$

In the limit of flat filaments one may safely neglect the contribution of the free liquid interface. Then, the grand canonical interfacial energy $\mathcal{F}^{\prime}=\mathcal{F}-\Delta p V_{l}$ for a fixed Laplace pressure assumes the form

$$
\mathcal{F}^{\prime}=-\frac{4 \pi \cos ^{2} \theta}{|\sin \alpha| \Delta p}
$$

The corresponding capillary torque $T$ is given by the derivative

$$
T=\frac{\partial \mathcal{F}^{\prime}}{\partial \alpha}=-\frac{4 \pi \cos ^{2} \theta \cos \alpha}{\left|\sin ^{2} \alpha\right| \Delta p} \approx-4 \pi \cos ^{2} \theta \alpha^{-2} \Delta p^{-1}
$$

The scaling of the above quantities for the case of a constant liquid volume $V$ can be obtained by solving equation 2.40 for the Laplace pressure:

$$
\Delta p \approx-(2 \pi)^{1 / 2} \cos \theta V_{l}^{-1 / 2}|\alpha|^{-1 / 2}
$$

Inserting the expression 2.45 into expressions 2.41 for the length and 2.44 for the torque yields the scaling relations

$$
L \approx 4(2 / \pi)^{1 / 4} V_{l}^{1 / 2}|\alpha|^{-3 / 4}
$$

for the total length and

$$
T \approx 2(2 \pi)^{1 / 2} \cos \theta V_{l}^{1 / 2}|\alpha|^{-3 / 2}
$$

for the capillary torque.

Now, we are ready to compare the prefactors of the scaling forms for the torque obtained in the modified Princen model to the corresponding prefactors for the elliptical filaments valid for small Laplace pressures $\Delta p \rightarrow-\infty$. Figure 2.7 shows the prefactor $T_{0}$ of the torque for different fixed Laplace pressures in the modified Princen model, arrived at by numerical integration of (2.28), and multiplied with $\Delta p$ in accordance with the expected scaling from (2.44). The solid line shows equation (2.44). The curves do indeed converge for very large negative Laplace pressures.

In the next section, we will verify the scaling behavior of the torque for arbitrary Laplace pressures and crossing angles using numerical minimizations of the interfacial free energy to obtain exact shapes for the liquid interface. 


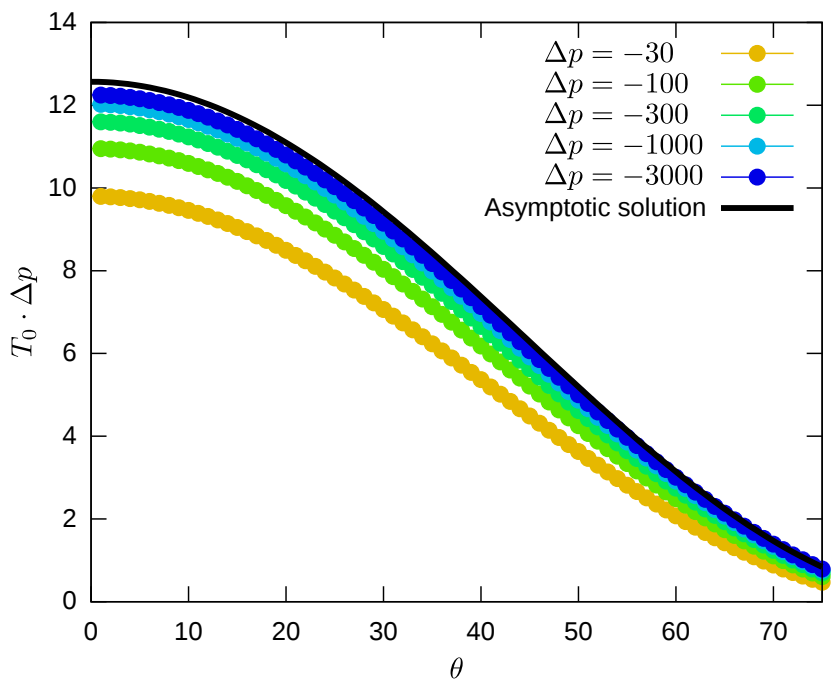

Figure 2.7: Coefficient $T_{0}$ of the capillary torque as a function of contact angle $\theta$, rescaled by the Laplace pressure. The colored circles are calculated from the modified Princen model, while the solid black line corresponds to the asymptotic solution for large negative Laplace pressures.

\subsection{Numerical Computation}

In order to find minimal surfaces in nontrivial geometries, numerical minimization techniques can be a valuable tool. We used the Surface Evolver package by Ken Brakke [Bra92] to gain exact numerical solutions for the problem of wetting between cylinders at finite crossing angles $\alpha$, without having to resort to small-angle approximations as we did in section 2.2.

Similar calculations have been done by Bedarkar and Wu [BW09], and by Virozub et al. [VHB09], albeit in a limited range of parameters, using only very little data points. Also, the surface evolver approach used in BW09] explicitly includes the liquid-solid interface, which makes it prone to numerical instabilities. Consequently, the results we show in the following differ considerably.

Our primary aim is to characterize the dependence of the interfacial free energy on the cylinder crossing angle $\alpha$, so that we may derive the capillary torques acting between crossing cylinders, over the full range of values for $\alpha$, and for different parameters such as contact angle $\theta$, fixed volume $V$ or fixed Laplace pressure $\Delta p$. 

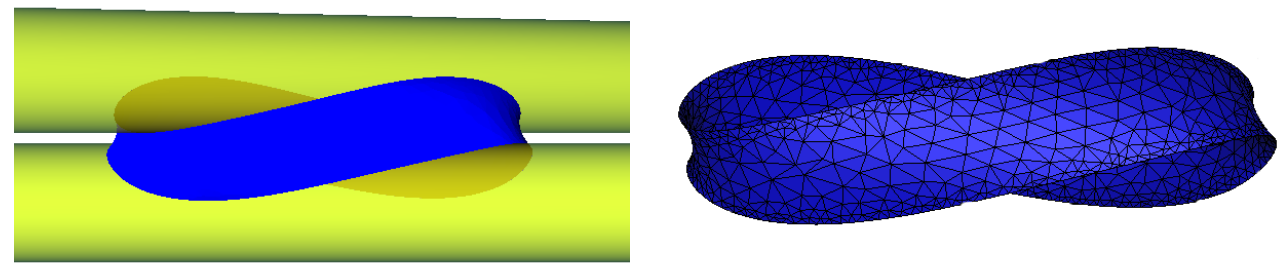

Figure 2.8: Surface Evolver simulation of a liquid filament between two tilted cylinders at $\alpha=30^{\circ}$. In the left image, the cylinders are made slightly transparent. The right image shows the triangularization of the liquid interface.

The method is to create a triangular mesh approximating the liquid-vapor interface, which is topologically equivalent to the liquid morphology one wants to study. The nodes of the mesh are then moved according to a conjugate gradient scheme, while observing constraints to keep the nodes of the contact line tethered to the substrate, and further optional constraints like that of constant volume. The surface evolver offers a variety of tools to fine-tune the triangularization of the surface and the minimization procedure. We chose a fine triangularization close to the contact line, with larger mesh elements further away (see Fig. 2.8).

The surface evolver minimizes equation (2.6), treating $\Delta p$ either as a Lagrange multiplier to satisfy a fixed volume constraint, or as a fixed Laplace pressure. It is advantageous for numerical speed and stability to only model the liquid-vapor interface. The surface integrals in (2.10) can then be expressed as line integrals over the three-phase contact line via Stokes' theorem. The same is done for the integral to calculate the volume in (2.6). The surface evolver calculates these line integrals by summation over the vertices of the three-phase contact line.

The geometrical problem is again defined as that of two cylinders touching at at the coordinate origin, and tilted against each other by an angle $\alpha$. Once 


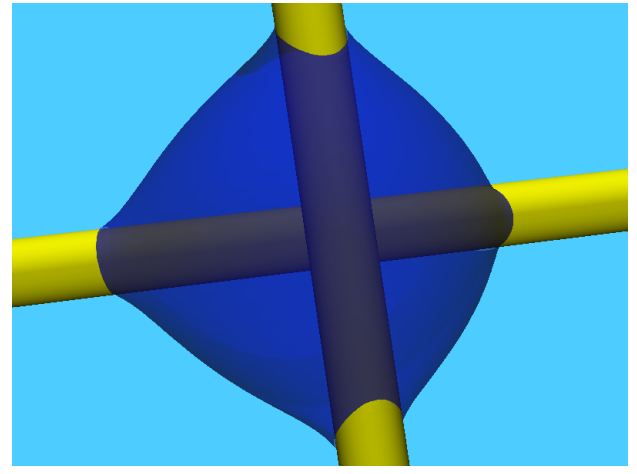

(a) droplet state

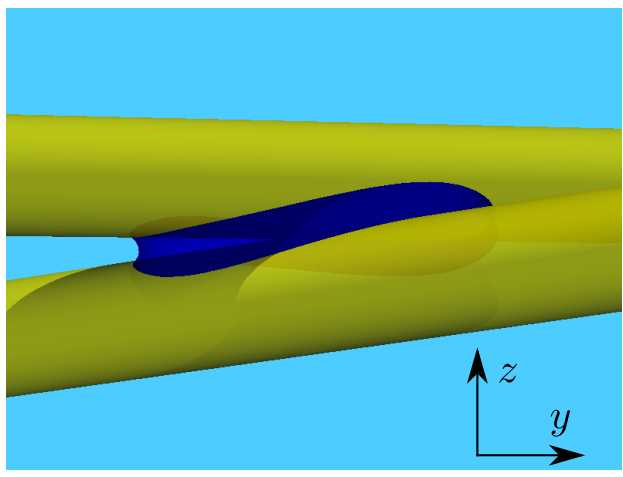

(b) filament state

Figure 2.9: The two main liquid morphologies found between cylindrical fibers in contact

more, we chose the cylinder radius as the basic length scale of the system, and measure energies in terms of the interfacial energy of a liquid-vapor interface of unit area. Further parameters of the simulation are either the prescribed volume $V$ or the Laplace pressure $\Delta p$, the contact angle $\theta$ and the crossing angle $\alpha$.

In the chosen units, the first integral of equation 2.10) is simply the interfacial area, calculated by summation of the triangle areas of the mesh. The second integral is transformed into a line integral over the three-phase contact line as follows:

$$
\begin{aligned}
\int_{\mathcal{S}^{*}} \mathrm{~d} A_{s l} & =\int_{\mathcal{S}^{*}} \mathbf{n} \cdot \mathrm{d} \mathbf{A}_{s l}=\int_{\partial \mathcal{S}^{*}} \mathbf{w} \cdot \mathrm{d} \mathbf{l} . \\
\nabla \times \mathbf{w} & =\mathbf{n} .
\end{aligned}
$$

Here, $\mathbf{n}$ is a unit vector normal to the liquid-vapor interface, $\mathrm{d} \mathbf{A}_{s l}$ is the vectorial area element, also pointing normal to the interface, $\mathrm{d} \mathbf{l}$ is a line element of the three-phase contact line, and the vector $\mathbf{w}$ needs to be chosen such that it fulfills 2.49). Then, the last equality in equation 2.48 follows from the law of Stokes.

We chose the first cylinder to be parallel to the $y$-axis, and a distance $d / 2$ above the origin in $z$-direction, while the second cylinder is $d / 2$ below the 
origin and forms an angle $\alpha$ with the $y$-axis. Then we choose

$$
\mathbf{w}_{1}=\frac{1}{r}\left(\begin{array}{c}
0 \\
\left(z-\frac{d}{2}\right) y \\
x y
\end{array}\right) \quad \mathbf{w}_{2}=\frac{1}{r}\left(\begin{array}{c}
\sin \alpha\left(z+\frac{d}{2}\right)(y \cos \alpha+x \sin \alpha) \\
\cos \alpha\left(z+\frac{d}{2}\right)(y \cos \alpha+x \sin \alpha) \\
(x \cos \alpha-y \sin \alpha)(y \cos \alpha+x \sin \alpha)
\end{array}\right)
$$

as vector integrands for the disjunct paths of the three-phase contact line to conform to condition (2.49) for the two cylinder surfaces, respectively.

We employ two different liquid topologies, pictured in figure 2.9. First, a state where the liquid wets the cylinders completely around the contact point, i.e., the liquid is topologically equivalent to a sphere engulfing the cylinder contact point, with four holes where the cylinders cross the interface (see the figure 2.9(a) . The second topology is that investigated in section 2.2, here, the liquid is topologically equivalent to a torus contacting the inner surfaces of the cylinders (see figure 2.9(b)].

The surface evolver is initialized with a simple vertex structure representing the respective topology, and containing a volume that is approximately equal to the value to be investigated. Then, the triangular mesh is refined in a way that emphasizes the three-phase contact line, and the minimization procedure is advanced. These steps are repeated until equilibrium is reached. To judge the convergence of the algorithm, we measure the wetted length $L$ along a cylinder axis, and continue the minimization until the change of $L$ in one step of the algorithm is smaller than $0.5 \%$ of the smallest vertex length of the triangular mesh.

Figure 2.9 shows the relaxed states of the liquid in the two different topologies under investigation. We will call left one $(2.9(\mathrm{a}))$ a droplet state, and the right one $(2.9(\mathrm{~b})]$ a filament state. The droplet state is found to be stable only for large angles $\alpha$ and small volumes, while the filament state becomes unstable when $\alpha$ approaches $\pi / 2$, where it changes into a droplet. However, the filament state is stable over a very wide range of the model parameters. The droplet state shows only a slight deviation from a perfectly spherical droplet, induced by Young's condition on the cylinder surface. Because of this, it also shows little variation with the angle $\alpha$, and is therefore not interesting to us.

To compare the results of the surface evolver with the exact theory as presented by Princen [Pri70] and in section 2.2, we first investigate the case of parallel cylinders with a finite separation $s$. Solving equation (2.21) for the radius of curvature $R$, one arrives at a function $R(\varphi, \theta)$. Together with expression 2.12 for the separation $s(\varphi, \theta)$, this implicitly defines the relationship 


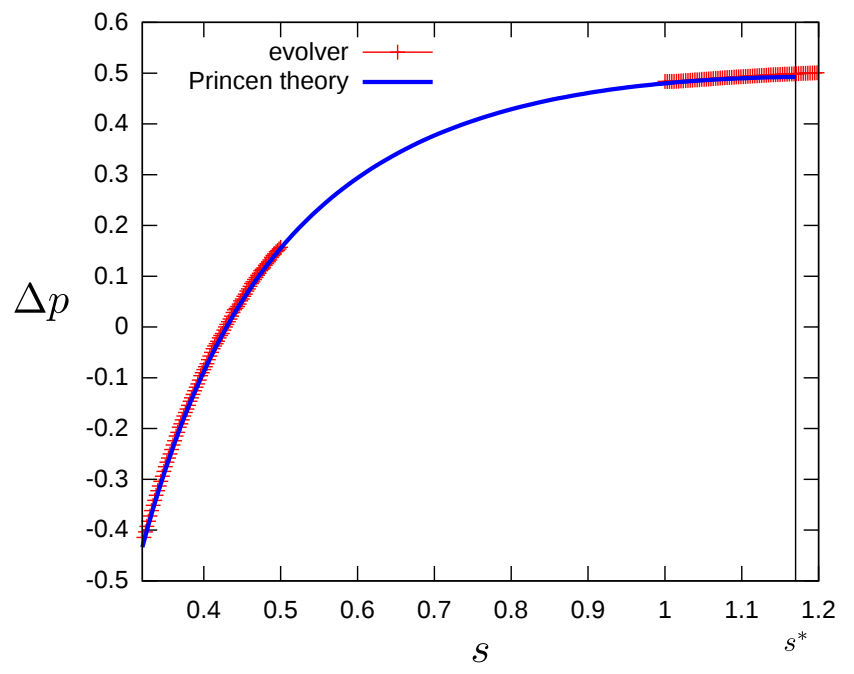

Figure 2.10: Laplace pressure $\Delta p$ vs. separation $s$ of two parallel cylinders

$\Delta p(s)$, giving the Laplace pressure at a given separation. As mentioned in section 2.2, there is a maximum separation $s^{*}$ beyond which no solutions to this equation exist.

Figure 2.10 shows this relationship as the blue line. To compare this to numerics, we initialized the surface evolver with a toroidal liquid topology between parallel cylinders at constant volume and varied the separation $s$. In each step, we waited for convergence of the spreading length $L$, as stated above.

Exploring a wide range of separations $s$ at a given volume in the surface evolver proves difficult, since the same volume of liquid at low separations will spread out very long, and such an elongated structure is difficult to triangularize sensibly. Since the theory is independent of the prescribed volume, though, we ran two simulations, visible as two distinct sets of red crosses in figure 2.10. For both simulations, the agreement with the theoretical curve is very good. It is to be noted that the simulations remained stable for a bit in the regime where no stable solutions of the analytical theory are found.

In the following, we will show the numerical findings for the dependence of the filament state on the angle $\alpha$, while keeping the separation $s=0$ throughout. This scenario of touching cylindrical fibers that can rotate around their crossing point is what we want to model in the context of two-dimensional wet fiber networks later on.

To investigate these angular dependencies, we initialize the surface evolver 


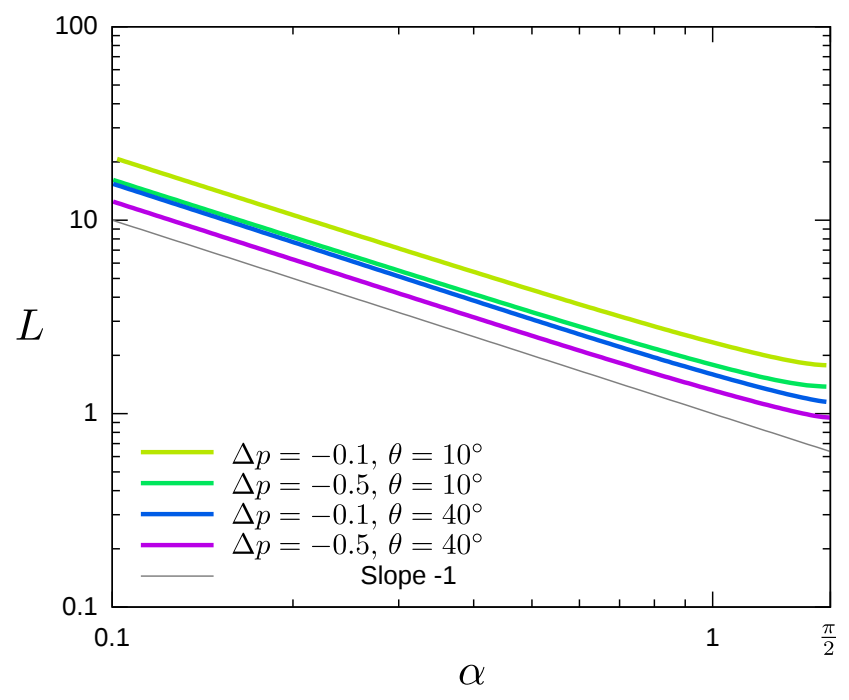

Figure 2.11: Wetted length $L$ as a function of the crossing angle $\alpha$ of two crossing fibers at different equilibrium contact angles $\theta$ and Laplace pressures $\Delta p$.

with a toroidal liquid topology at $\alpha=\pi / 2$ as stated above, and then reduce the angle $\alpha$ to of $98.5 \%$ of its previous value until we reach $\alpha=0.1$, thus taking smaller steps in the regime of small angles, since we expect from section 2.2 that the liquid will spread with a power law in that region. In each step, we adjust the refinement of the mesh and advance the minimization algorithm until the length $L$ converges, as described above. We record the angle $\alpha$, the total interfacial energy $F$, the wetted length $L$ and the liquid volume $V$ or the Laplace pressure $\Delta p$ in every step of this quasi static tilting of the cylinders.

To check the assumption that the spreading length $L$ between tilted cylinders is governed by the separation $s$ at a given Laplace pressure, we plot the wetted length $L$ as function of $\alpha$ in the constant-pressure ensemble (see Fig. 2.11). It can be seen that the small-angle relationship 2.22 holds for a very wide range of angles $\alpha \in(0, \pi / 2)$. The prefactor $\Delta x=\sqrt{s_{f}\left(4+s_{f}\right)}$ gives the lateral distance between the cylinder axes at the point of the terminal meniscus (see figure 2.5).

We plotted the coefficient $\Delta x(\theta, \Delta p)$ from the modified Princen theory, along with the values for $\Delta x$ extracted from a fit of the relation $L=\Delta x \alpha^{-1}$ to the numerical data in figure 2.12 .

While the data are in good qualitative agreement, there is a certain bias to- 


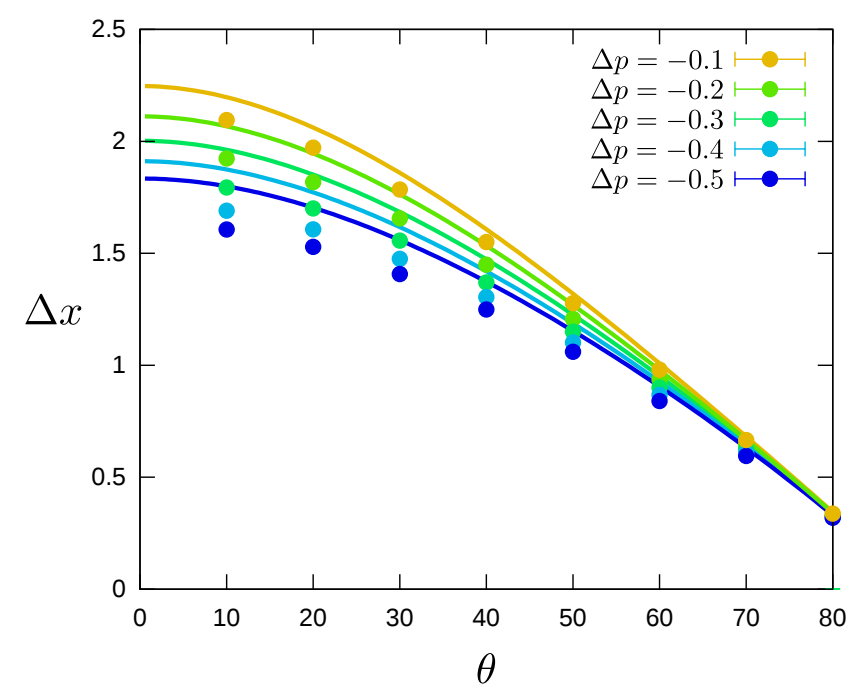

Figure 2.12: Comparison of the coefficient $\Delta x$ of the scaling of the wetted length with $\alpha$ to the separation predicted by the theory for a given Laplace pressure $\Delta p$, as a function of the Young's contact angle $\theta$. Surface evolver results are drawn as circles, while the prediction of the modified Princen model is drawn as lines.

wards lower coefficients, meaning that the assumption that the liquid spreads to the point of separation that corresponds to the prescribed Laplace pressure overestimates the spreading behavior.

To calculate the torque that the filament exerts on the cylinders, we calculate the numerical derivative of the interfacial energy with respect to $\alpha$. Figures 2.13 and 2.14 show the simulation results of the torque as a function of $\alpha$ in the constant pressure and constant volume ensembles, respectively. Again, the scaling behavior predicted at the end of section 2.2 .3 holds over a wide range of angles: In the constant pressure ensemble, the torque varies as $\alpha^{-2}$, while in the constant volume ensemble, it varies as $\alpha^{-3 / 2}$. Only when $\alpha$ approaches $\pi / 2$ can a serious diversion be observed. At that point, the filament state becomes unstable, and one would expect the liquid to transform into a droplet state. Since the droplet state is almost spherical, the torque will therefore vanish at that point.

Figure 2.15 shows the coefficient $T_{0}$ of the power law for the torque, $T=$ $T_{0} \alpha^{-2}$, in the case of constant pressure. It shows that the capillary torque vanishes as $\theta$ approaches $90^{\circ}$, and grows larger for smaller contact angles and 


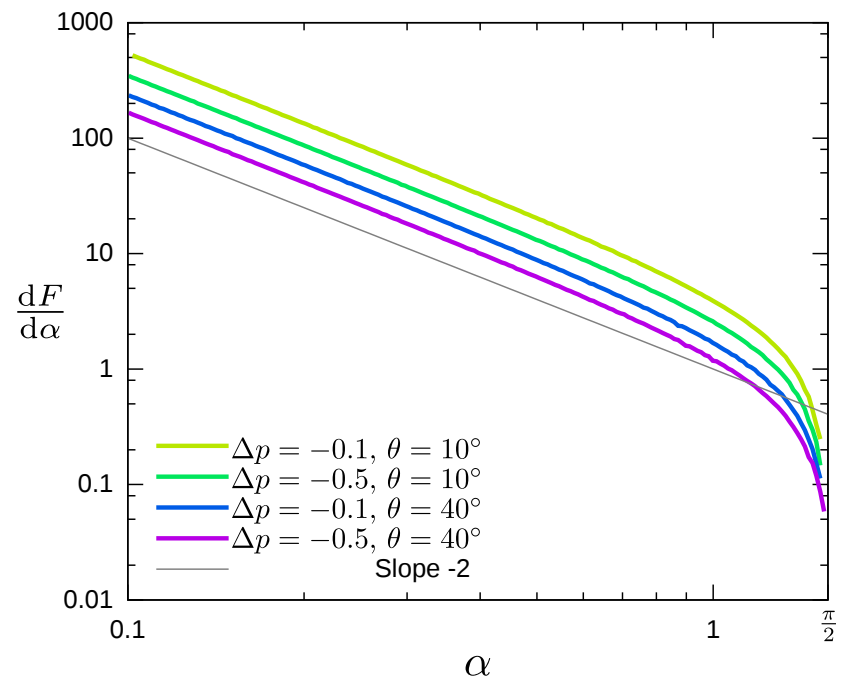

Figure 2.13: Double logarithmic plot of the capillary torque $\mathrm{d} F / \mathrm{d} \alpha$ as a function of the crossing angle $\alpha$ of two crossing fibers at different equilibrium contact angles $\theta$ and Laplace pressures $\Delta p$.

smaller prescribed pressure differences. The solid lines in figure 2.15 are numerical solutions of (2.28). Similarly to the comparison of the spreading length coefficient seen in 2.12 , the theory overestimates the coefficient systematically by a small value.

In the case of constant volume, only small contact angles could be simulated, because at larger contact angles, liquid filaments with a prescribed volume are unstable.

The observed scaling is valid for all contact angles $\theta$, Laplace pressures $\Delta p$ and volumes $V$; these parameters merely influence the coefficient of the scaling law. In order to simulate the mechanical response of a system of cylinders to capillary interactions of filaments that form by capillary condensation at the crossing points, one would therefore have to implement a torque interaction mediated by an angular potential at the crossing points of the form

$$
F(\alpha) \propto \alpha^{\eta}
$$

where the value of $\eta$ depends on the ensemble under consideration. If the vapor pressure of the system is controlled, $\eta=-1$ is the correct choice, while for nonvolatile filaments of equal volume, $\eta=-1 / 2$ needs to be chosen. 


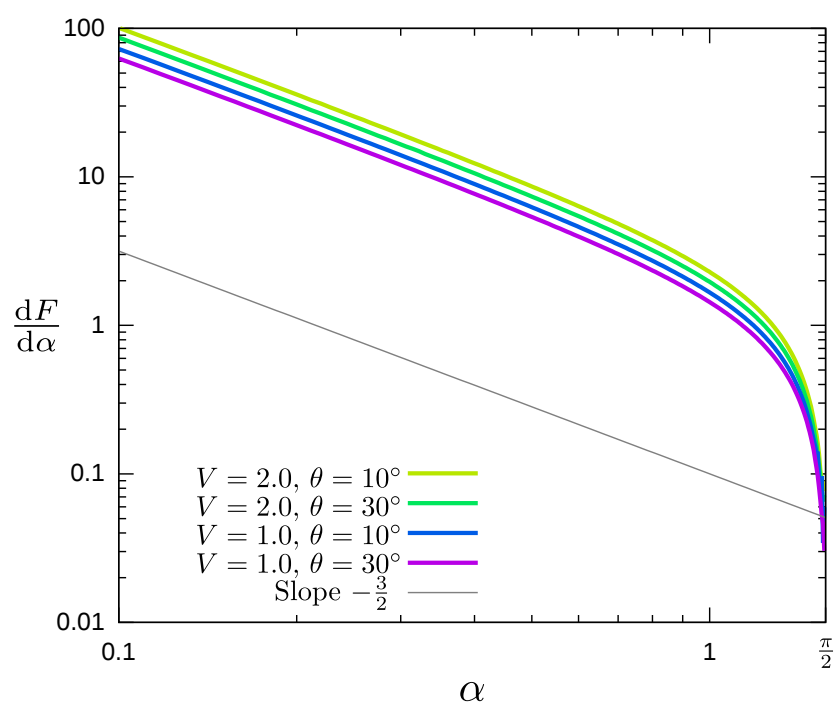

Figure 2.14: Double logarithmic plot of the capillary torque $\mathrm{d} F / \mathrm{d} \alpha$ as a function of the crossing angle $\alpha$ of two crossing fibers at different equilibrium contact angles $\theta$ and liquid volumes $V$.

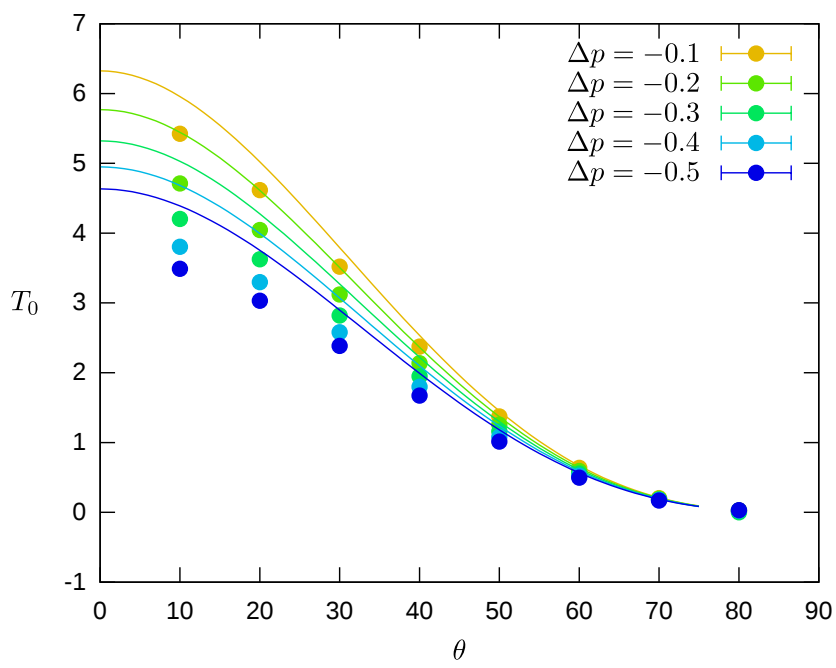

Figure 2.15: Prefactor $T_{0}$ of the power law governing the capillary torque between cylinders in the constant pressure ensemble, as a function of the Young's contact angle $\theta$, for different values of the Laplace pressure $\Delta p$. The circles are results of the surface evolver calculations, while the lines are from the modified Princen model. 


\section{Chapter 3}

\section{Crosslinked Fiber Networks}

In order to describe the effect of liquid bridges when they appear in materials that are composed of large numbers of fibers, a simple model for the arrangement of fibers in this material is needed. We are particularly interested in disordered fiber networks, as they appear in non-woven fabrics, paper, composite materials, aerosol filters and aerogels. This chapter is devoted to the description of a simple model of such networks that will allow us to include the effects of a wetting liquid by applying what we learned about capillary torques caused by liquid filaments in chapter 2

A theoretical description of such three dimensional fiber networks is relevant to many industrial processes and applications in the paper and textile industries. Fiber networks are also found in many living organisms, since their mechanical properties are flexible and easily controllable by the cell through production of crosslinking proteins. The mechanical properties of the network are determined by the elasticity of its constituents and the number of crosslinks in the system, with the latter representing the influence of the network structure on the elasticity.

The strong influence of the internal network structure on elasticity can be observed on organisms like the sea cucumber, which regulates the elasticity of its inner skin by controlling the interactions of its collagen fibrils. This inspired Capadona et al. to design a nanofibrous material whose tensile modulus can be reversibly tuned by a factor of 40 by varying the degree of linking of the fibers $\mathrm{CST}^{+} 08$.

In the context of non-woven materials such as wool, van Wyk developed a simple theory to predict the pressure during compression Wyk48, assuming that the compression consists solely in bending of the constituent fibers. Dunlop expanded this to allow for static friction and slippage events between the fibers, allowing him to predict compression hysteresis and acoustic emissions 
during compression in Dun83. Computer simulations have been performed by Beil et al. in BR02 to quantitatively calculate some parameters in van Wyk's theory.

More recently, a number of two dimensional models have been proposed. In the simplest approach, one assumes that the fibers forming the network are positioned and oriented according to a uniform distribution within a square box and along the unit circle, respectively. The crossing points are then viewed as fixed links between the fibers, leaving the angle between the fibers either unconstrained, or fixing it to a certain value. These models are commonly called Mikado models. They have been used by Heussinger and Frey to describe foams and fiber networks in [HF06b], and to characterize force chains in random networks in [HF07]. The same type of model was also used by MacKintosh and coworkers to develop an effective medium theory for fiber networks [DML07], and to study the deformation field in such a network [HLM03, LHM04].

In these models, the structural properties of the network are controlled solely by the fiber density. It is known from literature that these systems develop a finite global elasticity once the fiber density exceeds the point of rigidity percolation, where rigidly connected clusters of fibers span the whole system (see, for example, [HLM03]).

In three dimensional fiber networks, fiber entanglement may cause mechanical stiffness even without crosslinking, an effect that does not exist in purely two dimensional systems. In order to mimic this effect even in two dimensions, we distribute fixed links between fibers only with a finite probability smaller than one among the complete set of fiber crossings. We keep the motions of the fibers at the remaining crossings unconstrained.

As expected we find that the rigidity percolation threshold in terms of the fiber concentration shows a dependence on the value of the linking probability.

As described in section 2.3, each gliding contact in a wet fiber network hosts a capillary bridge which tends to align its adjacent fibers, but does not influence relative motion of the fibers in any other way. In the absence of permanent links this would cause a complete collapse of the network. This is yet another motivation to introduce permanent links along with gliding contacts. The implications of wet contacts, i.e., capillary bridges, will be explored in the following chapter 4 . 


\subsection{Crosslinkers}

In biological networks, the concept of crosslinkers emerges naturally from the fact that the individual polymer fibers, for example actin fibers in the cytoskeleton, are fixed together by crosslinking molecules. These are actin binding proteins of various kinds that are formed in the cell and typically have two ends that can both bind to actin filaments. In vitro experiments with actin and crosslinking molecules have shown that the rheological properties of these solutions depend very critically on the concentration of crosslinkers in the solution [BHE ${ }^{+}$01, TCB06, TCB07]. Cells can produce crosslinking molecules in order to fine-tune their elastic properties very efficiently. Different kinds of crosslinking molecules impose different kinds of constraints on the fibers: $\alpha$ actinin enforces a parallel orientation, while filamin leaves the crossing angle free, for example.

Another mechanism by which fibers in three dimensional networks can influence each other is a purely sterical interaction. Fibers can cross and loop around each other, making relative motion of the two fibers at the crossing point impossible because the fibers would have to penetrate each other in order to separate. Effectively, this type of interaction differs from that of crosslinking molecules only slightly: In thermal systems, entanglement can be very transient, giving rise to viscoelastic properties. On the other hand, on experimentally relevant time scales even in biological systems, the transient nature of entanglements does not play a significant role. Entangled actin networks have been studied for example in $\mathrm{KTD}^{+} 03$, $\mathrm{WGR}^{+} 04, \mathrm{GVCB03}$ experimentally, and theoretical descriptions have been proposed in $\mathrm{KTD}^{+} 03$, RFD05, HMFF08, and it has been shown that treating entanglement like a crosslink is well justified in most cases.

Entanglement naturally occurs in systems of macroscopic fibers as well. Specifically, non-woven fabrics such as paper and felt are made rigid mainly by the entanglement of their constituent fibers. Although entanglement naturally cannot occur in two-dimensional systems, it is straight forward to apply the concept of a crosslinking site that binds two crossing fibers together in order to simulate it. Studies like [MKJ95] use this approach to study entangled fiber networks in two dimensions, although the concept of treating every crossing of two fibers in two dimensions as a point of entanglement, as it is often used, may not be ideal.

Throughout this work, we will use the term crosslink or permanent link to signify a point in our two dimensional networks where we impose a constraint 
on the relative motion of two crossing fibers in order to simulate the effects of entanglement in a non-woven (i.e., disordered) fiber mat.

In the following, we will describe the most common model of a cross-linked or entangled network of fibers, and how one can infer the elastic behavior of such a network from its topological crosslinking properties.

\subsection{Random Fiber Networks: Mikado Models}

In contrast to regular lattices, the study of biological networks and non-woven fabrics is concerned with networks where the fibers are arranged without any prescribed order. The topological properties of such a network play an important role for the mechanical strength of the whole arrangement, as has been pointed out above. Therefore, many studies have utilized a simple two-dimensional model of randomly oriented fibers to study these properties. These models are sometimes called Mikado models. They all follow a simple set of rules:

The networks are initialized by depositing a number $N_{f}$ of straight fibers of fixed length $l_{f}$ in a square box of size $L$. These fibers are seen as onedimensional lines for the purpose of these models. The choice of boundary conditions varies throughout the literature, but in our work, we will use periodic boundary conditions, meaning that fibers leaving the box on one end are continued on the opposite end of the box. The positions of the fiber ends and the fiber orientations are randomly drawn from a uniform distribution. Hence, the deposition of fibers is a Poissonian process, i.e., without any spatial and angular correlations. Figure 3.1 shows an example of such a network.

In the Mikado model proposed by Frey, Heussinger and MacKintosh [HLM03, HF06b] each crossing point is decorated with a permanent link. Each of these links fixes two overlapping fibers to each other, i.e., it does not allow a relative translation of the fibers. However, it still allows them to freely rotate around the crossing point.

This uniform choice of orientations and positions leads to a certain distribution of crossing angles $\alpha \in[0, \pi]$ between fibers. Since the probability of two fibers of unit length crossing each other is much smaller when the fibers are parallel, the corresponding probability distribution is not uniform. Instead, it is given by the area of a trapezoid with edge length $1 / \sqrt{2}$ :

$$
p(\alpha)=\frac{\sin \alpha}{2} .
$$




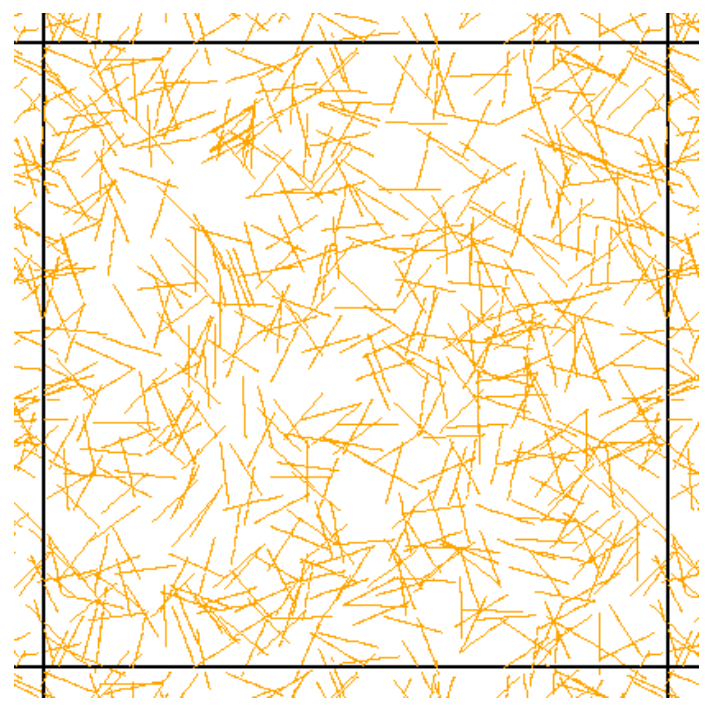

Figure 3.1: A Mikado network of 600 fibers in a box of size $L=10 l_{f}$, which therefore has a fiber density of $q=6$. The thick black lines mark the edge of the box, while outside of it periodic images are displayed.

We define the number density of fibers per unit area as

$$
q=\frac{N_{f}}{L^{2}} .
$$

This is the only control parameter governing the structure of the network. Another way to characterize the system is to calculate the average distance $\left\langle l_{c}\right\rangle$ between neighboring crossing points of fibers deposited according to the above protocol. The Poissonian nature of the deposition process implies that the distribution of crossing points on a fiber must be uniform as well. Therefore, there is an average number of $l_{f} /\left\langle l_{c}\right\rangle-1$ crosslinks per fiber. Hence, the probability density function $p\left(l_{c}\right)$, with $l_{c}$ being the actually observed distance between two crosslinks, must follow an exponential, as pointed out by Heussinger in [HSF07]:

$$
p\left(l_{c}\right)=\frac{1}{\left\langle l_{c}\right\rangle} \mathrm{e}^{-l_{c} /\left\langle l_{c}\right\rangle},
$$

with the average distance $\left\langle l_{c}\right\rangle$. The average distance itself is related to the fiber density $q$ as follows:

$$
\left\langle l_{c}\right\rangle=\frac{\pi l_{f}}{q}=\frac{\pi L^{2}}{N_{f} l_{f}} .
$$


In the limit of infinite system sizes $L$, the average crosslinker distance $\left\langle l_{c}\right\rangle$ and the fiber length $l_{f}$ are the two only length scales in a Mikado model. While $\left\langle l_{c}\right\rangle$ governs the distances between the crossing points constraining the motion of fibers, stiff fibers still maintain their identity across a freely rotating link over the whole length of $l_{f}$.

In contrast to the standard Mikado model, we will introduce a finite probability $p_{l}$ with which a crossing point is converted into a permanent link. Apart from the fact that the sites where capillary interaction takes place are always single contacts where the touching fibers can glide across each other, this is fully justified by the fact that in real systems, not every fiber contact will be a point of entanglement. For random assemblies of thin rods in three dimensions, Philipse [PV97] calculated the probability that a rod is caged as a function of the packing fraction, coming to the conclusion that an average of 5 contacts is necessary to suppress the translations of a rod. Rodney et al. RFD05] found in computer experiments that the number of contacts needed for entanglement varies between 4 and 8, depending on the fiber aspect ratio. Mikado models with $p_{l}=1$ therefore are not accurate descriptions of entangled three-dimensional fiber mats.

Mikado models have been shown to exhibit different elastic regimes depending on the average distance of crosslinks, $\left\langle l_{c}\right\rangle$, which in turn is related to the fiber density $q$ by equation 3.4. This is described in detail in Heussinger et al. [HSF07].

MacKintosh and coworkers summarize the influence of the network density on the elastic properties as follows [HLM03, LHM04]: At very low concentrations, the fibers are not globally connected to each other, and the system behaves as a fluid with zero shear modulus. Upon increasing the concentration of fibers, a global cluster of connected fibers forms, meaning that there is a percolation transition of fiber connectivity. When the system is thermal, i.e., when it has a nonzero temperature and therefore entropic contributions to the free energy have to be considered, global connectedness means that an externally imposed shear that moves all the nodes of the network will lessen the total number of configurations that a fiber between two such nodes can take. Thus, work has to be done in order to shear the system, and the shear modulus is non-zero.

On the other hand, systems of macroscopic fibers stretch and bend at energy scales far above that of the ambient temperature. They are therefore not subject to thermal fluctuations. In such athermal systems, there is no 
energy cost associated with a relative motion of the nodes as long as the fibers stretched between those nodes do not need to stretch or bend in order to accommodate it. Instead, the fibers can rotate around the crossing points without cost of energy. Only when a sufficient number of further constraints in the form of further crosslinks is added do these floppy modes of motion become suppressed. Thus, in athermal systems, there is a rigidity percolation transition that is different from the percolation of connectivity.

Once a critical value $q^{*}=(6.67 \pm 0.02)$ of the fiber density is reached, a globally rigid cluster of fibers forms that can transfer shear forces across the system [LKT01]. For concentrations slightly above the critical point the shear modulus behaves as

$$
G \propto\left(q-q^{*}\right)^{f}
$$

with $f \approx 3.0$ HLM03.

In this regime (with $q$ slightly above $q^{*}$ ), the elasticity of the network is governed by topological properties of the fractal percolation cluster. It is this regime with its strong dependence on $q$ that enables cells to control their elastic moduli via crosslinking molecules. In section 3.5 we will further study this regime for the specific case of $p_{l}<1$, which was not previously done.

Increasing $q$ further, the elasticity becomes independent of the system size, since the divergence of the size of the percolating cluster is now complete. In this regime, the deformations are dominated by fiber bending. Heussinger et al.[HF06a, [HSF07] identified this regime with the existence of low-energy bending modes by which the system can lower its elastic energy when an external deformation is imposed. These modes dominate the elastic response when the typical bending length

$$
l_{b} \approx \sqrt{\frac{\kappa}{\mu}}
$$

is smaller than the distance between crosslinks $\left\langle l_{c}\right\rangle$. In the above equation, $\kappa$ is the bending rigidity of an individual fiber and $\mu$ is its stretching modulus.

Once the density $q$ is high enough (and therefore $\left\langle l_{c}\right\rangle$ low enough) that a fiber cannot bend significantly between crosslinks, the network behaves more or less like a continuum solid: The microscopic deformations are homogeneous and equivalent to the macroscopic deformation (this is called affine deformation), and the segments must stretch to accommodate the external strain. In this regime, the shear modulus is mainly dependent on the individual segments' stretching rigidity $\mu$. 
For this region, Head et al. [HLM03] have pointed out that since all fibers deform affinely, the shear modulus can be calculated from averaging the elastic energy of one deformed fiber over all fiber orientations. They have also identified the crossover from non-affine to affine deformations and described it via the parameter

$$
\lambda=\left\langle l_{c}\right\rangle\left(\frac{\left\langle l_{c}\right\rangle}{l_{b}}\right)^{z},
$$

where the exponent $z \approx 1 / 3$. According to [HLM03, the deformations become affine at the point where $l_{f} / \lambda$ is of the order 10 .

In the rest of this chapter we will concentrate on the region of fiber densities where the percolation transition governs the elasticity, since we expect that it is this low-density regime where internal stresses such as those caused by capillary interaction have the largest influence on the structure (and therefore the elasticity) of the network.

\subsection{Topological Rigidity}

This section will briefly explain the physics of the most important density regime of elastic fiber networks: That of the rigidity percolation transition, where elastic properties depend mostly on the network topology. This is the regime of lowest fiber densitites that still exhibits global rigidity. It is therefore also the most intersting density regime for wet fiber networks, since the low density makes rearrangements of the structure through the action of liquid bridges possible, while the network still offers resistance to the capillary action.

In this regime, the physics are governed by the percolation transition. Therefore, we will first introduce the fundamental concepts behind percolation using the example of bond percolation.

\section{Bond Percolation}

The theory of bond percolation typically deals with regular lattices. There, one asks the question whether such a lattice has an open path connecting its ends when each edge or "bond" of the lattice is said to be "open" with a fixed probability $p$. The existence of such an open cluster could for example mean that a porous material lets liquid flow through it, when the open bonds represent connected pores in the material. Consider the example in figure 3.2 . It shows a square lattice where only bonds marked as open are shown. The 


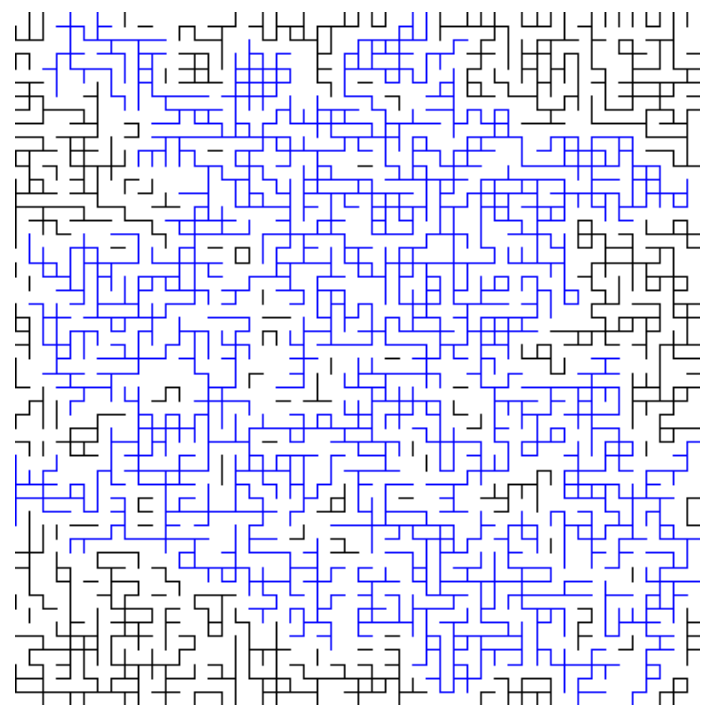

Figure 3.2: A bond-diluted square lattice at $p=0.51$, with the percolating cluster marked in blue. Image source: Wikipedia

probability of marking a bond open was chosen as $p=0.51$ in this case. Shown in blue is the largest cluster of connected open bonds. It can be seen that this cluster spans the system and permits liquid to flow from top to bottom. Such a cluster is said to be percolating.

The study of percolating clusters is first introduced in [BH57] and thoroughly explained in [SA95].

Consider a network or lattice with a prescribed topology (either regular or random, and with a certain coordination number) and with a certain size $L$ signifying the number of bond lengths of one edge of the box. When each bond in this network is independently marked open with probability $p$, the probability of existence of a percolating cluster is a function of $p$ and $L$ only.

One is usually interested in the limit case of $L \rightarrow \infty$. In this case, the probability of such a cluster existing is either zero or one, and there is a critical value $p_{c}$ above which an infinite cluster always exists.

In the example of the square lattice, one finds that $p_{c}=0.5$ [SA95]. In a Mikado model, the fiber density $q$ plays the role of $p$, and the point where a connected path of fibers throughout the system exists has been shown to lie at $q_{c}=5.71$ [PS74].

To quantify the largest cluster of open bonds in the system, one can measure the number $M$ of bonds in a subsystem of size $L$ that belong to a certain 
cluster. Then,

$$
P=M(L) / L^{2}
$$

gives the density of bonds in the system that belong to this cluster.

Measuring $M(L)$ for different realizations of networks and different values of $p$ close to $p_{c}$ in computer experiments shows that it scales as a power law of the system size $L$ :

$$
M(L)=L^{D}
$$

with exponent $D$. This means that the percolating cluster at $p=p_{c}$ is fractal with fractal dimension $D$. Above $p_{c}$, the exponent $D$ becomes equal to the dimension of the system, meaning that the cluster has a homogeneous density and is not fractal.

Another important quantity is the correlation length of the cluster. It is defined via the two-point correlation function $g(r)$ that measures the probability that two bonds that are a distance $r$ apart are part of the same cluster. Computer experiments show that it decays exponentially as

$$
g(r)=\exp \left(\frac{-r}{\xi}\right)
$$

where $\xi$ is called the correlation length and is a typical length scale of the system at a given $p$. When $p$ approaches $p_{c}$, the correlation length $\xi$ is observed to diverge [SA95]:

$$
\xi \propto\left(p-p_{c}\right)^{-\nu}
$$

Here $\nu$ is the exponent with which this lengths scale diverges at $p=p_{c}$, which must be measured in computer experiments.

A suitable observable to judge the percolation of a system is the density $P_{\infty}$, defined as the density $P$ of the largest cluster. When $p<p_{c}$, the largest cluster still has a finite size, and thus in an infinite system $P_{\infty}$ must be zero. Then as $p \rightarrow 1$, the density of the largest cluster must also approach one. Measurements show that $P_{\infty}$ follows a power law as well for $L \gg \xi[$ SA95]:

$$
P_{\infty} \propto\left(p-p_{c}\right)^{\beta}
$$

this time with a different exponent $\beta$. 
To measure the percolation point in computer experiments with finite systems, the behavior of these quantities at finite system sizes $L$ must be addressed. Equation (3.9) shows that for $L<\xi$, the mass of the largest cluster inside such a subsystem scales as $L^{D}$. Above the correlation length, however, this cannot hold anymore. When one further subdivides the box into boxes of size $\xi$, there are $(L / \xi)^{2}$ boxes of this kind, while within each box the mass still scales as $\xi^{D}$. So we find the following scaling behavior:

$$
M(L, \xi)= \begin{cases}L^{D} & L \ll \xi \\ \xi^{D}\left(\frac{L}{\xi}\right)^{2} & L \gg \xi\end{cases}
$$

Since the density $P$ of the cluster is $P=M L^{2}$, and since equation (3.13) states that $M \propto \xi^{D-2} L^{2}$, it follows that for $L>\xi: P \propto \xi^{D-2}$.

In other words, for $L \gg \xi$ the mass $M$ depends on $L$ only, while for $L \ll \xi$ any corrections can only be a function of $L / \xi$ since $\xi$ is the only measure of length in the system.

Using the scaling equations (3.11) and (3.12), the behavior of $P_{\infty}$ becomes

$$
P_{\infty}=\left\{\begin{array}{ll}
L^{-\frac{\beta}{\nu}} & L \ll \xi \\
\xi^{-\frac{\beta}{\nu}} & L \gg \xi
\end{array} .\right.
$$

It is common to express (3.14) and (3.12) in terms of a scaling function:

$$
P_{\infty}=\left(p-p_{c}\right)^{-\beta} f\left(\left(p-p_{c}\right) L^{\frac{1}{\nu}}\right),
$$

here $f(x)$ is the scaling function that satisfies $f(x) \rightarrow 1$ for $x \gg 1$ and $f(x) \rightarrow(L / \xi)^{\beta / \nu}$ for $x \ll 1$. This form of scaling function must be assumed for all quantities that scale as $\left(p-p_{c}\right)^{\beta}$.

This shows that at finite $L$, the observed point of percolation (i.e., the inflection point of the curve $\left.P_{\infty}(p)\right)$, will depend on $L$. When it is plotted against $L^{\frac{1}{\nu}}$, the percolation point $p_{c}$ can be extrapolated. We will use this method of finite size scaling in section 3.5 when we investigate rigidity percolation in dilute Mikado models with different linking probabilities $p_{l}<1$.

\subsubsection{Rigidity Percolation}

When one measures physical quantities on percolating networks, such as for example the total resistance of a random square lattice where each bond is a conductor of unit resistance, one will find different exponents than those 
presented for, e.g., the mass of the percolating cluster. The reason for this is that not all bonds in the network have the same weight in the calculation of the resistance: Some resistors can be redundant.

A similar reasoning holds when measuring the shear modulus of a system of connected fibers. Here, a different effect is added: Even if a network has a percolating cluster of connected fibers, the shear modulus may be zero, since the fibers can rotate around their crossings without deforming. Only when enough crossings exist that these deformation modes cannot accommodate the given shear deformation does the shear modulus become finite. Heussinger et al. [HSF07] give a nice illustration of these floppy modes.

So, coming back to the question of rigidity percolation, one must define a cluster in the system not as an arrangement of connected fibers, but only as that type of arrangement that is mechanically rigid. In the following section, we will present a simple estimation method for the rigidity percolation point, while section 3.4 will give a more thorough computational approach.

\subsubsection{Maxwell Counting}

Maxwell proposed a simple method to determine percolation in networks of beams Max64 by counting degrees of freedom.

Considering first the connectivity percolation, the task is to find the fiber density $q_{c}$ at which all fibers in an infinite system are connected.

In this picture, one fiber represents one degree of freedom (a fiber is either connected or not), and a crossing represents one constraint (it connects two fibers). In Maxwell's approximation, percolation happens when the number of degrees of freedom equals the number of constraints.

Since there are $N_{f}=q L^{2}$ fibers in the system, and, according to equation (3.4), the number of crosslinks $N_{c}$ is

$$
N_{c}=N_{f} \frac{l_{f}}{\left\langle l_{c}\right\rangle} \stackrel{3.4}{=} \frac{N_{f} q}{\pi},
$$

equating $N_{f}$ and $N_{c}$ and solving for $q$ leads to

$$
q_{c}=\pi
$$

In diluted Mikado networks, the expression for $N_{c}$ would have to be multiplied by $p_{l}$, so that here

$$
q_{c}=\frac{\pi}{p_{l}} .
$$


However, computer simulations show that in fact $q_{c}=5.71$ for $p_{l}=1$ (see [PS74]).

The reason for this is that Maxwell counting underestimates $q_{c}$ because it supposes that all constraints are independent, i.e., that every crosslink connects two fibers that have not already been connected by some path. It could in principle also overestimate $q_{c}$ because not all fibers need to be connected to have a percolating cluster. Hence, Maxwell counting can neither give an upper nor a lower bound for $q_{c}$, only a rough estimate.

The case of rigidity percolation can also be addressed by Maxwell counting. Here, the degrees of freedom under consideration are the actual modes of motion that a rigid fiber can undertake. Since a fiber in two dimensions has one rotational and two translational degrees of freedom, the total number of degrees of freedom is $3 N_{f}$.

Fixed links of the type we introduced in Mikado models bind two degrees of freedom, the two relative translational motions of the fibers, while leaving one degree, the rotations around the crossing point. Hence, the number of constraints is $2 N_{c}$, and the point of rigidity percolation becomes

$$
q^{*}=\frac{3 \pi}{2 p_{l}}
$$

However, as we have seen above for the connectivity percolation, this value may over- or underestimate the true value of $q^{*}$ by some amount. Therefore, it is necessary to determine the actual number of degrees of freedom that are bound by crosslinks in a Mikado network computationally.

Several studies address the rigidity percolation of regular lattices by means of explicitly calculating the elastic energy of the bonds and thus measuring the shear modulus, determining the point of percolation $p^{*}$ and the exponent with which the modulus vanishes at $p=p^{*}$. For example, Feng and Sen[FS84 find for the two dimensional triangular lattice of Hookean springs: $p_{c}=0.58$. However, the approach of purely Hookean springs does not take bending deformations into account.

For random Mikado-type networks with bending forces, Lava-Kokko and Timonen had more success with a sophisticated method to count the degrees of freedom instead of measuring elastic energies, which enabled them to address much larger systems. They find a value of $q^{*}=6.67$ for $p_{l}=1$ [LKMT01].

In the following, we will use the same approach for diluted Mikado networks with $p_{l}<1$. To that end, we will first describe the algorithm proposed by 
[JT96] and used in [LKMT01] to characterize the rigidity percolation, and then present our own results in section 3.5 .

\subsection{The Pebble Game}

Identifying rigid clusters is not trivial since rigidity is non-local: A fiber connecting two nodes can constrain the motions of other nodes that are far away. For example, a large cluster of simply connected fibers can be made rigid by adding fibers at faraway points in the cluster.

We will now present a method to identify rigidly connected clusters of fibers in a crosslinked network. This method is known as the Pebble Game algorithm and has been introduced by Jacobs and Thorpe [JT95, JT96] to study rigidity percolation on the triangular lattice. It has also been used by Latva-Kokko and Timonen [LKMT01] to study Mikado networks of fibers with stretchingand bending rigidity in the case of $p_{l}=1$.

The restriction to analyzing only the network topology, not the elastic energies, means that certain accidental symmetries are not taken into account. Take for example a set of nodes connected by independent fibers in a way that the nodes are all collinear. This contrived situation will not actually occur in a Mikado network, since only two fibers ever cross at the same crossing point, but it will serve to illustrate this type of geometrical singularity. When one tries to stretch such an assembly, the only motion the nodes can take to accommodate that is a stretching of the fibers, hence the system is rigid. However, if one wants to compress the line of fibers, the fibers can rotate around the crossing points to accommodate the approaching of the outermost nodes. This is an example of a buckling instability. This asymmetry does not occur if the nodes are shifted randomly from their collinear positions by a small amount. Then, both stretching and compression are floppy.

Another example of such a geometric singularity is illustrated in figure 3.3 . Again, a random displacement of the nodes by a small amount destroys the symmetry and makes the structure rigid. Since the set of networks containing such accidental symmetries is of measure zero, one will never encounter them in a random Mikado network.

Since the Pebble Game only deals with the topology of the network, it makes statements about the rigidity of the generic version of the network studied, where generic is taken to mean a topologically equivalent version of the network without geometric singularities, e.g., one where all nodes have 


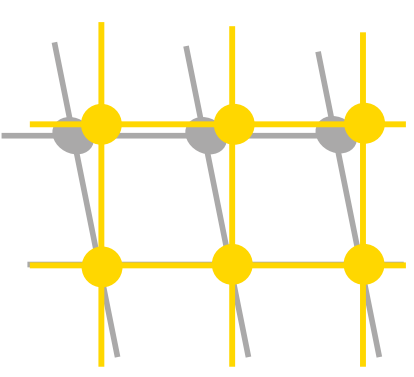

(a) floppy

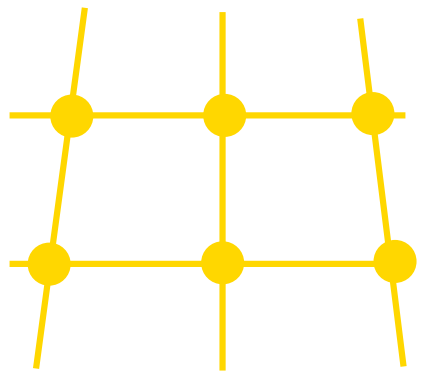

(b) rigid

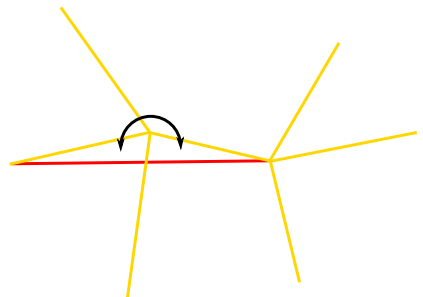

(c) second-nearest neighbor bonds

Figure 3.3: Two geometrically different realizations of a topologically rigid cluster. The symmetric realization (a) has a floppy mode (indicated by the gray cluster) that arises only from its symmetry. The generic version (b) cannot undergo this deformation without stretching the central bond. Figure (c) shows the implementation of bending stiffness: The yellow bonds represent flexible fibers, with nodes at the crossing points. The red bond is a second-nearest neighbor bond that imparts bending stiffness to the horizontal fiber: Bending of that fiber at the node marked by the arrows will always deform the red bond. Shown here is the generic version of the Mikado network with a second-nearest neighbor bond; in the straight version, bending would be a soft mode.

been displaced by a small and random vector.

This also means that the bending rigidity of the fibers can be implemented in a simple way: When one introduces second-nearest neighbor bonds connecting nodes of the same fiber across every crosslink, bending of the fibers is always associated with a deformation of these second-nearest neighbor bonds. This is illustrated in figure $3.3(\mathrm{c})$,

Because of this, we will use the term bond to mean each connection between fixed links in the network, be it a fiber or a virtual bond introduced across a link to give the fiber bending rigidity.

The Pebble Game assigns the degrees of freedom of the network to the crosslinks or nodes. Each node has two degrees of freedom in two dimensions. Each bond connecting two nodes binds one degree of freedom: The distance between the two nodes. The algorithm is based on the theorem of Laman from graph theory [Lam70]: 


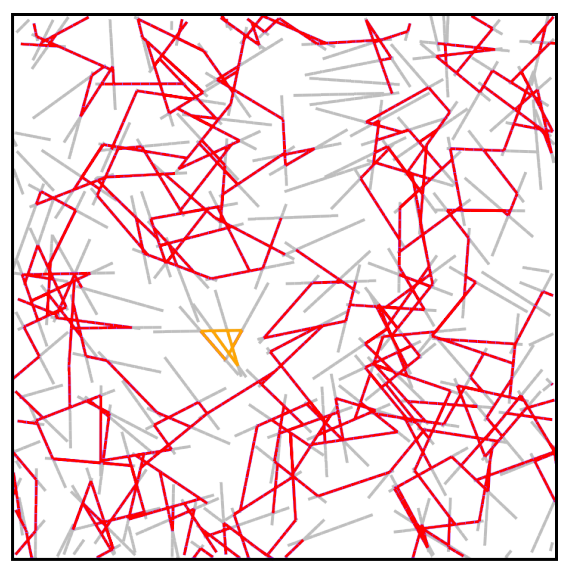

(a)

Figure 3.4: A Mikado network of 280 fibers at $q=7$. Rigidly connected clusters are marked in color, while fibers not contributing to the network rigidity, including dangling bonds at the ends of fibers, are gray. The percolating cluster is shown in red. Also visible is a smaller rigid cluster in yellow.

Theorem 3.1 A generic network in two dimensions with $N$ nodes and $B$ bonds does not have a redundant bond if no subgraph of the network containing $n$ nodes and $b$ bonds violates $b \leq 2 n-3$.

A redundant bond is a bond that does not bind degrees of freedom, since the distance of the nodes it connects is already fixed by other constraints. It can be seen by simple Maxwell counting that a redundant bond must exist when the Laman condition is violated.

The algorithm works by building up a network bond by bond. Whenever a bond is added, all subgraphs are checked for the Laman condition to determine whether the bond is redundant or not. This is done by representing the degrees of freedom with pebbles. These are imaginary objects associated with the degrees of freedom of motion of the nodes. A pebble can be either free, and attached to a node, signifying that this node has one degree of freedom. Or the pebble can be bound to a bond, signifying that the bond restricts the motion of the nodes it connects by one degree of freedom (since it fixes their distance). Initially, each node has two free pebbles attached to it representing its two degrees of freedom.

When inserting a bond, one must first determine whether it is independent. 
If the two nodes it connects have two free pebbles each, then the distance between these nodes is not yet fixed. This means that the added bond is independent. According to the rules of the Pebble Game, all independent bonds must be covered by one pebble. This pebble is taken from any of the connected nodes and is now no longer considered free.

When another bond is added, it must again be checked for independence. If the two adjacent nodes do not initially have two free pebbles each, it could still be possible to free one of their pebbles that is bound to an independent bond by covering that bond with a free pebble from another node attached to it. This is the way the search over the subgraph is performed in the Pebble Game algorithm.

When it is not possible to free four pebbles at the ends of a newly added bond, then that bond is redundant. The set of bonds that was unsuccessfully searched for a free pebble constitutes an overconstrained region in the network, a subgraph that violates the condition in theorem 3.1. Any bond added to such a subgraph will be redundant, so that this subgraph need not be searched again.

After the network is complete, it can be segmented into rigid clusters. To do this, bonds are sequentially chosen as reference bonds. Then, a virtual test bond is added between one of the nodes of the reference bond and a second node. If the rules of the Pebble Game state that the test bond is redundant, then that node is rigid with respect to the reference bond. Since a bond can only belong to one cluster, all nodes found to be rigid with respect to the reference bond belong to the same rigid cluster.

Figure 3.4(a) shows a small Mikado network with two identified rigid clusters. In the following, we will show our results of applying the Pebble Game algorithm to diluted Mikado networks to find the density $P_{\infty}$ of the percolating rigid cluster, and thus to identify the rigidity percolation point.

\subsection{Results: Rigidity Percolation of Diluted Mikado Networks in 2D}

To analyze the topological rigidity of a Mikado model network with finite $p_{l}$, we generate a number of such networks, adding fixed links at crossing points randomly with probability $p_{l}$. Then, we add second-nearest neighbor links across the crosslinks, so that the generic network will be equivalent to a Mikado model network with fibers of finite bending rigidity. 
Then we employ the pebble game algorithm, segmenting the network into rigid clusters and identifying the largest cluster. The percolation probability $P_{\infty}$ of this is then equal to the number of bonds in the cluster divided by the total number of bonds.

This measurement was performed for system sizes $L=(50,60, \ldots, 100) l_{f}$, for various densities $q$ and for the different crosslinking probabilities $p_{l}$. For each combination of parameters, 50 random networks were generated and analyzed in order to derive the average probability $P_{\infty}\left(q, L, p_{l}\right)$. Figure 3.5 shows plots of $P_{\infty}$ versus $q$ for varying system sizes and linking probabilities.

As can be seen, the probability of having a percolating cluster goes from zero to one on increasing the fiber density. The transition is sharper at larger system sizes $L$. This was expected from the scaling relation (3.15). We identify the inflection point, that is, the point where the percolation transition appears to happen at a certain system size, as the point where $P_{\infty}=1 / 2$.

For $p_{l}<1$, the probability of a bond being in the largest cluster becomes smaller instead of larger with smaller system sizes. This is an indication that the fractal nature of the percolating cluster changes: When dividing a large system with a percolating cluster into subsystems, the probability of a subsystem containing a percolating cluster is now smaller. One possible reason for this is that at $p_{l}<1$, several system-spanning rigid clusters can overlap, since overlap does not imply linking. On increasing the system size, the probability that these clusters form a sufficient number of links between them goes up, so that they can combine into one percolating cluster that only appears as a single cluster at large system sizes. Therefore, the probability $P_{\infty}$ in less dense systems can grow with the system size.

From the curves in figure 3.5, the transition points $q_{1 / 2}$, defined as the points where $P_{\infty}=\frac{1}{2}$, have been extracted. They are shown in table 3.2. Expanding equation (3.15), interpreted in terms of the fiber density, around the percolation point $q=q^{*}$, one finds

$$
q_{1 / 2}(L)=\text { const } \cdot L^{-\frac{1}{\nu}}+q^{*} .
$$

This equation holds for any fixed value of $q$ between zero and one, but we chose $1 / 2$ because it gives the sharpest definition of a percolation point. So, fitting a power law to $q_{1 / 2}(L)$ and then plotting it against $L^{\frac{1}{\nu}}$ gives us the percolation point $q^{*}$ at infinite system size. This has been done in figure 3.6.

From these fits, the infinite size limit of the percolation point, $q^{*}$, can be extracted. These points are shown together with the fitted exponents $\nu$ in table 3.1 . 


\begin{tabular}{ccc}
\hline $\boldsymbol{p}_{\boldsymbol{l}}$ & $\boldsymbol{q}^{\boldsymbol{*}}$ & $\boldsymbol{\nu}$ \\
\hline 0.4 & $7.84 \pm 0.04$ & $0.93 \pm 0.06$ \\
0.5 & $7.75 \pm 0.02$ & $0.92 \pm 0.05$ \\
0.7 & $7.74 \pm 0.01$ & $0.86 \pm 0.04$ \\
0.9 & $7.69 \pm 0.01$ & $0.85 \pm 0.02$ \\
1.0 & $6.676 \pm 0.001$ & $0.851 \pm 0.002$ \\
\hline
\end{tabular}

Table 3.1: Percolation points $q^{*}$ for different linking probabilities $p_{l}$.

As can be seen there, lower linking probabilities require a higher fiber density to achieve rigidity percolation, as has been expected, for example, from the constraint counting argument $(3.19)$. Also, due to the change in the fractal structure of the rigid clusters at $p_{l}<1$, the correlation length exponent $\nu$ increases by a small amount.

The values for $p_{l}=1$ correspond well with the value of 6.6 that Latva-Kokko and Timonen have calculated in LKT01 using much the same method.

From looking at figure 3.6, it seems that, while the change in scaling exponent and percolation point is systematic and monotonic with varying $p_{l}$, the case of $p_{l}=1$, i.e., the classical dry mikado network case, is quantitatively different. The reason for this is probably in the qualitative change in the fractal structure introduced by the random linking rule. Exploring this further is beyond the scope of this work.

However, it is to be noted that the wet fiber networks examined numerically in this work are of necessity far away from the limit case of infinite $L$ examined here, since large system sizes and fiber densities would impose prohibitive computational costs. Therefore, it is recommended to refer to table 3.2 and figure 3.5 for the specific system sizes and linking probabilities given as simulation parameters when judging the topological rigidity of simulated networks. In particular, the percolation point for $p_{l}=0.5$ and $L=12$ has been added to table 3.2 , since this is the most frequently used parameter set. 


\begin{tabular}{rrc|rrc|rcc}
\hline $\boldsymbol{p}_{\boldsymbol{l}}$ & $\boldsymbol{L}$ & $\boldsymbol{q}_{1 / \mathbf{2}}$ & $\boldsymbol{p}_{\boldsymbol{l}}$ & $\boldsymbol{L}$ & $\boldsymbol{q}_{1 / \mathbf{2}}$ & $\boldsymbol{p}_{\boldsymbol{l}}$ & $\boldsymbol{L}$ & $\boldsymbol{q}_{1 / \mathbf{2}}$ \\
\hline $\mathbf{0 . 4}$ & 50 & $9.4 \pm 0.4$ & $\mathbf{0 . 7}$ & 50 & $7.9 \pm 0.2$ & 0.9 & 50 & $7.7 \pm 0.2$ \\
& 60 & $9.1 \pm 0.6$ & & 60 & $7.9 \pm 0.3$ & & 60 & $7.7 \pm 0.2$ \\
& 70 & $8.8 \pm 0.4$ & & 70 & $7.8 \pm 0.3$ & & 70 & $7.7 \pm 0.1$ \\
& 80 & $8.6 \pm 0.3$ & & 80 & $7.8 \pm 0.2$ & & 80 & $7.7 \pm 0.1$ \\
& 90 & $8.4 \pm 0.8$ & & 90 & $7.8 \pm 0.3$ & & 90 & $7.69 \pm 0.05$ \\
& 100 & $8.3 \pm 0.4$ & & 100 & $7.7 \pm 0.2$ & & 100 & $7.69 \pm 0.05$ \\
$\mathbf{0 . 5}$ & 50 & $8.7 \pm 0.3$ & $\mathbf{1 . 0}$ & 50 & $6.6 \pm 0.2$ & & & \\
& 60 & $8.5 \pm 0.4$ & & 60 & $6.7 \pm 0.2$ & & & \\
& 70 & $8.3 \pm 0.5$ & & 70 & $6.66 \pm 0.09$ & & & \\
& 80 & $8.2 \pm 0.4$ & & 80 & $6.66 \pm 0.09$ & & & \\
& 90 & $8.1 \pm 0.2$ & & 90 & $6.67 \pm 0.08$ & & & \\
& 100 & $8.0 \pm 0.3$ & & 100 & $6.68 \pm 0.07$ & & & \\
$\mathbf{0 . 5}$ & 12 & $9.4 \pm 0.5$ & $\mathbf{0 . 7}$ & 12 & $8.6 \pm 0.4$ & & & \\
\hline
\end{tabular}

Table 3.2: Inflection points of the percolation transitions at finite sizes, given for different system sizes $L$ and linking probabilities $p_{l}$, as derived from the curves in figure 3.5. The bottom row gives the inflection points for the system sizes used in simulations in chapter 4. 


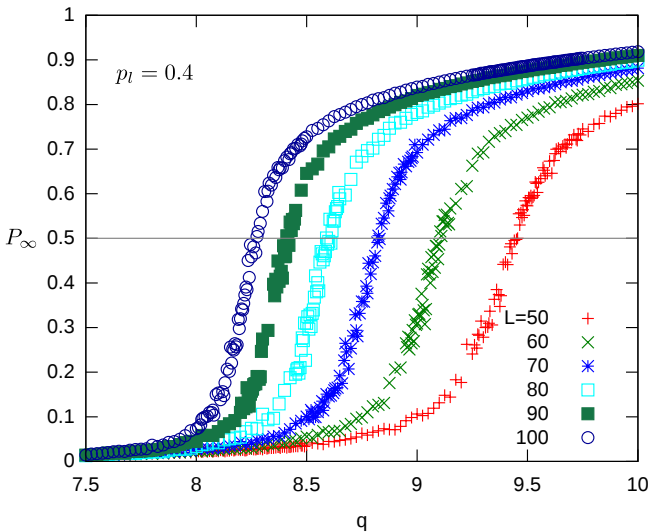

(a)

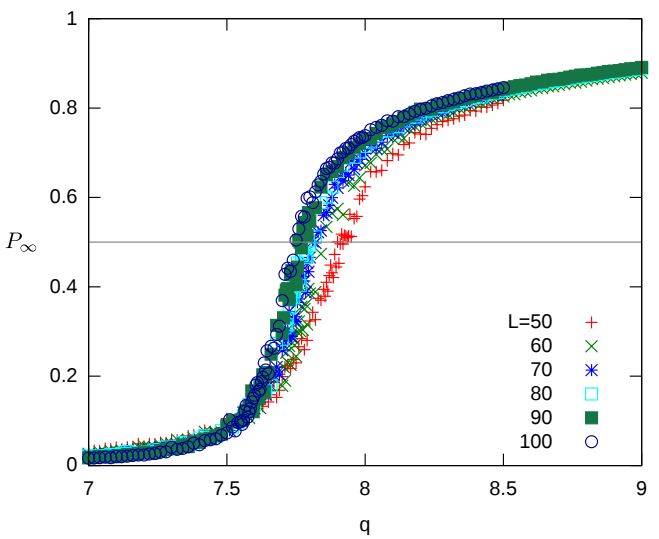

(c)

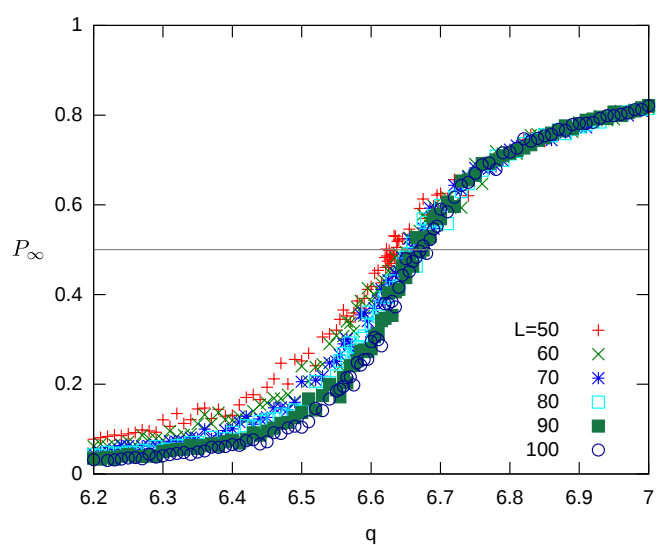

(e)

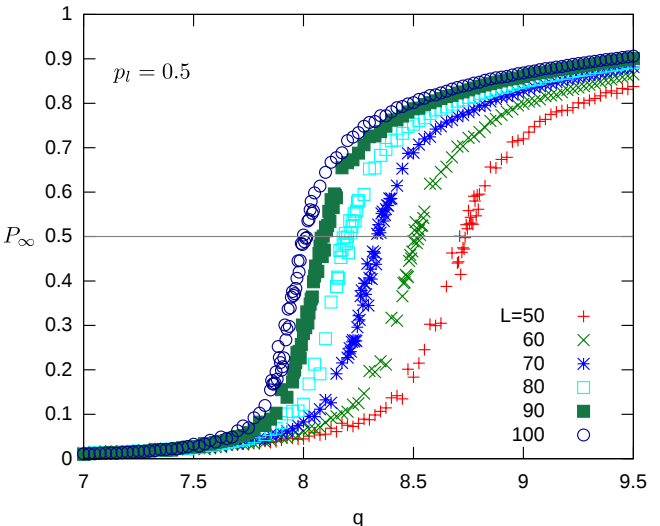

(b)

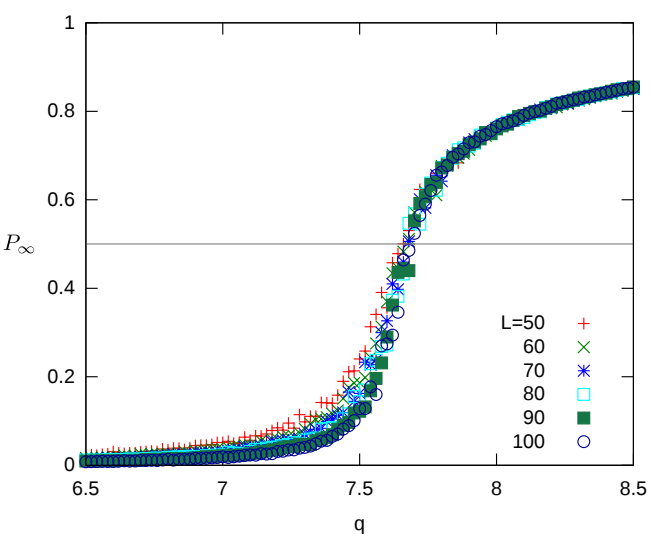

(d)

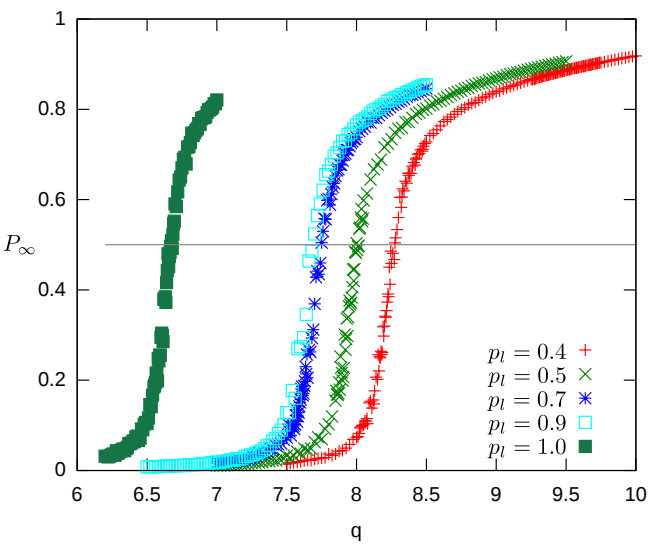

(f)

Figure 3.5: The relative mass $P_{\infty}$ of the largest rigid cluster is plotted for several system sizes, and at various crosslinking probabilities $p_{l}$. $(\mathrm{a}): p_{l}=0.4(\mathrm{~b}): p_{l}=0.5(\mathrm{c}): p_{l}=0.7(\mathrm{~d}) ; p_{l}=0.9(\mathrm{e}): p_{l}=1.0$. Figure (f) shows all the curves for the largest system size on the same $q$-axis. 


\subsection{Conclusions}

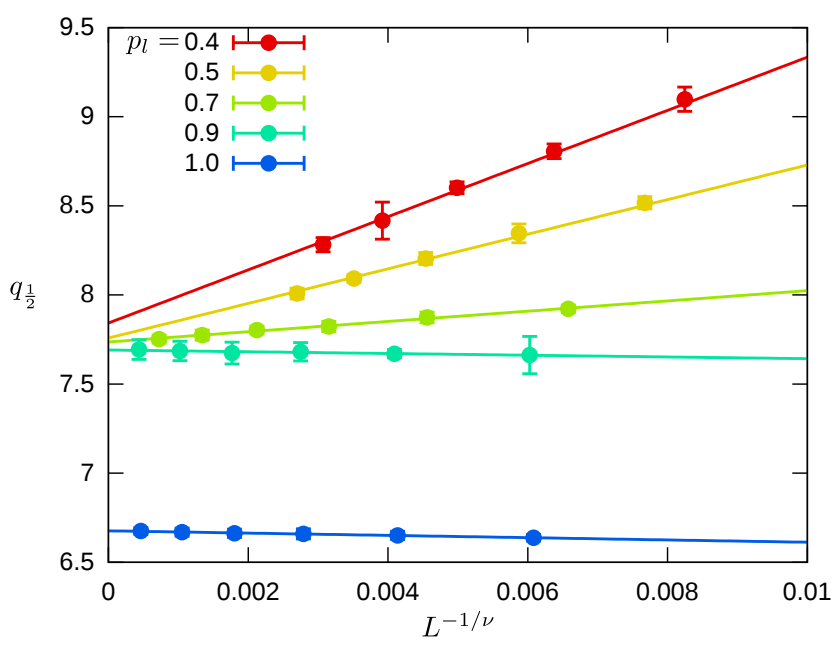

Figure 3.6: Finite size scaling of the percolation point for different crosslinking probabilities $p_{l}$. The inflection point density $q_{1 / 2}$ is plotted against the system size $L$, rescaled with an exponent $\nu$. The values for the exponents and offsets can be seen in table 3.1 .

In this chapter, we introduced the concept of Mikado model networks, which represent a common model system to analyze a range of different physical fiber networks analytically and computationally. We expanded this concept by introducing a finite linking probability $p_{l}$, which should give a better description of real entangled fiber networks and non-woven fabrics, as well as allowing us to introduce new kinds of interaction in rigid network structures at the remaining crossing points.

Since we pointed out that the topology of the crosslinked network is known to have a very large influence on network rigidity, we then focused on analyzing the influence of this finite $p_{l}$ on topologically induced rigidity. It was found, as can be expected, that for $p_{l}<1$ much higher fiber densities are required in order to generate a globally rigid network. This effect could be quantified by using the pebble game algorithm by Jacobs and Thorpe.

With the ingredients introduced so far, i.e., the quantitative analysis of capillary torques between fibers in chapter 2, and the diluted Mikado model in this chapter, it will be possible to develop a model for wet fiber networks. The question of what value of $p_{l}$ to choose for such a model remains to be 
answered. Since there is no apparent way to derive a value of $p_{l}$ that would represent a realistic three dimensional fiber network in a Mikado model, and since the number of system parameters is already very large, we will choose $p_{l}=0.5$ throughout the rest of this work. 


\section{Chapter 4}

\section{Wet Fiber Networks}

In this chapter, we introduce a computer model for crosslinked elastic fibers that is capable of describing the elastic deformation of the fibers themselves and the dynamics of such a system. This is in contrast to the previous chapter, where we only considered the modes of deformation of a network that leave the individual fibers undeformed.

Now to extend this towards a model where elastic deformations of the fibers can be tracked in computer simulations, we will introduce a discretized description of deformed cylindrical fibers and their bending and stretching energies, which we will then apply to a bead-string type model to calculate the dynamics of such a fiber. This introduces an additional distinction between nodes that merely connect two segments of a discretized fiber, and nodes that represent crosslinks between fibers as described in chapter 3. We will call the latter supernodes. Ideally, there will be many simple nodes between supernodes, so that deformations of the fiber segments between crosslinks can be taken into account.

Once this is done, we introduce a way of modeling capillary adhesion in this kind of system by introducing a mobile crosslink-like object that can move along the fibers and that exerts forces on them that are equivalent to the capillary forces experienced by wet cylinders.

With this computer model, we will study structure formation and mechanical properties of wet fiber networks. It enables us to examine the growth of pores driven by capillary forces, which is very relevant to technical application such as aerosol filters and the resistance of aerogel materials to humidity. 


\subsection{Discrete Element Model for Elastic Fibers}

In this section, we will present a discretized model to describe the elastic deformations of cylindrical fibers in a network.

Under the assumption that the non-dimensional thickness, curvature and twist of an elastic fiber, as well as the strains, are small, the first order elastic theory by Kirchhoff (see[Kir59, LL86, Dil92, $\mathrm{CDL}^{+}$93]) can be used to describe an elastic rod.

Kirchhoff's theory still allows for large rotations, meaning that over the length of a rod, large deflections can accumulate. The requirement of small twist need not concern us here, since the model presented here is two-dimensional, and fibers restricted to a plane do not have twist. Kirchhoff describes the rod based only on differential-geometrical properties of the curve described by the fiber's center line. Thus, the cross sections are assumed to remain plane and orthogonal to the centerline on deformation. These assumptions are justified when the rods are sufficiently slender.

In its undeformed state, a rod in this type of theory is an elongated object, small in two dimensions as compared to the third, extended dimension. The cross sections of this object are uniform along its length. The center line of the rod is described by a space curve $\mathbf{r}(s)$ with $s$ parametrizing the material points of the rod. This then gives the unit tangent vector of the rod as $\mathbf{t}(s)=\mathrm{d} \mathbf{r} / \mathrm{d} s$. Together with two orthonormal vectors $\mathbf{a}_{1,2}$, this forms a basis for the material points of the rod.

When the rod is deformed from its initial state, its relative extension $\varepsilon$ can be derived from the space curve as follows:

$$
\frac{\mathrm{d} \mathbf{r}}{\mathrm{d} s}=(1+\varepsilon) \mathbf{t}
$$

Here, $\mathbf{t}$ is now the unit tangent vector of the deformed rod. The bending deformations $C_{1,2}$ and twisting deformation $M$ are:

$$
C_{1,2}=\frac{\mathrm{d} \mathbf{t}}{\mathrm{d} s} \cdot \mathbf{a}_{1,2}, \quad M=\frac{\mathrm{d} \mathbf{a}_{\mathbf{1}}}{\mathrm{d} s} \cdot \mathbf{a}_{2} .
$$

Since we restrict our model to rods in a two-dimensional plane, only one curvature deformation and the extensional strain remain. The elastic energy 
in the framework of the Kirchhoff theory then is given by

$$
E_{\text {elastic }}=\frac{1}{2} \int_{0}^{l_{f}} \mathrm{~d} s\left(\kappa C^{2}+\mu \varepsilon^{2}\right),
$$

with the material parameters $\kappa$ (the bending rigidity) and $\mu$ (the stretching rigidity.

A cylindrical elastic rod of Radius $r$, length $l_{f}^{0}$ and Young's modulus $Y$ exhibits a restoring force of

$$
F=\frac{Y \pi r^{2}}{2 l_{f}^{0}} \Delta l,
$$

when stretched by $\Delta l$. The stretching rigidity $\mu$ of such a rod therefore reads

$$
\mu=Y \pi r^{2}
$$

The bending moment of a circular rod is calculated from its second moment of area; this leads to the following bending rigidity $\kappa$ :

$$
\kappa=\frac{1}{4} \pi r^{4} Y
$$

With these expressions, one can for example calculate the equilibrium shape of a rod under load, by using variational calculus to find the minimum-energy form of $\mathbf{r}(s)$. Kirchhoff noted that this leads to the same Euler-Lagrange equations as those for the minimum action of a rigid body in motion. This is known as the Kirchhoff analogy.

Since we intend to simulate the dynamics of elastic rods in a computer, however, we will need to discretize these equations and derive the interactions between the discretized objects, in order to formulate their Newtonian equations of motion.

\subsubsection{Discretization}

For the computer simulations, a finite difference approach is used to discretize the integrals over the center line of the fibers. Here, a fiber is seen as a string 


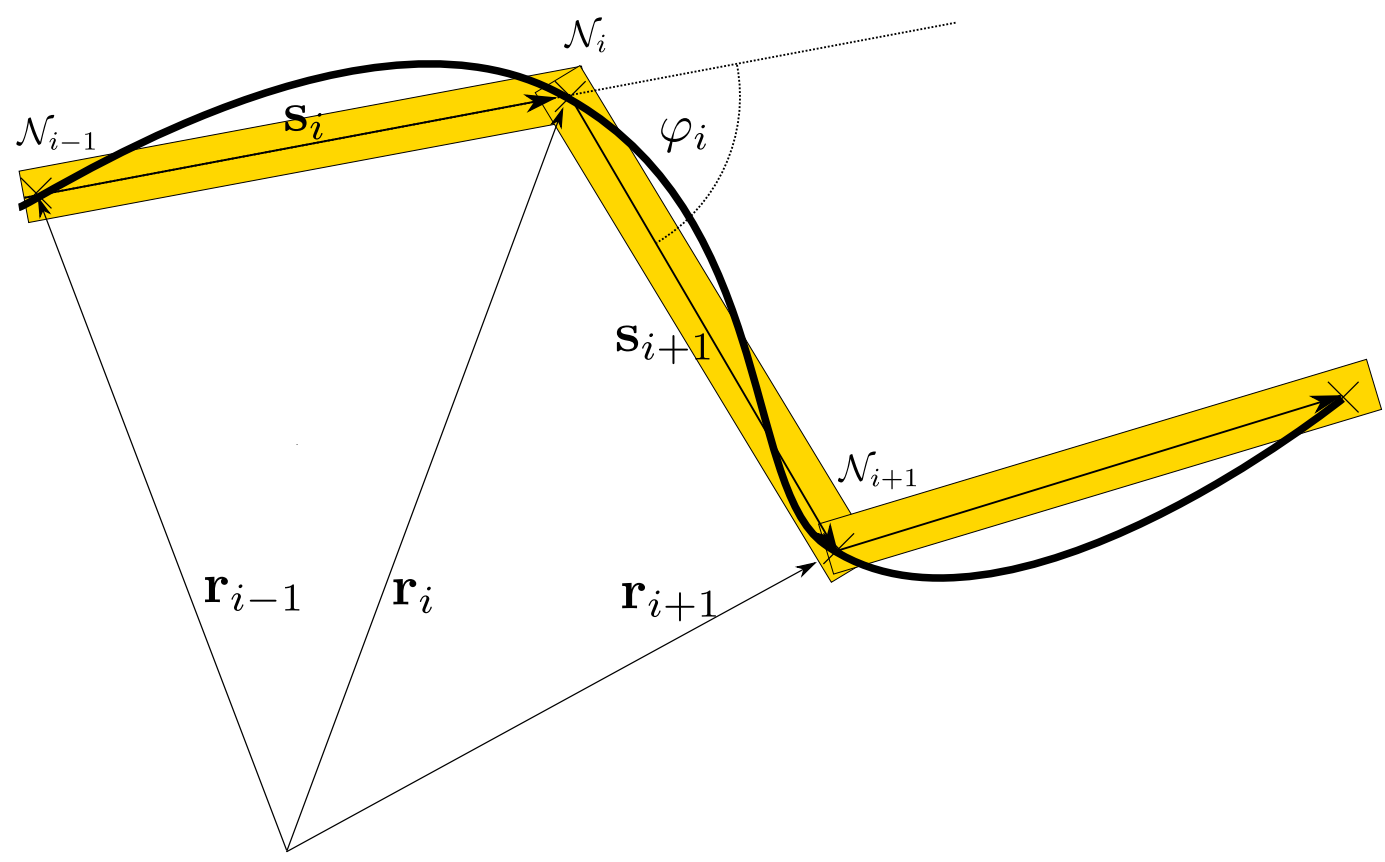

Figure 4.1: A discretized elastic rod is described by the positions $\mathbf{r}_{i}$ of its nodes. Segment vectors are denoted as $\mathbf{s}_{i}$, and bending angles as $\varphi_{i}$.

of segments $\mathcal{S}_{i}$, connected by the nodes $\mathcal{N}_{i}$. The segments are described by vectors $\mathbf{s}_{i}=\left(s_{i}^{x} ; s_{i}^{y}\right)$ of length $l_{i}=\left|\mathbf{s}_{i}\right|$, connecting nodes at positions $\mathbf{r}_{i}$. The values corresponding to the undeformed state are denoted by an upper index of 0 . The angle between two consecutive segments is associated with the corresponding node and is called $\varphi_{i}$. Figure 4.1 illustrates the discretization.

In the simulations, we will discretize each fiber by 10 segments. These segments will then further be split in two at those points where we wish to introduce a fixed link to another fiber. The new node generated within the split segment is then coupled to the corresponding new node of the crossing segment, which was also split. In this way, we implement fixed links as introduced in section 3.2 .

This means that the length of an undeformed segment is not known a priori, although typically it will be close to the length of a segment without crosslinks, which we will call $l_{s}$.

The elastic properties of a continuous fiber are mapped onto the discrete elements (the segments and nodes) in the fashion of a bead-spring-model, like 
it is used in simulations of polymers: The segments are viewed as massless extensional springs connecting the nodes, while the nodes carry the mass of the fiber and are attached to the segments by torsional springs. This approach is similar to that used by Ning and Melrose to simulate flexible fibers [NM99.

The extensional strain $\varepsilon_{i}$ of a segment $\mathcal{S}_{i}$ is then simply the relative length difference of a segment from its undeformed state:

$$
\varepsilon_{i}=\left(\frac{l_{i}-l_{i}^{0}}{l_{i}^{0}}\right)
$$

Since each segment is straight, it follows that curvature is necessarily associated with the nodes. In the continuous case, we defined the curvature as the derivative of the tangent vector with respect to the arc length. Moving from derivatives to finite differences, the discrete curvature at node $\mathcal{N}_{i}$ is:

$$
C_{i}=\left|\frac{\delta^{2} \mathbf{r}_{i}}{\delta l^{2}}\right|
$$

Taking a central difference approach, this becomes

$$
C_{i}=\frac{1}{\tilde{l}_{i}} \sqrt{2\left(1-\cos \varphi_{i}\right)} \approx \frac{\varphi_{i}}{\tilde{l}_{i}}
$$

The approximation holds for small curvatures and hence small angles $\varphi_{i}$. The effective length $\tilde{l}_{i}=\left(l_{i}+l_{i+1}\right) / 2$ is the fiber length associated with the node.

Now the bending and stretching energy integrals from equation (4.3) are replaced with sums as follows:

$$
\begin{aligned}
E_{\text {stretch }} & =\sum_{\mathcal{S}_{i}} \frac{Y \pi r^{2}}{2} \varepsilon_{i}^{2} \\
E_{\text {bend }} & =\sum_{\mathcal{N}_{i}} \frac{Y \pi r^{4}}{8} C_{i}^{2}
\end{aligned}
$$

In this way, bending and stretching energies are assigned to nodes and segments of a discretized fiber, respectively. The equations show that the typical scales of the stretching and bending energies, of fibers deformed by unit extension or unit curvature, are related by the squared aspect ratio:

$$
\mathcal{O}\left(E_{\text {stretch }}\right) \propto \mathcal{O}\left(E_{\text {bend }}\right) \frac{l^{2}}{r^{2}} .
$$




\subsubsection{Dimensionless Variables}

It is always advisable to use dimensionless variables in computer simulations, since numerical accuracy of floating point numbers is best when all the values are of order one. Therefore, we introduce the following dimensionless variables:

- lengths are measured in terms of the length $l_{s}$ of a segment without crosslinks, i.e., one tenth of a fiber length: $l^{\prime}=l / l_{s}$.

This ensures that a segment will typically be of length 1 .

- masses are measured in the units of the mass of a segment of unit length: $m^{\prime}=m / \pi r^{2} l_{s} \rho$, where $\rho$ is the mass density of the fiber material.

- energy is measured in terms of the bending energy of a fiber of unit length and unit curvature: $E^{\prime}=E \cdot 8 l_{s} / Y \pi r^{4}$.

- dimensionless time, consequently, is measured in units of $t^{\prime}=t / \sqrt{8 \rho a^{4} / Y \pi}$.

Another dimensionless parameter is the fiber aspect ratio

$$
a=\frac{l_{s}}{r},
$$

which we will use instead of a dimensionless radius.

In these units, equations (4.10) and 4.11) become (dropping the primes):

$$
\begin{aligned}
E_{\text {stretch }} & =4 a^{2} \sum_{i} l_{i}^{0} \varepsilon_{i}^{2} \\
E_{\text {bend }} & =\sum_{i} \frac{\varphi_{i}^{2}}{l_{i}^{0}} \approx \sum_{i} \frac{2}{l_{i}^{0}}\left(1-\cos \varphi_{i}\right)
\end{aligned}
$$

Here, the strain is $\varepsilon_{i}=\left(l_{i} / l_{i}^{0}-1\right)$. The use of the cosine to approximate $\varphi^{2}$ in 4.15 avoids the use of an inverse cosine to compute the crossing angle and therefore increases computational speed. It is a valid approximation for small curvatures.

\subsubsection{Elastic Forces}

In order to follow the dynamics of the mechanical system described so far, one has to calculate the elastic stresses.

In the spirit of the well-studied bead-spring model, we view the segments as weightless springs and the nodes between the segments as mass points coupled 


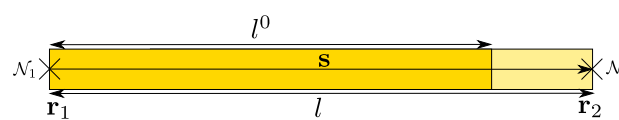

(a) Stretching of a segment of initial length $l^{0}$ to length $l$

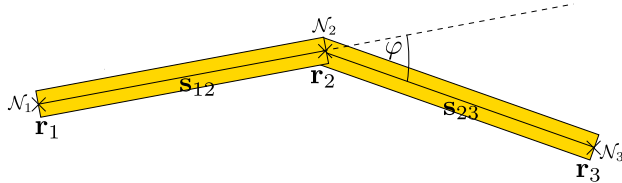

(b) Bending of a fiber around the node $\mathcal{N}_{2}$ by an angle of $\varphi$

Figure 4.2: Schematics of the deformation modes associated with elastic energy costs

to the segments via torsion springs. In this picture, the elastic stresses are expressed as forces on the nodes. Thus, the problem of solving the dynamics of the fibers is equivalent to that of solving Newton's equations of motion for the sum of all elastic forces on the nodes.

These forces are simply the gradients of the potential functions (4.15) and (4.20) with respect to the positions of the nodes.

We refer to fig. 4.2 for an illustration of the vectors and angles employed in this description.

The force a stretched segment exerts on a node is that of a linear spring, i.e. it is proportional to the relative compression $\varepsilon=l_{i}-l_{i}^{0} / l^{0}$ and directed along the segment. This is the force exerted on node $\mathcal{N}_{2}$ in figure 4.2 ;

$$
\mathbf{F}_{2}=-4 a^{2} \frac{\mathbf{s}}{l} \varepsilon
$$

The force on the other node follows from Newton's third law: $\mathbf{F}_{1}=-\mathbf{F}_{2}$.

A bent node (figure 4.2(b) exerts a torque on its adjacent segments. The corresponding force pairs can be calculated by explicitly calculating the derivatives of 4.15 with respect to the node coordinates. The result is best expressed in terms of the segment vectors as follows:

$$
\begin{aligned}
& \mathbf{F}_{1}=\frac{2 l^{0}}{l^{2}}\left(\frac{\cos \varphi}{\left|\mathbf{s}_{12}\right|^{2}} \mathbf{s}_{12}-\frac{1}{\left|\mathbf{s}_{12}\right|\left|\mathbf{s}_{23}\right|} \mathbf{s}_{23}\right) \\
& \mathbf{F}_{2}=\frac{2 l^{0}}{l^{2}}\left[\left(\frac{1}{\left|\mathbf{s}_{12}\right|\left|\mathbf{s}_{23}\right|}+\frac{\cos \varphi}{\left|\mathbf{s}_{23}\right|^{2}}\right) \mathbf{s}_{23}-\left(\frac{1}{\left|\mathbf{s}_{12}\right|\left|\mathbf{s}_{23}\right|}+\frac{\cos \varphi}{\left|\mathbf{s}_{12}\right|^{2}}\right) \mathbf{s}_{12}\right] \\
& \mathbf{F}_{3}=-\frac{2 l^{0}}{l^{2}}\left(\frac{\cos \varphi}{\left|\mathbf{s}_{23}\right|^{2}} \mathbf{s}_{23}-\frac{1}{\left|\mathbf{s}_{23}\right|\left|\mathbf{s}_{12}\right|} \mathbf{s}_{12}\right)
\end{aligned}
$$

Again, the indices are those depicted in figure 4.2 . 


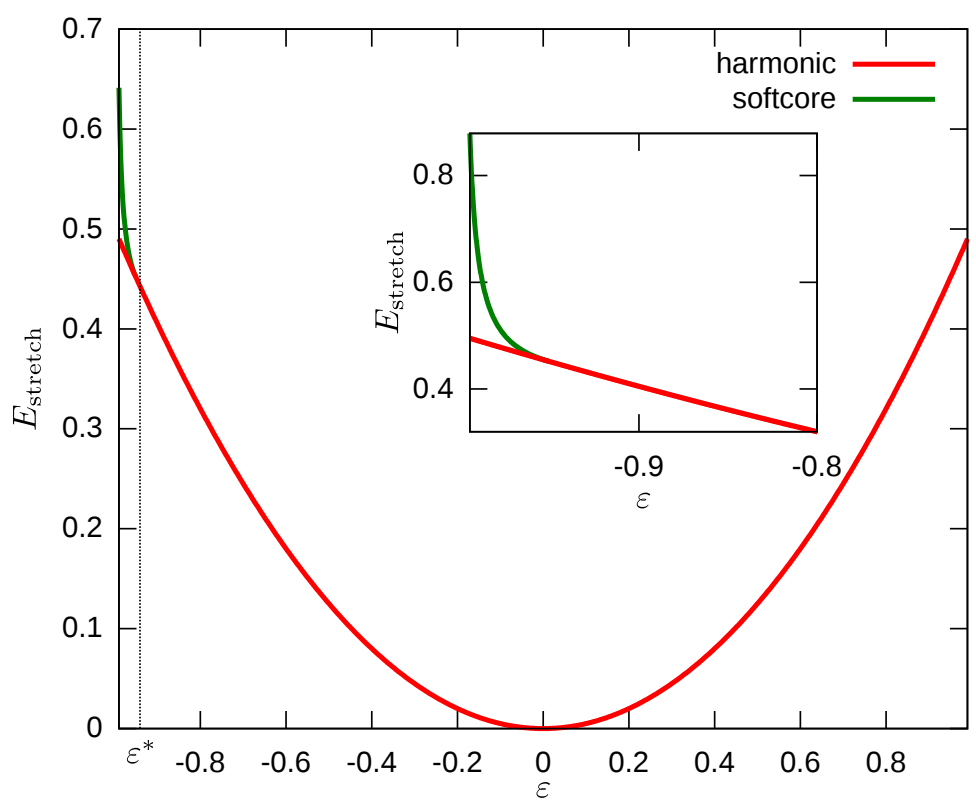

Figure 4.3: Potential $E_{\text {stretch }}$ for segment stretching and compression, plotted as a function of the strain $\varepsilon$, with a softcore repulsion part for extremely compressed segments. The inset is a zoom into the softcore region.

\subsubsection{Softcore Repulsion}

Numerical experiments have shown that in very occasional cases, single segments can become extremely compressed, since the simple harmonic potential allows arbitrary compressions at a finite force. This compression leads to extreme forces due to singularities in some torque force terms for $l / l^{0} \rightarrow 0$, as will be seen in section 4.2.3. This leads to numerical instabilities. In order to avoid these, we introduce a piecewise potential function for segment stretching that amends the harmonic part (4.14) with a softcore repulsion part that diverges for $\varepsilon \rightarrow-1$. With this modification, the stretching energy per segment reads:

$$
E_{\text {stretch }}= \begin{cases}-\frac{l^{0}}{2}\left(\varepsilon^{*}\left(1+\varepsilon^{*}\right)^{2} \frac{l^{0}}{l}+\left(\varepsilon^{*}\right)^{2}\right)+\varepsilon^{*}\left(1+\varepsilon^{*}\right) & \frac{l}{l^{0}}<1+\varepsilon^{*} \\ \frac{l^{0}}{2}\left(\frac{l}{l^{0}}-1\right)^{2} & \frac{l}{l^{0}}>1+\varepsilon^{*}\end{cases}
$$

The value $\varepsilon^{*}$ is the maximum compression above which the softcore repulsion sets in. Throughout the whole work we chose $\varepsilon^{*}=-0.95$. Figure 4.3 
illustrates this potential.

The force law (4.16) then also becomes a piecewise function:

$$
\mathbf{F}_{2}=-4 a^{2} \frac{\mathbf{s}}{l} \cdot \begin{cases}\frac{l-l^{0}}{l^{0}} & \text { for } \frac{l-l^{0}}{l^{0}}<\epsilon \\ \epsilon\left(1+\epsilon^{2}\right)\left(\frac{l^{0}}{l}\right)^{2} & \text { for } \frac{l-l^{0}}{l^{0}}>\epsilon\end{cases}
$$

This means that when the segment is compressed below $\varepsilon^{*}$, an extensional force will eventually grow large enough to counteract any compressional forces before the extension reaches zero.

\subsection{Liquid Bridges as Mobile Crosslinks}

Non-crosslinked fiber contacts in a wet system, as they have been described and examined in chapter 2, are fundamentally different from the fixed crosslinks that are used to describe biopolymer networks or entangled fiber networks as introduced in chapter 3. Those crosslinks impose a hard constraint on the translational degrees of freedom of the crossing fibers: The fibers are not allowed to move with respect to each other.

On the other hand, liquid bridges primarily exert torques and do not impede relative motion. In fact, when the crossing fibers are straight, translations of the fibers cannot change the liquid morphology (keeping in mind that we do not consider contact angle hysteresis), and so the interfacial free energy of the bridge is invariant to these motions. The fibers may freely slide along each other. Another way to look at this is to say that the liquid bridge itself is mobile. It can change its position without energetic cost. This means that pinning of the contact line and friction between the fibers are not considered in this model.

This outlines the strategy for incorporating liquid bridges in a discrete element simulation: They constitute a new type of crosslink-like object that is attached to a pair of segments and exerts forces and torques on them. It is also mobile: Its position is determined by the crossing points of the fibers it connects. Furthermore, it can be created and destroyed when contact points appear or disappear in the course of the dynamics of the network, since liquid bridges in equilibrium with a vapor phase in the constant pressure ensemble will also be created by capillary condensation when two fibers approach, and will evaporate when they rupture because their fibers have been pulled apart. 
The specific way in which we track the creation, destruction and motion of liquid bridge objects is described further on, in appendix A.2. The following sections describe our model potential, which is designed to capture the essential physics derived in the numerical results from chapter 2 , and the forces and torques it gives rise to.

\subsubsection{Sliding Contact Bridges}

The wetting liquid is incorporated by detecting and tracking crossing points of fibers and associating each with a potential energy that is a function of the crossing angle $\alpha$. This potential should represent the grand canonical free energy of the interface of the liquid filament that connects the two fibers with a fixed Laplace pressure, as it has been examined in section 2.3 .

A liquid bridge potential should have the following properties:

i) it should capture the power law dependence of the interfacial energy on the crossing angle $\alpha$ that was observed in chapter 2. The bridge energy $E_{\mathrm{b}}$ should scale as $E_{\mathrm{b}} \propto \alpha^{-\eta}$, with $\eta=1$ for the ensemble of constant pressure. Since there are no specially distinguished values of the angle $\alpha$ apart from $\pi / 2$ and a small angle $\alpha_{c}$ close to complete alignment, a power law behavior is also what one expects in this intermediate region.

ii) since the discrete element model deals with segments of finite length, it should nevertheless not diverge for $\alpha \rightarrow 0$. Instead, the capillary energy associated with the case of $\alpha=0$ should represent the wetting energy of two cylinders of finite length that are completely aligned. This determines the behavior below the angle $\alpha_{c}$ which determines the point of contact of the finite segments.

iii) The potential should capture the correct symmetry at $\alpha=\pi$

With this in mind, we propose the following function as our liquid bridge potential:

$$
E_{\mathrm{b}}=\beta\left(c+c^{2}\right)^{\eta / 2}\left(\frac{1}{c}-\frac{1}{c+\sin ^{2} \alpha}\right)^{\eta / 2}-\beta
$$

Here, $\beta$ is the non-dimensional difference in wetting energies between a bridge at $\alpha=\frac{\pi}{2}$ and a fully aligned bridge, $c$ is a small number governing the 


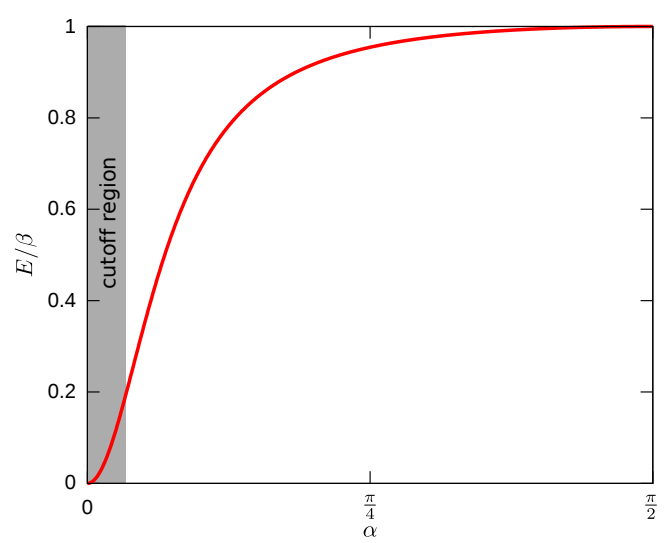

(a) Energy

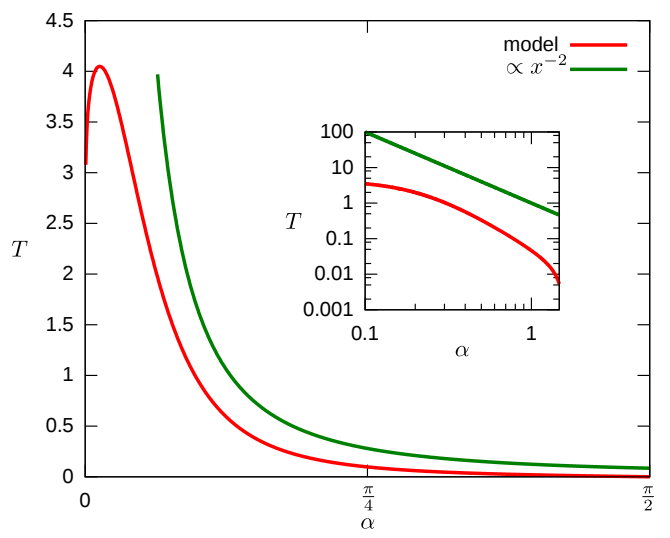

(b) Torque

Figure 4.4: Model potential of the capillary interaction 4.22 for $\eta=1$ and $c=0.05$. (a) Energy as a function of crossing angle; (b) Capillary torque, with a guide to the eye to illustrate the power-law behavior away from the cutoff region. The inset in (b) shows the scaling behavior as a log-log plot.

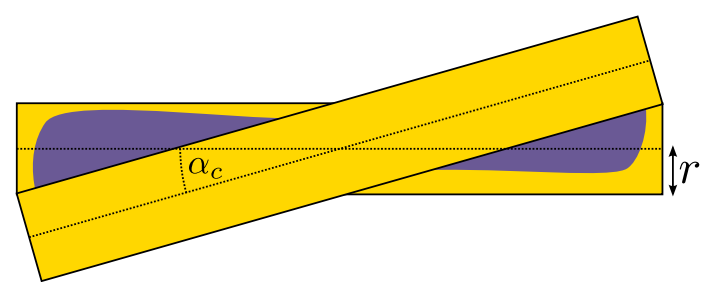

Figure 4.5: The limit case of small crossing angles

small angle cutoff of the capillary interaction, and $\eta$ is the power law exponent. The motivations behind choosing these parameters will be explained in the following.

The functional form of this model potential is plotted in figure 4.4.

When a liquid bridge is formed between two crossing segments, it should model the effects of a wetting liquid forming a filament between these parts of the fibers. Since it has been established in chapter 2 that the wetting energy depends largely on the wetted length, it is clear that the power law behavior ceases to be valid in a discretized model once the full lengths of these two segments are wet. Instead, once this is the case and the two segments 


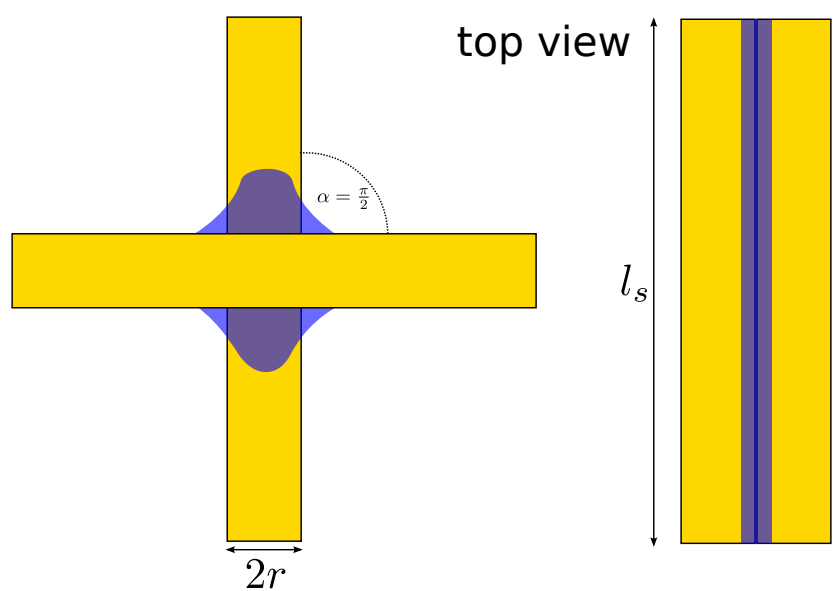

side view
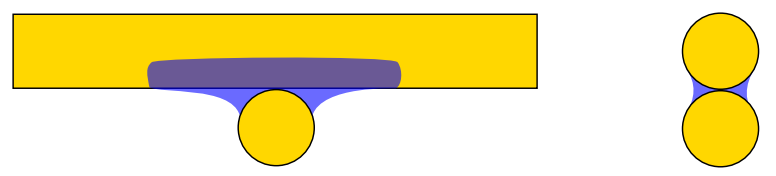

Figure 4.6: Sketch of two crossing segments exhibiting minimal (left) and maximal (right) wetted surface area

are completely aligned, a new liquid bridge will form between the adjacent segments which have now come into contact. In this way, liquid filaments are discretized as sets of liquid bridge objects. A single bridge object must have a finite energy once the segments are completely aligned.

The model potential 4.22 takes care of this finite angle cutoff by the use of the parameter $c$ : Once $\sin ^{2} \alpha \approx c$, the angular dependence of 4.22 becomes insignificant, and a constant cutoff value takes over. Figure 4.5 shows that the limiting wetting length is reached at the cutoff angle value $\alpha_{c}$, for which:

$$
\sin \alpha_{c}=\frac{2 r}{\left\langle l^{0}\right\rangle} \Rightarrow c=\sin ^{2} \alpha_{c}=\frac{4 r^{2}}{\left\langle l^{0}\right\rangle^{2}}
$$

Here the average segment length $\left\langle l^{0}\right\rangle$ has been considered as the reference value.

Since energies are measured in terms of the bending energy, $\beta$ can be seen as a ratio between wetting energies (as governed by surface tension) and elastic energies (as governed by the elastic modulus of the fibers).

To quantitatively understand the parameter $\beta$, one has to look at the typical wetting energies of two crossing segments. These vary between a maximum 
value $\hat{E}_{\mathrm{b}}^{\mathrm{max}}$ for aligned segments and a minimum $\hat{E}_{\mathrm{b}}^{\mathrm{min}}$ for perpendicular ones (see figure 4.6). The wetting energy is dominated by the solid-liquid contact area $A_{s l}$, and can hence be estimated by:

$$
\hat{E}_{\mathrm{b}} \approx \sigma_{l v} \cos \theta A_{s l} .
$$

Since this area varies between approx. $r^{2}$ and $r l_{s}$ for the two cases depicted in figure 4.6, and since $l_{s} \gg r$, one should choose $\beta$ as something like

$$
\beta=\frac{8 \sigma_{l v} \cos \theta l_{s}^{2}}{\pi r^{3} Y}
$$

\subsubsection{Effective Crossing Angles}

In a bent fiber, the tangential orientation varies continuously along the length of the fiber. Taking the discretization approach detailed above literally, crossing angles would jump discontinuously between straight segments, while staying constant along a segment. Thus, liquid crossings cannot take all curvature effects into account, since the curvature is seen as localized in the nodes.

To compensate for this, and to avoid discontinuities in the bridge energies, the crossing angle between two segments has to be calculated from an interpolated version of the fiber orientations. We choose a linear interpolation of the discrete orientation angles $\Phi_{i}=\arctan \left(s_{i}^{y} / s_{i}^{x}\right)$ of the segments in the Cartesian plane, such that the interpolated angle acquires a value of $\Phi_{i}$ at the midpoint of segment $\mathcal{S}_{i}$, and a value of $\left(\Phi_{i}+\Phi_{j}\right) / 2$ at the node to neighboring segments $\mathcal{S}_{j}$ :

$$
\begin{aligned}
\tilde{\Phi}(t) & = \begin{cases}\frac{\Phi_{i-1}+\Phi_{i}}{2}+t\left(\Phi_{i}-\Phi_{i-1}\right) & t<\frac{1}{2} \\
\frac{\Phi_{i}+\Phi_{i+1}}{2}+(1-t)\left(\Phi_{i}-\Phi_{i+1}\right) & t \geq \frac{1}{2}\end{cases} \\
t & =\frac{s}{l_{i}}
\end{aligned}
$$

for a point on segment $\mathcal{S}_{i}$ that is a distance $t \cdot l_{i}$ away from the segment's first node. The angle on the last half of a segment with no neighbor is considered to be constant, i.e., the fiber end points always have zero curvature.

When the distances $s_{a}$ and $s_{b}$ of a crossing point to the nearest nodes on the crossing segments are known, the crossing angle is then simply

$$
\tilde{\alpha}_{a, b}=\tilde{\Phi}_{a}\left(s_{a}\right)-\tilde{\Phi}_{b}\left(s_{b}\right)
$$




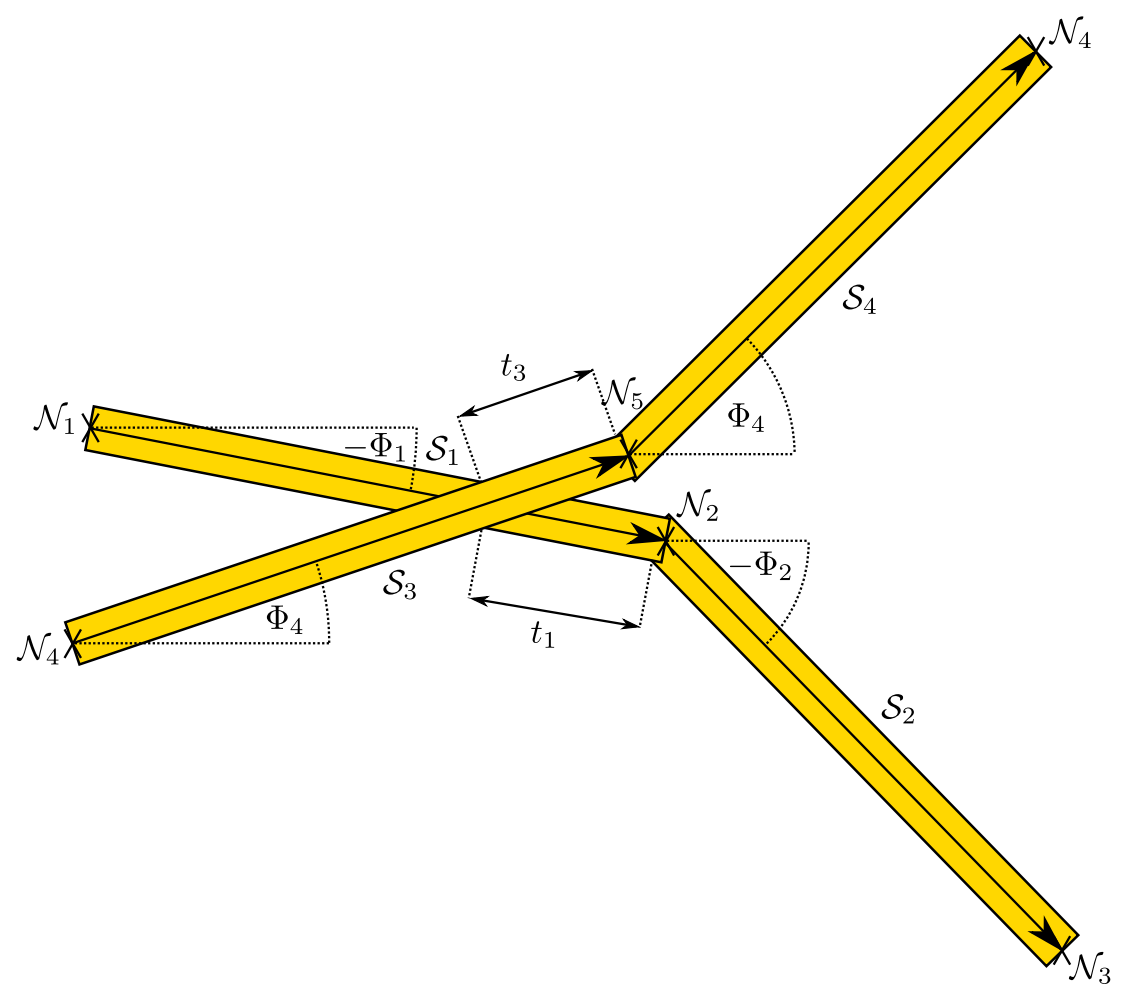

Figure 4.7: Schematic of all nodes influencing the crossing angle of two fibers.

Using this crossing angle enables the liquid bridge to feel the effects of curvature, even though it is strictly just at the intersection of two straight segments.

\subsubsection{Liquid Forces}

Now it remains to calculate the capillary stresses on the fibers. As before, this is done by calculating the forces, i.e. the potential gradients, on the nodes of the fibers that interact with a liquid bridge.

Figure 4.7 depicts two crossing segments and their respective nearest neighbors. The term nearest neighbor of a segment $\mathcal{S}_{i}$ in the context of liquid bridges should be taken to mean that segment attached to $\mathcal{S}_{i}$ that is closest to the bridge. In this sense, a segment can have either zero or one nearest neighbors. The interpolated crossing angle $\tilde{\alpha}$ depends on the positions of all six depicted nodes. Thus, this system of four segments is the minimal subsystem associated with a liquid bridge. Because the coordinates of all six nodes 
enter the calculation of $\tilde{\alpha}$, a liquid bridge at this crossing will exert a force on each of these nodes.

Please note that figure 4.7 uses the convention that the segment vectors point towards the neighboring segments that are nearest to the bridge. The orientation angles are measured accordingly. Also, the arc length parameters $t_{1}$ and $t_{3}$ follow the convention depicted, i.e., they are measured between the crossing point and the node towards the nearest neighbor. All these conventions are without loss of generality, although they have to be accounted for in the simulations.

In the following, we name the coordinates of the six nodes

$$
x_{i}=\left\{r_{1}^{x}, r_{1}^{y}, \ldots, r_{6}^{x}, r_{6}^{y}\right\} \text {. }
$$

The forces can then be calculated as the gradients of the liquid energy

$$
E_{\mathrm{b}}\left(\tilde{\alpha}\left(x_{1}, \ldots, x_{12}\right)\right)
$$

given by equation 4.22 with respect to each node position, while all other node positions are kept constant.

It proves advantageous to calculate these gradients not in Cartesian space but rather in the space of a set of generalized coordinates. The subsystem has twelve degrees of freedom (the two-dimensional coordinates of the six nodes $x_{i}$ ), which can be transformed into the following new coordinates:

- The lengths $l_{1}, l_{2}, l_{3}, l_{4}$ of the segments

- The non-dimensionalized parameters $t_{1}$ and $t_{3}$, corresponding to the distances of the connecting nodes to the crossing points on segments 1 and 3, normalized by the respective segment lengths.

- The orientation angles $\Phi_{1}, \Phi_{2}, \Phi_{3}, \Phi_{4}$ of the segments in the Cartesian plane

- The two Cartesian coordinates $\left(x_{b}, y_{b}\right)$ of the liquid bridge (i.e., the crossing point).

These coordinates are the natural ones to define the interpolated angle $\tilde{\alpha}$, as it was done in equation 4.28. Corresponding to these coordinates are the principal modes of motion by which $\tilde{\alpha}$ can be changed: Rotations of the four segments (corresponding to changes in the $\Phi_{i}$ ) and parallel shifts of the 
fibers along each other (corresponding to changes in the $t_{i}$ ). The remaining generalized coordinates are associated with floppy modes that do not change the liquid morphology: Stretching of the segments around the crossing point (i.e., changing the $l_{i}$ independently), and a translation of the subsystem as a whole (i.e., changing $x_{b}$ or $y_{b}$ ).

These twelve new coordinates will be denoted $\xi_{i}$ in the order they appear in the above list. The transformation function $f: \xi_{i} \rightarrow r_{i}$ is then derived by elementary geometry as:

$$
\begin{aligned}
r_{1}^{x} & =-l_{1} \cos \Phi_{1}\left(1-t_{1}\right)+x_{b} \\
r_{1}^{y} & =-l_{1} \sin \Phi_{1}\left(1-t_{1}\right)+y_{b} \\
r_{2}^{x} & =l_{1} t_{1} \cos \Phi_{1}+x_{b} \\
r_{2}^{y} & =l_{1} t_{1} \sin \Phi_{1}+y_{b} \\
r_{3}^{x} & =l_{1} t_{1} \cos \Phi_{1}+l_{2} \cos \Phi_{2}+x_{b} \\
r_{3}^{y} & =l_{1} t_{1} \sin \Phi_{1}+l_{2} \sin \Phi_{2}+y_{b} \\
r_{4}^{x} & =-l_{3} \cos \Phi_{3}\left(3-t_{3}\right)+x_{b} \\
r_{4}^{y} & =-l_{3} \sin \Phi_{3}\left(3-t_{3}\right)+y_{b} \\
r_{5}^{x} & =l_{3} t_{3} \cos \Phi_{3}+x_{b} \\
r_{5}^{y} & =l_{3} t_{3} \sin \Phi_{3}+y_{b} \\
r_{6}^{x} & =l_{3} t_{3} \cos \Phi_{3}+l_{4} \cos \Phi_{4}+x_{b} \\
r_{6}^{y} & =l_{3} t_{3} \sin \Phi_{3}+l_{4} \sin \Phi_{4}+y_{b}
\end{aligned}
$$

To calculate the Cartesian gradient of equation (4.22) with $\alpha$ substituted by the interpolated $\tilde{\alpha}$ as defined in (4.28), we use the chain rule to get

$$
\frac{\partial E(\tilde{\alpha})}{\partial r_{i}}=\frac{\partial E(\tilde{\alpha})}{\partial \tilde{\alpha}} \sum_{j=1}^{12} \frac{\partial \tilde{\alpha}}{\partial \xi_{j}} \frac{\partial \xi_{j}}{\partial r_{i}}
$$

The first derivative in the sum of equation (4.30) can be seen as the gradient of $\tilde{\alpha}$ in the new coordinate system. Then, the operation is a multiplication of this gradient with the matrix

$$
J^{-T}=\frac{\partial f^{-} 1\left(x i_{1}, \ldots, x i_{1} 2\right)^{T}}{\partial\left(r_{1}, \ldots, r_{1} 2\right)} .
$$

This is the transpose of the Jacobian of the inverse of function $f$. It can be calculated most easily by calculating the Jacobian of $f$ and then inverting it, i.e., by using the inverse function theorem. 
This gives: $J^{-1}=$

$$
\begin{aligned}
& \left(\begin{array}{cccccc}
-\cos \Phi_{1} & -\sin \Phi_{1} & \cos \Phi_{1} & \sin \Phi_{1} & 0 & 0 \\
0 & 0 & -\cos \Phi_{2} & -\sin \Phi_{2} & \cos \Phi_{2} & \sin \Phi_{2} \\
0 & 0 & 0 & 0 & 0 & 0 \\
0 & 0 & 0 & 0 & 0 & 0 \\
\frac{t_{1} \sin \Phi_{3}}{l_{1} \sin \alpha} & -\frac{t_{1} \cos \Phi_{3}}{l_{1} \sin \alpha} & -\frac{\left(t_{1}-1\right) \sin \Phi_{3}}{l_{1} \sin \alpha} & \frac{\left(t_{1}-1\right) \cos \Phi_{3}}{l_{1} \sin \alpha} & 0 & 0 \\
\frac{t_{1} \sin \Phi_{1}}{l_{3} \sin \alpha} & -\frac{t_{1} \cos \Phi_{1}}{l_{3} \sin \alpha} & -\frac{\left(t_{1}-1\right) \sin \Phi_{1}}{l_{3} \sin \alpha} & \frac{\left(t_{1}-1\right) \cos \Phi_{1}}{l_{3} \sin \alpha} & 0 & 0 \\
\frac{\sin \Phi_{1}}{l_{1}} & -\frac{\cos \Phi_{1}}{l_{1}} & -\frac{\sin \Phi_{1}}{l_{1}} & \frac{\cos \Phi_{1}}{l_{1}} & 0 & 0 \\
0 & 0 & \frac{\sin \Phi_{2}}{l_{2}} & -\frac{\cos \Phi_{2}}{l_{2}} & -\frac{\sin \Phi_{2}}{l_{2}} & \frac{\cos \Phi_{2}}{l_{2}} \\
0 & 0 & 0 & 0 & 0 & 0 \\
0 & 0 & 0 & 0 & 0 & 0 \\
-\frac{t_{1} \cos \Phi_{3} \sin \Phi_{1}}{\sin \alpha} & \frac{t_{1} \cos \Phi_{3} \cos \Phi_{1}}{\sin \alpha} & \frac{\left(t_{1}-1\right) \cos \Phi_{3} \sin \Phi_{1}}{\sin \alpha} & -\frac{\left(t_{1}-1\right) \cos \Phi_{3} \cos \Phi_{1}}{\sin \alpha} & 0 & 0 \\
-\frac{t_{1} \sin \Phi_{3} \sin \Phi_{1}}{\sin \alpha} & \frac{t_{1} \sin \Phi_{3} \cos \Phi_{1}}{\sin \alpha} & \frac{\left(t_{1}-1\right) \sin \Phi_{3} \sin \Phi_{1}}{\sin \alpha} & -\frac{\left(t_{1}-1\right) \sin \Phi_{3} \cos \Phi_{1}}{\sin \alpha} & 0 & 0
\end{array}\right. \\
& \left.\begin{array}{cccccc}
0 & 0 & 0 & 0 & 0 & 0 \\
0 & 0 & 0 & 0 & 0 & 0 \\
-\cos \Phi_{3} & -\sin \Phi_{3} & \cos \Phi_{3} & \sin \Phi_{3} & 0 & 0 \\
0 & 0 & -\cos \Phi_{4} & -\sin \Phi_{4} & \cos \Phi_{4} & \sin \Phi_{4} \\
-\frac{t_{3} \sin \Phi_{3}}{l_{1} \sin \alpha} & \frac{t_{3} \cos \Phi_{3}}{l_{1} \sin \alpha} & \frac{\left(t_{3}-1\right) \sin \Phi_{3}}{l_{1} \sin \alpha} & -\frac{\left(t_{3}-1\right) \cos \Phi_{3}}{l_{1} \sin \alpha} & 0 & 0 \\
-\frac{t_{3} \sin \Phi_{1}}{l_{3} \sin \alpha} & \frac{t_{3} \cos \Phi_{1}}{l_{3} \sin \alpha} & \frac{\left(t_{3}-1\right) \sin \Phi_{1}}{l_{3} \sin \alpha} & -\frac{\left(t_{3}-1\right) \cos \Phi_{1}}{l_{3} \sin \alpha} & 0 & 0 \\
0 & 0 & 0 & 0 & 0 & 0 \\
0 & 0 & 0 & 0 & 0 & 0 \\
\frac{\sin \Phi_{3}}{l_{3}} & -\frac{\cos \Phi_{3}}{l_{3}} & -\frac{\sin \Phi_{3}}{l_{3}} & \frac{\cos \Phi_{3}}{l_{3}} & 0 & 0 \\
0 & 0 & \frac{\sin \Phi_{4}}{l_{4}} & -\frac{\cos \Phi_{4}}{l_{4}} & -\frac{\sin \Phi_{4}}{l_{4}} & \frac{\cos \Phi_{4}}{l_{4}} \\
\frac{t_{3} \sin \Phi_{3} \cos \Phi_{1}}{\sin \alpha} & -\frac{t_{3} \cos \Phi_{3} \cos \Phi_{1}}{\sin \alpha} & -\frac{\left(t_{3}-1\right) \sin \Phi_{3} \cos \Phi_{1}}{\sin \alpha} & \frac{\left(t_{3}-1\right) \cos \Phi_{3} \cos \Phi_{1}}{\sin \alpha} & 0 & 0 \\
\frac{t_{3} \sin \Phi_{3} \sin \Phi_{1}}{\sin \alpha} & -\frac{t_{3} \cos \Phi_{3} \sin \Phi_{1}}{\sin \alpha} & -\frac{\left(t_{3}-1\right) \sin \Phi_{3} \sin \Phi_{1}}{\sin \alpha} & \frac{\left(t_{3}-1\right) \cos \Phi_{3} \sin \Phi_{1}}{\sin \alpha} & 0 & 0
\end{array}\right)
\end{aligned}
$$

The non-interpolated crossing angle $\alpha$ appearing here is to be read as $\alpha=$ $\Phi_{3}-\Phi_{1}$, where the angles are measured according to the convention depicted in figure 4.7 .

The rows of this matrix are the gradients of the new coordinates $\xi_{i}$ in Cartesian space. Using this matrix one can transform the energy gradient in $\xi$ space into the Cartesian gradient. The gradient in $\xi$-space is easily calculated 
from equation 4.27) and 4.28):

$\frac{\partial \tilde{\alpha}}{\partial \xi_{i}}=\left(0,0,0,0,-\left(\Phi_{1}-\Phi_{2}\right), \Phi_{3}-\Phi_{4},-\left(t_{1}+\frac{1}{2}\right), t_{1}-\frac{1}{2}, t_{3}+\frac{1}{2},-t_{3}+\frac{1}{2}, 0,0\right)$

The components of the Cartesian gradient of $\tilde{\alpha}$ are the gradients of the interpolated crossing angle with respect to the displacements of the six nodes. Thus, it is convenient to split that vector into six $\mathbb{R}^{2}$-vectors. Multiplying these with the common prefactor

$$
A:=-\frac{\partial E(\tilde{\alpha})}{\partial \tilde{\alpha}}=-\frac{\left(c+c^{2}\right)^{\eta / 2} \eta \cos \tilde{\alpha} \sin \tilde{\alpha}\left(\frac{1}{c}-\frac{1}{c+\sin ^{2} \tilde{\alpha}}\right)^{\eta / 2-1}}{\left(c+\sin ^{2} \tilde{\alpha}\right)^{2}}
$$

gives, by the chain rule, the forces on the respective nodes:

$$
\begin{aligned}
& \mathbf{F}_{1}= A\left[\left(\Phi_{1}-\Phi_{2}\right) \frac{t_{1}}{l_{1} \sin \alpha}\left(\begin{array}{c}
-\sin \Phi_{3} \\
\cos \Phi_{3}
\end{array}\right)+\left(\Phi_{3}-\Phi_{4}\right) \frac{t_{1}}{l_{3} \sin \alpha}\left(\begin{array}{c}
\sin \Phi_{1} \\
-\cos \Phi_{1}
\end{array}\right)\right. \\
&\left.+\left(t_{1}+\frac{1}{2}\right) \frac{1}{l_{1}}\left(\begin{array}{c}
-\sin \Phi_{1} \\
\cos \Phi_{1}
\end{array}\right)\right] \\
& \mathbf{F}_{2}=A {\left[\left(\Phi_{1}-\Phi_{2}\right) \frac{\left(t_{1}-1\right)}{l_{1} \sin \alpha}\left(\begin{array}{c}
\sin \Phi_{3} \\
-\cos \Phi_{3}
\end{array}\right)+\left(\Phi_{3}-\Phi_{4}\right) \frac{\left(t_{1}-1\right)}{l_{3} \sin \alpha}\left(\begin{array}{c}
-\sin \Phi_{1} \\
\cos \Phi_{1}
\end{array}\right)\right.} \\
&\left.+\left(t_{1}+\frac{1}{2}\right) \frac{1}{l_{1}}\left(\begin{array}{c}
-\sin \Phi_{1} \\
-\cos \Phi_{1}
\end{array}\right)+\left(t_{1}-\frac{1}{2}\right) \frac{1}{l_{2}}\left(\begin{array}{c}
\sin \Phi_{2} \\
-\cos \Phi_{2}
\end{array}\right)\right] \\
& \mathbf{F}_{3}= A\left(t_{1}-\frac{1}{2}\right) \frac{1}{l_{2}}\left(\begin{array}{c}
-\sin \Phi_{2} \\
\cos \Phi_{2}
\end{array}\right) \\
& \mathbf{F}_{4}= A\left[\left(\Phi_{1}-\Phi_{2}\right) \frac{t_{3}}{l_{1} \sin \alpha}\left(\begin{array}{c}
-\sin \Phi_{3} \\
-\cos \Phi_{3}
\end{array}\right)+\left(\Phi_{3}-\Phi_{4}\right) \frac{t_{3}}{l_{3} \sin \alpha}\left(\begin{array}{c}
-\sin \Phi_{1} \\
\cos \Phi_{1}
\end{array}\right)\right. \\
&\left.+\left(t_{3}+\frac{1}{2}\right) \frac{1}{l_{3}}\left(\begin{array}{c}
-\sin \Phi_{3} \\
-\cos \Phi_{3}
\end{array}\right)\right] \\
& \mathbf{F}_{5}= A\left[\left(\Phi_{1}-\Phi_{2}\right) \frac{\left(t_{3}-1\right)}{l_{1} \sin \alpha}\left(\begin{array}{c}
-\sin \Phi_{3} \\
\cos \Phi_{3}
\end{array}\right)+\left(\Phi_{3}-\Phi_{4}\right) \frac{\left(t_{3}-1\right)}{l_{3} \sin \alpha}\left(\begin{array}{c}
\sin \Phi_{1} \\
-\cos \Phi_{1}
\end{array}\right)\right. \\
&\left.+\left(t_{3}+\frac{1}{2}\right) \frac{1}{l_{3}}\left(\begin{array}{c}
-\sin \Phi_{3} \\
\cos \Phi_{3}
\end{array}\right)+\left(t_{3}-\frac{1}{2}\right) \frac{1}{l_{4}}\left(\begin{array}{c}
-\sin \Phi_{4} \\
\cos \Phi_{4}
\end{array}\right)\right] \\
& \mathbf{F}_{6}= A\left(t_{3}-\frac{1}{2}\right) \frac{1}{l_{4}}\left(\begin{array}{c}
-\sin \Phi_{4} \\
-\cos \Phi_{4}
\end{array}\right) \\
&(4.35)
\end{aligned}
$$


The forces in equation (4.35) separate into different terms, each of which belongs to one of the principal modes of displacement described by the $\xi_{i}$ Coordinates. Certain modes have no influence on the interpolated angle, and hence on the liquid bridge energy, which is reflected in the zeros in the gradient (4.33). Also, certain nodes do not contribute to some of the modes, which is reflected in the zeros in the matrix 4.32). The ones that remain in 4.35 can be interpreted as torques and forces contributing to rotations of the segments and parallel shifts of the fibers along each other, respectively. This is elaborated in the following paragraphs.

\section{Aligning Torques}

The force terms corresponding to the rotational modes are forces acting in a direction perpendicular to the node's respective segments. A pair of such forces, acting on adjacent nodes and corresponding to the variation of their segments' orientation, constitute a torque on that segment.

In a configuration where each crossing segment has a nearest neighbor, the liquid bridge exerts a torque on all four segments as a whole. This torque is distributed among the segments into separate torques. For example, equation (4.35) shows one rotational force acting on $\mathcal{N}_{1}$ (the last term), two acting on $\mathcal{N}_{2}$ (the last two terms) and one acting on $\mathcal{N}_{3} . \mathcal{N}_{2}$ experiences the torque on segment $\mathcal{S}_{1}$ and $\mathcal{S}_{2}$, while $\mathcal{N}_{1}$ and $\mathcal{N}_{3}$ only experience one torque. The torque force terms act perpendicular to their respective segments, and their magnitude is governed by the position of the crossing point along the fiber (in this case, $\left.t_{1}\right)$.

The prefactors $\left(t_{1}+1 / 2\right)$ and $\left(t_{1}-1 / 2\right)$ constitute a weighting factor that emphasizes the torque of the neighboring segment $\mathcal{S}_{2}$ when the crossing point is closer to this segment, and emphasizes the torque on $\mathcal{S}_{1}$ when it is close to the middle of $\mathcal{S}_{1}$.

The prefactors involving the segment lengths $l_{1}$ and $l_{2}$ take the different levers of the two segments into account, so that the torque on each segment before multiplication with the prefactors involving $t_{i}$ has the same magnitude. This means that the total torque on two neighboring segments always adds up to the same magnitude, governed by $A$, and is exactly compensated by the torque on the segments of the remaining fiber.

It can be easily verified that the torques add to zero. This is to be expected, because the angle $\tilde{\alpha}$, and therefore the energy $E_{\mathrm{b}}(\tilde{\alpha})$, depends only on the differences of orientation angles. Therefore, it is invariant under rotations of 


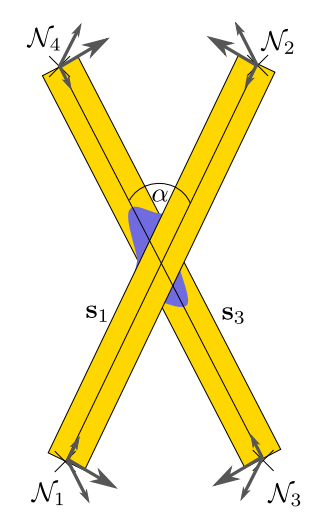

Figure 4.8: Two crossing segments with a liquid bridge

the whole system, so that conservation of angular momentum must apply.

In figure 4.8 , the simpler case of two segments with no nearest neighbors is depicted. Here, the remaining torque force terms form pairs of forces perpendicular to the segments.

\section{Forces}

When the fibers are not straight, they can reduce their crossing angle by sliding along each other. This gives rise to the remaining terms in equation 4.35).

These forces act on the nodes of the crossing segments in such a way that they strive to shift along the directions of their counterparts. Taking the example of segment $\mathcal{S}_{1}$, it is pulled along the direction of $\mathcal{S}_{3}$, corresponding to a change of $t_{3}$, and moving it to a domain where the interpolated orientation of $\mathcal{S}_{3}$ is more aligned with $\mathcal{S}_{1}$. It is also pulled along its own direction, toward the area where its own orientation is more aligned with that of $\mathcal{S}_{3}$.

It is easy to see that those shifting force terms appearing for segment $\mathcal{S}_{3}$ are the counter-forces of those appearing for segment $\mathcal{S}_{1}$ according to Newton's third law.

\section{Modifications for the Case of End-Segments}

When one of the segments does not have a nearest neighbor, its orientation is assumed to be constant with respect to $t$. That is, the end of a fiber is always assumed to be straight in this interpolation model. Because of this, the secondary torque terms and shifting force terms corresponding to the missing neighbor segment in 4.35 vanish. Also, the primary torque forces on the 


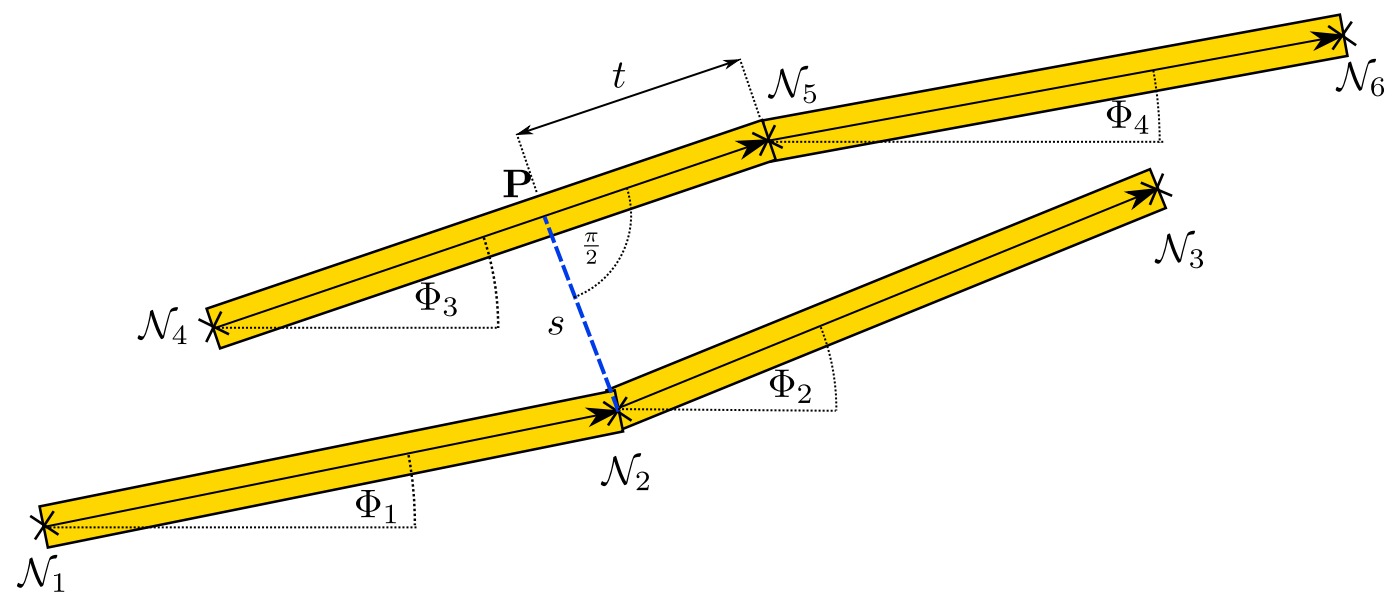

Figure 4.9: Schematic of the subsystem involved in a stretched bridge

end-segment now have a prefactor of 1 instead of $t+1 / 2$, so that the torque on the end-segment alone compensates the torques on the segments of the crossing fiber. This can be verified by explicitly calculating the three-segment case in the same manner as described above.

\subsubsection{Stretched Bridges}

When the motion of the fibers leads to the disappearance of a crossing, i.e., when two segments that previously crossed and formed a liquid bridge slide off each other (and the liquid bridge doesn't just wander to a neighboring segment), the fate of the liquid bridge has to be addressed.

A first approach in this case might be to simply delete the bridge, since loss of contact will in principle revert the fibers back to a non-bridged state with the wetting liquid evaporating. This neglects attractive forces exerted by the bridge stretching between the separated segments, but since in a twodimensional model system these forces are insignificant compared to aligning torques, this seems like a valid approach. However, conservation of energy has to be considered. It turns out, as we will explain in the following, that the omission of the attractive force acting during the short time of the rupture event would violate energy conservation.

In principle, the interfacial free energy of the system need not be continuous: The energy carried by the liquid bridges represents the free energy of the wetting liquid. This can undergo jumps towards lower energy levels whenever 


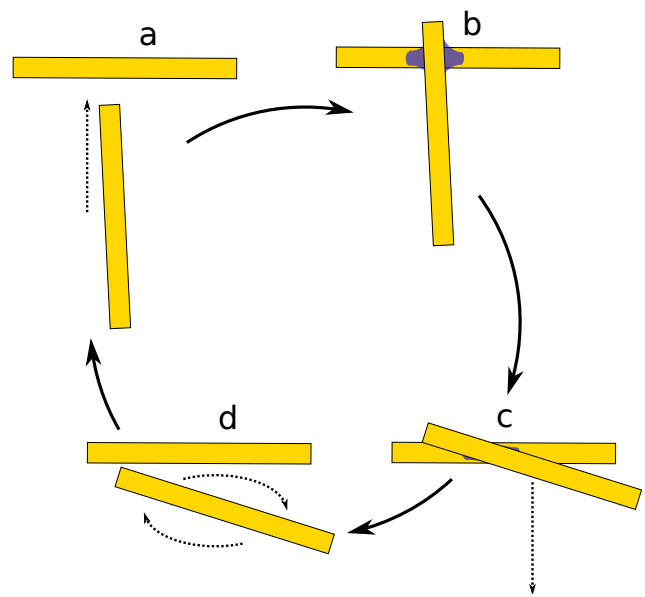

(a)

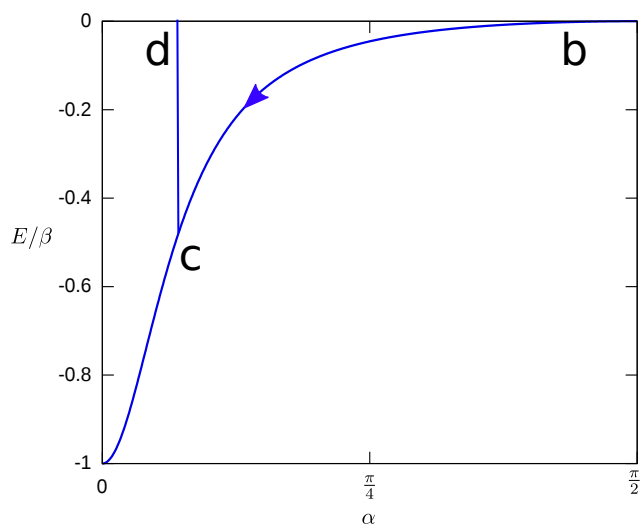

(b)

Figure 4.10: A cyclic process violating energy conservation when stretched bridges are not considered. Figure (b) shows the potential energy ascribed to the liquid bridge in the states depicted on the left. The difference between states $\mathrm{c}$ and $\mathrm{d}$ is the work the bridge has done on the segments, and that is now unaccounted for.

the liquid morphology becomes unstable. An example for an event of this kind is the rupture of a liquid bridge between two spheres. In this picture, the system can be viewed as dissipative: Rupture of a bridge is a dissipative process, because energy is transferred to the internal degrees of freedom of the liquid. In this sense, the liquid provides a dissipative channel. This mechanism of dissipation is widely studied in the area of wet granular systems, where a very similar principle is applied to simulations of spheres with liquid bridges between them (see for example $\left[\mathrm{FGH}^{+} 05\right.$, Her05]). Here, a hysteretic force law captures the bistable nature of the liquid bridge and leads to a mechanism of dissipation that plays a great role in the dynamics of the system.

The dissipation itself occurs by way of damped capillary waves on the liquid that are excited by the rupture event. Therefore, energy can only ever be lost to the liquid system. If a slipped-off bridge is simply deleted, this cannot be guaranteed, since the bridge might have done work on the system before its deletion.

Figure 4.10 depicts a sequence of hypothetical events in which two segments come into contact at $\alpha \approx \pi / 2$ (point $\mathrm{b}$ ). The liquid bridge potential then sets the segments into rotational motion in order to align them (point $\mathrm{c}$ ). When 


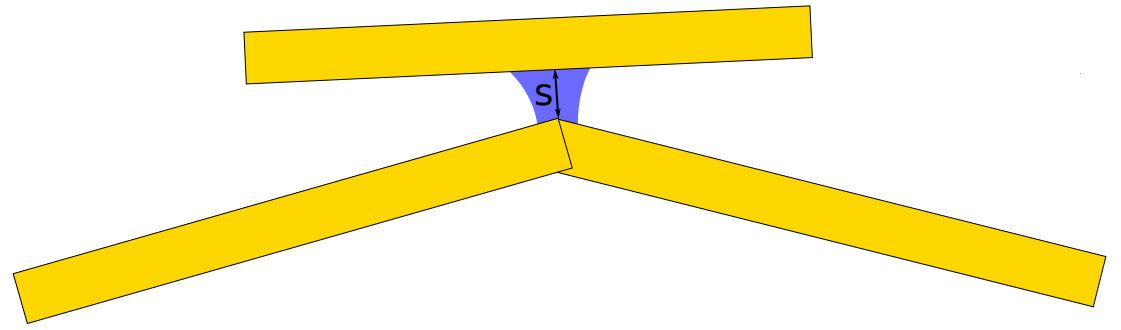

Figure 4.11: A stretched bridge spanned between a node that has lost contact to another fiber and the nearest point on that fiber

the segments lose contact at point $\mathrm{d}$, the liquid bridge vanishes and does not carry energy anymore. Therefore, the internal energy of the system is back in the same state, and the cycle can begin anew, although the system has done work on the segments to align them. This is a perpetuum mobile of the first kind. It can occur only because the information about the work done by capillary forces is lost when the bridges are destroyed. This can only be allowed when the amount of work done is equal to the remaining interfacial energy of the bridge.

Therefore, whenever the two segments associated with a liquid bridge lose contact, one must consider the bridge between the non-intersecting segments as still present, and work has to be done against an attractive force between the segments to rupture the bridge. This work must be at least equal to the amount of interfacial free energy still stored in the bridge.

These events (when a liquid bridge becomes stretched) must be detected and handled in the course of a simulation. Disappearance of a fiber contact occurs in two cases: Either when the end of a fiber glides off another fiber, or when a kink in the fiber slips off a second fiber (see figure 4.11). In the latter case, two bridges disappear at the same time, forming a new stretched bridge.

These cases are handled by introducing a new kind of bridge, called a stretched bridge, that acts over a finite distance. It is stretched between the node that slipped off the crossing fiber and the nearest point on that fiber (see figure 4.11), and is either accounts for one or two contact bridges, depending on the specific case. In addition to the angular dependency introduced before, the capillary energy of a stretched bridge has a dependency on the separation $s$ as well. It is assumed to drop linearly with the separation $s$ until a critical separation $s^{*}$ is reached. At this point, the bridge ruptures. Thus, it exerts a constant attractive force (when the orientations are fixed) 


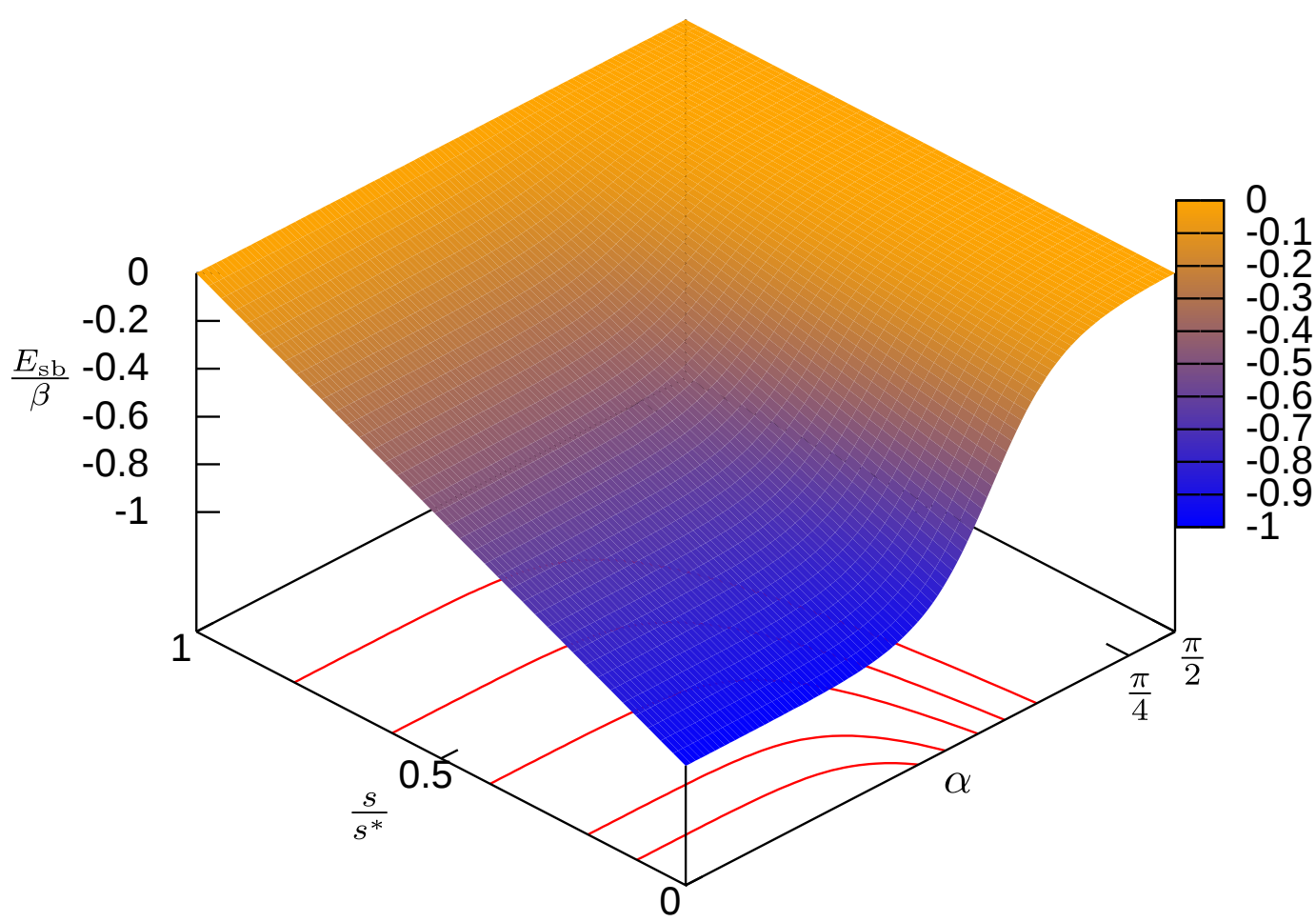

Figure 4.12: Energy of a stretched bridge in the space of angle and separation. Note that $\alpha$ is plotted on a logarithmic scale to make the energy landscape more pronounced. The red lines on the bottom mark lines of constant energy.

from the point where the fibers lose contact until the point of rupture. This aspect of stretched bridges is akin to the minimal capillary model used in simulations of wet systems of spherical grains [Her05], where the model potential as a function of sphere separation is exactly the same.

In order to ensure continuity, the effective angles used for the orientational part of the interaction are taken at the locus of the tethered node and the point of the shortest distance on the fiber, respectively. The model potential for a stretched bridge then takes the form:

$$
E_{\mathrm{sb}}=\left(\beta\left(c+c^{2}\right)^{\eta / 2}\left(\frac{1}{c}-\frac{1}{c+\sin ^{2} \alpha}\right)^{\eta / 2}-\beta\right)\left(1-\frac{s}{s^{*}}\right)
$$

With this model, energy may be lost to the liquid when a bridge forms at angles $\alpha \neq \pi / 2$, but at the point of rupture, the residual energy carried by 
the bridge will always be zero. Figure 4.12 illustrates the energy landscape of this potential.

Figure 4.9 sketches the minimal subsystem influenced by a stretched bridge and again defines the geometrical parameters that serve as generalized coordinates to derive the forces. Here, the point

$$
\mathbf{P}=\left(\begin{array}{c}
x_{a} \\
y_{a}
\end{array}\right)
$$

is the point where the stretched bridge is attached on the second fiber. The generalized coordinates for stretched bridges are then defined as:

$$
\xi_{i}=\left\{l_{1}, l_{2}, l_{3}, l_{4}, t, s, \Phi_{1}, \Phi_{2}, \Phi_{3}, \Phi_{4}, x_{a}, y_{a}\right\}
$$

Since we assume that the stretched bridge always spans the shortest distance between fiber and node, it will always be at a right angle with the segment (the case of a stretched bridge that has slid to the end of a fiber is explained further down). With these parameters, the node positions are calculated as follows:

$$
\begin{aligned}
r_{1} & =s \sin \Phi_{3}-l_{1} \cos \Phi_{1}+x_{a} \\
r_{2} & =-s \cos \Phi_{3}-l_{1} \sin \Phi_{1}+y_{a} \\
r_{3} & =s \sin \Phi_{3}+x_{a} \\
r_{4} & =-s \cos \Phi_{3}+y_{a} \\
r_{5} & =s \sin \Phi_{3}+l_{2} \cos \Phi_{2}+x_{a} \\
r_{6} & =-s \cos \Phi_{3}+l_{2} \sin \Phi_{2}+y_{a} \\
r_{7} & =(1-t) l_{3} \cos \Phi_{3}+x_{a} \\
r_{8} & =(1-t) l_{3} \sin \Phi_{3}+y_{a} \\
r_{9} & =t l_{3} \cos \Phi_{3}+x_{a} \\
r_{10} & =t l_{3} \sin \Phi_{3}+y_{a} \\
r_{11} & =t l_{3} \cos \Phi_{3}+l_{4} \cos \Phi_{4}+x_{a} \\
r_{12} & =t l_{3} \sin \Phi_{3}+l_{4} \sin \Phi_{4}+y_{a}
\end{aligned}
$$

Since the energy stored in a stretched bridge also depends on the separation $s$, the chain rule that has to be used to derive the forces in analogy to 
equation 4.30 is

$$
\frac{\partial E_{\mathrm{sb}}(\tilde{\alpha}, s)}{\partial r_{i}}=\frac{\partial E_{\mathrm{sb}}}{\partial \tilde{\alpha}} \sum_{j=1}^{12} \frac{\partial \tilde{\alpha}}{\partial \xi_{j}} \frac{\partial \xi_{j}}{\partial r_{i}}+\frac{\partial E_{\mathrm{sb}}}{\partial s} \sum_{j=1}^{12} \frac{\partial s}{\partial \xi_{i}} \frac{\partial \xi_{j}}{\partial r_{i}}
$$

The gradients of $\tilde{\alpha}$ and $s$ take the form

$$
\begin{aligned}
\frac{\partial \tilde{\alpha}}{\partial \xi_{i}} & =\left(0,0,0,0, \Phi_{3}-\Phi_{4}, 0,-\frac{1}{2},-\frac{1}{2}, \frac{1}{2}+t, \frac{1}{2}-t\right) \\
\frac{\partial s}{\partial \xi_{i}} & =(0,0,0,0,0,1,0,0,0,0)
\end{aligned}
$$

The transformation matrix reads as follows (see next page): 


$$
J_{\mathrm{sb}}^{-1}=\left(\begin{array}{ccccc}
-\cos \Phi_{1} & -\sin \Phi_{1} & \cos \Phi_{1} & \sin \Phi_{1} & \ldots \\
0 & 0 & -\cos \Phi_{2} & -\sin \Phi_{2} & \ldots \\
0 & 0 & 0 & 0 & \ldots \\
0 & 0 & 0 & 0 & \ldots \\
0 & 0 & -\frac{\cos \Phi_{3}}{l_{3}} & -\frac{\sin \Phi_{3}}{l_{3}} & \ldots \\
0 & 0 & \sin \Phi_{3} & -\cos \Phi_{3} & \ldots \\
\frac{\sin \Phi_{1}}{l_{1}} & -\frac{\cos \Phi_{1}}{l_{1}} & -\frac{\sin \Phi_{1}}{l_{1}} & \frac{\cos \Phi_{1}}{l_{1}} & \ldots \\
0 & 0 & \frac{\sin \Phi_{2}}{l_{2}} & -\frac{\cos \Phi_{2}}{l_{2}} & \ldots \\
0 & 0 & 0 & 0 & \ldots \\
0 & 0 & 0 & 0 & \ldots \\
0 & 0 & \cos ^{2} \Phi_{3} & \cos \Phi_{3} \sin \Phi_{3} & \ldots \\
0 & 0 & \cos \Phi_{3} \sin \Phi_{3} & \sin ^{2} \Phi_{3} & \ldots
\end{array}\right.
$$

$\begin{array}{lccccc}\ldots & 0 & 0 & 0 & 0 & \ldots \\ \ldots & \cos \Phi_{2} & \sin \Phi_{2} & 0 & 0 & \ldots \\ \ldots & 0 & 0 & -\cos \Phi_{3} & -\sin \Phi_{3} & \ldots \\ \ldots & 0 & 0 & 0 & 0 & \ldots \\ \ldots & 0 & 0 & \frac{l_{3} t \cos \Phi_{3}+s \sin \Phi_{3}}{l_{3}^{2}} & \frac{l_{3} t \sin \Phi_{3}-s \cos \Phi_{3}}{l_{3}^{2}} & \ldots \\ \ldots & 0 & 0 & -t \sin \Phi_{3} & t \cos \Phi_{3} & \ldots \\ \ldots & 0 & 0 & 0 & 0 & \ldots \\ \ldots & -\frac{\sin \Phi_{2}}{l_{2}} & \frac{\cos \Phi_{2}}{l_{2}} & 0 & 0 & \ldots \\ \ldots & 0 & 0 & \frac{\sin \Phi_{3}}{l_{3}} & -\frac{\cos \Phi_{3}}{l_{3}} & \ldots \\ \ldots & 0 & 0 & 0 & 0 & \ldots \\ \ldots & 0 & 0 & \frac{\sin \Phi_{3}\left(-s \cos \Phi_{3}+t l_{3} \sin \Phi_{3}\right)}{l_{3}} & \frac{\cos \Phi_{3}\left(s \cos \Phi_{3}-t l_{3} \sin \Phi_{3}\right)}{l_{3}} & \ldots \\ \ldots & 0 & 0 & -\frac{\sin \Phi_{3}\left(l_{3} t \cos \Phi_{3}+s \sin \Phi_{3}\right)}{l_{3}} & \frac{\cos \Phi_{3}\left(t l_{3} \cos \Phi_{3}+s \sin \Phi_{3}\right)}{l_{3}} & \ldots\end{array}$

$$
\begin{array}{lcccc}
\ldots & 0 & 0 & 0 & 0 \\
\ldots & 0 & 0 & 0 & 0 \\
\ldots & \cos \Phi_{3} & \sin \Phi_{3} & 0 & 0 \\
\ldots & -\cos \Phi_{4} & -\sin \Phi_{4} & \cos \Phi_{4} & \sin \Phi_{4} \\
\ldots & \frac{l_{3}(t-1) \cos \Phi_{3}-s \sin \Phi_{3}}{l_{3}^{2}} & \frac{l_{3}(t-1) \sin \Phi_{3}+s \cos \Phi_{3}}{l_{3}^{2}} & 0 & 0 \\
\ldots & -(t-1) \sin \Phi_{3} & (t-1) \cos \Phi_{3} & 0 & 0 \\
\ldots & 0 & 0 & 0 & 0 \\
\ldots & 0 & 0 & 0 & 0 \\
\ldots & -\frac{\sin \Phi_{3}}{l_{3}} & \frac{\cos \Phi_{3}}{l_{3}} & 0 & 0 \\
\ldots & \frac{-\frac{\cos \Phi_{4}}{l_{4}}}{l_{4}} & -\frac{\sin \Phi_{4}}{l_{4}} & \frac{\cos \Phi_{4}}{l_{4}} \\
\ldots & \frac{\sin \Phi_{3}\left(s \cos \Phi_{3}+l_{3} \sin \Phi_{3}-t l_{3} \sin \Phi_{3}\right)}{l_{3}} & \frac{\cos \Phi_{3}\left(-s \cos \Phi_{3}+l 3(t-1) \sin \Phi_{3}\right)}{l_{3}} & 0 & 0 \\
\ldots & \frac{\sin \Phi_{3}\left(l_{3}(t-1) \cos \Phi_{3}+s \sin \Phi_{3}\right)}{l_{3}} & -\frac{\cos \Phi_{3}\left(l_{3}(t-1) \cos \Phi_{3}+s \sin \Phi_{3}\right)}{l_{3}} & 0 & 0 \\
& & & (4.0)
\end{array}
$$


In analogy to equations (4.34) and (4.35), we calculate the energy gradients with the common prefactors defined as:

$$
\begin{array}{ll}
A:=-\frac{\partial E_{\mathrm{sb}}(\tilde{\alpha}, s)}{\partial \tilde{\alpha}}=-\left(1-\frac{s}{s^{*}}\right) \frac{\left(c+c^{2}\right)^{\eta / 2} \eta \cos \tilde{\alpha} \sin \tilde{\alpha}\left(\frac{1}{c}-\frac{1}{c+\sin ^{2} \tilde{\alpha}}\right)^{\eta / 2-1}}{\left(c+\sin ^{2} \tilde{\alpha}\right)^{2}} \\
B:=-\frac{\partial E((\tilde{\alpha}, s)}{\partial s}= & \beta\left(c+c^{2}\right)^{\eta / 2}\left(\frac{1}{c}-\frac{1}{c+\sin ^{2} \alpha}\right)^{\eta / 2} \frac{1}{s^{*}}
\end{array}
$$

The forces then become:

$$
\begin{aligned}
\mathbf{F}_{1}= & A \frac{1}{2 l_{1}}\left(\begin{array}{c}
-\sin \Phi_{1} \\
\cos \Phi_{1}
\end{array}\right) \\
\mathbf{F}_{2}= & A\left[\frac{\Phi_{3}-\Phi_{4}}{l_{3}}\left(\begin{array}{c}
-\cos \Phi_{3} \\
-\sin \Phi_{3}
\end{array}\right)+\frac{1}{2 l_{2}}\left(\begin{array}{c}
\sin \Phi_{1} \\
-\cos \Phi_{1}
\end{array}\right)+\frac{1}{2 l_{2}}\left(\begin{array}{c}
-\sin \Phi_{2} \\
\cos \Phi_{2}
\end{array}\right)\right]-B \hat{s} \\
\mathbf{F}_{3}= & A \frac{1}{l_{2}}\left(\begin{array}{c}
\sin \Phi_{2} \\
-\cos \Phi_{2}
\end{array}\right) \\
\mathbf{F}_{4}= & A\left[\frac{\Phi_{3}-\Phi_{4}}{l_{3}} t\left(\begin{array}{c}
\cos \Phi_{3} \\
\sin \Phi_{3}
\end{array}\right)+\frac{\Phi_{3}-\Phi_{4}}{l_{3}} \hat{s}+\frac{t+\frac{1}{2}}{l_{3}}\left(\begin{array}{c}
\sin \Phi_{3} \\
-\cos \Phi_{3}
\end{array}\right)\right]+B t \hat{s} \\
\mathbf{F}_{5}= & A\left[\frac{\Phi_{3}-\Phi_{4}}{l_{3}}(t-1)\left(\begin{array}{c}
-\cos \Phi_{3} \\
-\sin \Phi_{3}
\end{array}\right)-\frac{\Phi_{3}-\Phi_{4}}{l_{3}} \hat{s}+\frac{t+\frac{1}{2}}{l_{3}}\left(\begin{array}{c}
-\sin \Phi_{3} \\
\cos \Phi_{3}
\end{array}\right)\right. \\
& \left.+\left(t-\frac{1}{2}\right)\left(\begin{array}{c}
-\sin \Phi_{4} \\
\cos \Phi_{4}
\end{array}\right)\right]-B(t-1) \hat{s} \\
\mathbf{F}_{6}= & A \frac{\left(t-\frac{1}{2}\right)}{l_{4}}\left(\begin{array}{c}
\sin \Phi_{4} \\
-\cos \Phi_{4}
\end{array}\right)
\end{aligned}
$$

Here $\hat{s}$ is the unit vector pointing from $\mathbf{P}$ to $\mathbf{r}_{\mathbf{2}}$.

In the following, we will again break these terms down into the different physical interactions taking place in this scenario.

\section{Aligning Torques}

The torque forces from equation (4.35) reappear, with the exception that $t$ is here fixed to 0 in the case of the first fiber, since the stretched bridge is tethered to $\mathcal{N}_{2}$. 


\section{Sliding Forces and Torques}

The first terms in $\mathbf{F}_{4}$ and $\mathbf{F}_{\mathbf{5}}$ are the familiar shift forces inducing a parallel shift of the fibers along each other, moving the point of attack $\mathbf{P}$ to areas of smaller interpolated contact angle. It is compensated by an equal and opposite counter-force acting on $\mathcal{N}_{2}$.

The new terms appearing in $\mathbf{F}_{4}$ and $\mathbf{F}_{5}$ correspond to rotational mode of segment $\mathcal{S}_{3}$ that would also lead to a shift of point $\mathbf{P}$ because of the orthogonality condition for the stretched bridge.

\section{Attractive Forces}

Lastly, the terms after the prefactors $B$ are the constant central attraction forces of the stretched bridge. This acts directly on node $\mathcal{N}_{2}$, and is distributed among nodes $\mathcal{N}_{4}$ and $\mathcal{N}_{5}$ according to the respective lever lengths.

\section{Modifications in the Case of End Segments}

As in the case of sliding contact bridges, in the case of segment $\mathcal{S}_{3}$ not having a nearest neighbor, it is assumed to be straight, and hence the parallel shift forces disappear. The same holds for the additional torque term, which arose from the orthogonal construction of the stretched bridge. This implies that the same terms are still valid for the case of point $\mathbf{P}$ coinciding with the node $\mathcal{N}_{4}$, in which case the orthogonality assumption doesn't hold anymore.

In the case of either $\mathcal{S}_{1}$ or $\mathcal{S}_{2}$ not existing, prefactors of $1 / 2$ in the terms of $\mathbf{F}_{1} \ldots \mathbf{F}_{3}$ change to 1 , again in analogy to the contact bridge case.

As before, explicit calculation of the three-segment scenario confirms these modifications.

\subsection{Numerical Integration of Equations of Motion}

To simulate the dynamics of the system, Newton's equations of motion are integrated. In the following, we will use typewriter font to indicate that we are talking about the computer representation of an object, such as a segment or a bridge.

In order to analyze equilibrium configurations or quasistatic deformations, it is usually preferable to use an energy minimization technique such as the conjugate gradient method. However, these methods are only effective because they are allowed to use large displacements in order to find the minimum 
energy configuration. Since we employ bridge objects that can be created or destroyed according to dynamical events such as fibers coming into contact or losing contact, we need to take care that these events follow from the physical dynamics of the system. To this end, we use Newton's equations of motion, together with a damping term to ensure that the dynamics are overdamped.

Crosslinks between fibers are implemented by an additional object type, called a supernode. This is placed at the position of the crossing point, and the nodes of the two crossing fibers at that point are associated with it. The liquid bridges are called bridges when the fibers are in contact. Stretched bridges have a slightly different structure, since they are associated with up to two segments and one node. They are called sbridges.

The forces arise from the deformation of segments (stretching), nodes (bending) and ( $\mathrm{s}$ ) bridges (capillary action), as well as a damping term of a viscous background fluid. This last term manifests in a force on the nodes that is proportional to their velocity. All force terms are accumulated in the supernodes and divided by the sum of their subnode's effective lengths (or dimensionless masses) to give accelerations. At each time step, the set of bridges has to be reevaluated:

- The cross finding algorithm identifies new crossing points and generates bridges

- bridges' associated segments are updated as they slide along the fibers

- Loss of fiber contact is detected, and bridges are transformed into sbridges accordingly

- When fibers reconnect, sbridges are converted back.

- sbridges are deleted when stretched beyond the rupture distance $s^{*}$.

Only after these updates can the new forces be calculated for the next time step.

The following forces act on any given supernode:

- The stretching forces 4.21 of each connected segment

- The bending force 4.17) of the nodes themselves if they have neighbors, and either 4.18) or 4.19) if a neighboring node is bent

- The corresponding force terms from equations 4.35) or 4.42 if the node is part of the minimal subsystem of a bridge or stretched bridge 
- An inertial force $\mathbf{F}_{a}=\tilde{l}_{i}^{0} \mathbf{a}_{i}$ proportional to the acceleration $\mathbf{a}_{i}$ of the supernode

- A damping force $\mathbf{F}_{d}=-\gamma \mathbf{v}_{i}$ proportional to the supernode's velocity $\mathbf{v}_{i}$ and the damping coefficient $\gamma$.

The damping coefficient $\gamma$ is chosen such that the dynamics of the system are overdamped.

\section{Verlet Algorithm}

After the network is initialized, periodic or Lees-Edwards boundary conditions are enforced, and then the following steps are taken repeatedly until the kinetic energy of the system converges.

This implements the Velocity Verlet integration scheme AT89, which is frequently used in molecular dynamics simulations because it offers great stability at a very low computational cost.

1. If a wet network is being simulated, the set of $(\mathrm{s})$ bridges is updated by recalculating the crossing points of fibers and detecting contact and rupture events.

2. The sum of all forces on the supernodes are recalculated by setting them to zero and then looping over all segments, nodes and $(s)$ bridges to calculate the forces according to equations (4.21), 4.17)-4.19), 4.35) and (4.42) and added to the respective nodes. A frictional force term $-\gamma \mathbf{v}$ is added at the end.

3. The first half-step of the velocity-Verlet algorithm: The accelerations of all supernodes are set to $\mathbf{F} / \tilde{l}^{0}$, supernodes are displaced by $\Delta \mathbf{x}=$ $\dot{\mathbf{x}} \Delta t+\frac{1}{2} \ddot{\mathbf{x}} \Delta t^{2}$. Velocities are adjusted by $\Delta \mathbf{v}=\frac{1}{2} \ddot{\mathbf{x}} \Delta t$.

4. Positions are made to conform to the periodic or Lees-Edwards boundary conditions again for detection of crossings. Forces are recalculated as above.

5. The second half-step: Accelerations are set to $\mathbf{F} / \tilde{l}^{0}$ again; velocities are increased by $\Delta \mathbf{v}=\frac{1}{2} \ddot{\mathbf{x}} \Delta t$.

During these simulations, elastic, kinetic and interfacial energies are continuously measured, along with the numbers of bridges and the components of 
the elastic and capillary stress tensors. The latter is calculated by evaluating the force components of all forces transferred across a vertical or horizontal cut through the system, and averaged over a number of such cuts.

\section{Step size control}

The energy landscape of wet network systems as described in this chapter can occasionally become very steep. When it does, numerical accuracy can only be maintained by reducing the time step of the integrator. In the following, the problematic configurations will be outlined and the strategy used to adapt the simulation time step is presented.

When the two segments cross at a very small angle, some of the gradient terms involving $1 / \sin \alpha$ in 4.35 become very large. This is not a problem as long as the interpolated angle $\tilde{\alpha}$ is also small, since the prefactor $A$ also vanishes in that case. If however a neighboring segment bends away strongly and the crossing segments are perfectly aligned, the curvature force pushing the crossing point to the middle of the segment becomes very large. An adaptive step size algorithm must ensure that the motion arising from this force can be resolved to the local minimum of this steep potential well (the point where both segments cross in the middle).

Let the large force term arising from this situation be $\mathbf{F}$, and the corresponding generalized variable of segment shift $t$. Then a sufficiently small time step is

$$
\tau=\sqrt{\frac{l_{\text {eff } l \sin \alpha}}{t|\mathbf{F}|}} .
$$

When a bridge exerts a force larger than a certain threshold value, the corresponding $\tau$ is calculated, the first half-step of the Verlet algorithm is rolled back, and the step is repeated with a time step of $0.1 \tau$ until the suggested minimum time step does not decrease below $90 \%$ of the currently used one anymore. Only then is the next step again taken with the normal time step.

\subsection{Capillary Adhesion of Fibers}

The model as presented so far in this chapter is a way of describing and simulating elastic fibers with an adhesive interaction. In it, the interaction is 


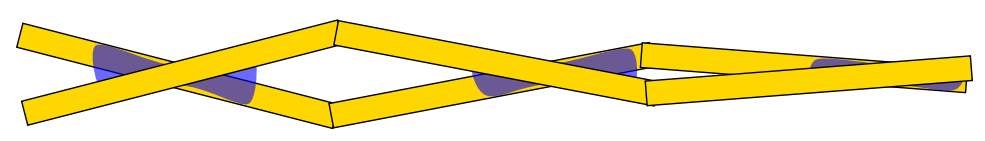

Figure 4.13: Illustration of a fiber bundle adhering by liquid bridges, with exaggeratedly large crossing angles.

closely modeled after capillary interactions, which have some special properties, such as aligning torques arising from the liquid morphologies, and a lack of resistance to parallel translations. Nevertheless, primarily, the interaction manifests in adhesion of fibers. There is a certain adhesion energy associated with liquid bridges, and a wet fiber system in mechanical equilibrium will be characterized by a balance between adhesion forces and elastic forces: The adhesion forces will cause fibers to align into parallel overlapping bundles, thus gaining adhesion energy, but the necessary deformations are associated with a cost in elastic energy.

This interpretation of the model potential (4.36) as an adhesion energy comes from the fact that the interaction leads to alignment of crossing fibers. Therefore, the energetically preferred state is that where the full length of the segment is parallel (and therefore in contact to) the crossing segment. In a bundle of fibers that have completely aligned, the associated energy per segment length is the energy difference between that of two segments crossing at right angles and that of two aligned segments. In the system of units presented in section 4.1.2, this is just $\beta$. This is the adhesion energy per segment length. Each pair of crossing segments in a bundle contributes one liquid bridge, so that $\beta$ also becomes the adhesion energy per average segment length in a bundle of two fibers adhering by capillary action.

In the simulations, such a bundle is formed by chains of segments that are almost completely aligned, but keep crossing each other (see figure 4.13). The rules implemented in the simulation for tracking stretched bridges ensure that the number of bridges always corresponds to the number of paired segments: A stretched bridge generated by a kink slipping off (and therefore by two bridges disappearing) covers all four involved segments and accounts for the forces and energies of the destroyed bridges. Segment pairs that are covered by stretched bridges cannot form new contact bridges. All this ensures that the total energy of all bridge objects in a fiber bundle is consistent with the picture of a constant adhesion energy per length. 


\subsubsection{Analytical Solutions for Fibers Rings Adhering to a Wall}

The problem of calculating equilibrium shapes of elastic rods or fibers, i.e., the study of the variational problem of minimizing Kirchhoff's elastic energy (4.3) under certain boundary conditions goes back to Euler, who neglected the extensional strain part. This reduced problem is known as the Elastica problem, see for example chapter XIX of [Lov44].

The problem of a two-dimensional representation of lipid vesicles attached to a wall has been studied by Seifert in [SL90]. This problem is another example of competition between adhesion and elastic deformation; furthermore, the elastic energy of a two-dimensional cut through a vesicle wall is also described by a Hamiltonian that is quadratic in the curvature, which is in this case usually referred to as the Helfrich Hamiltonian [DH76]. Seifert considers either the case of a constant area enclosed by the vesicle wall, or a constant pressure difference between the inside and the outside of the vesicle. Replacing the vesicle wall with a ring-shaped fiber, only the case of equal pressures makes physical sense, since there is no energy penalty for changing the enclosed area.

We use this scenario in order to compare the results of simulations of wet fiber rings adhering to a wall to analytical calculations.

In order to calculate equilibrium shapes of a fiber adhering to a substrate by finding the centerline curves that minimize the total energy, one needs to minimize the sum of the elastic energy and an adhesion energy. For a fiber adhering to a wall over the arc-length range $s \in\left(s_{c}, L\right)$, where $s_{c}$ defines the point of contact, where the fiber first adheres, we can write:

$$
E_{\mathrm{ad}}=\beta\left(L-s_{c}\right)
$$

for the adhesion energy. Here, $\beta$ is again the adhesion energy per unit length measured in terms of the bending stiffness $\kappa$.

From the extensive literature concerned with the Euler Elastica, it is known that for the variational problem, it is easiest to parametrize the curve described by the fiber by giving the orientation $\Phi$ as a function of arc length $s$ (see figure 4.14). Exploiting the mirror symmetry of the problem, we define the origin of $s$ to be at the top and middle of the adhered fiber ring, as shown in the figure. In this parametrization, the curvature is simply given by the derivative of the orientational angle with respect to the arc length: $C=\dot{\Phi}$. 


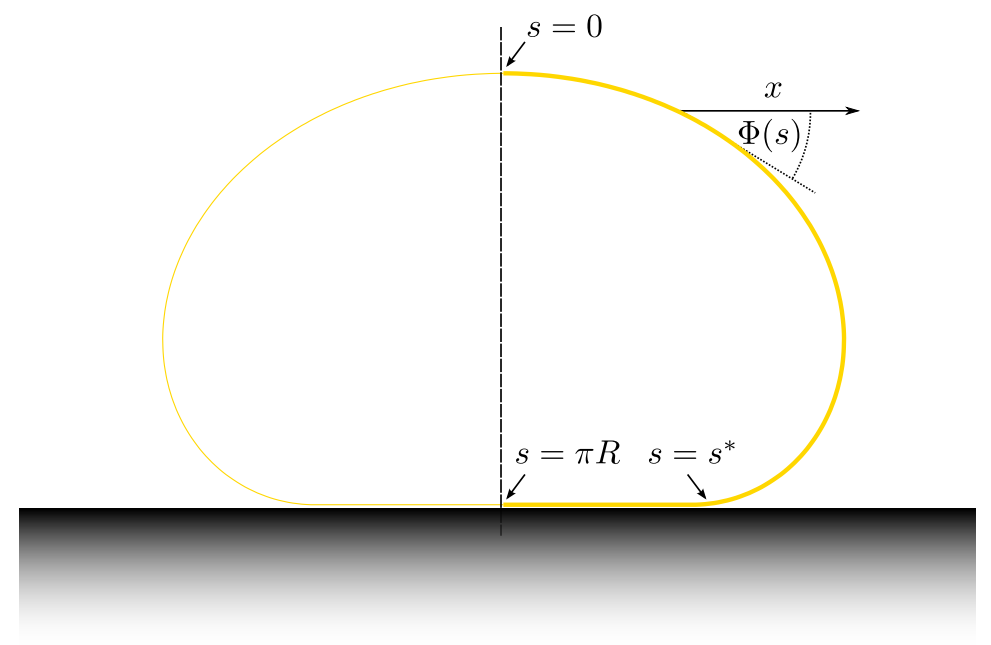

Figure 4.14: A fiber ring adhering to a wall. The shape is described by a function $\Phi(s)$.

The Cartesian positions of a point on the fiber at arc length $s$ are then:

$$
\begin{aligned}
& x=\int_{0}^{s} \mathrm{~d} s \cos \Phi(s) \\
& y=-\int_{0}^{s} \mathrm{~d} s \sin \Phi(s) .
\end{aligned}
$$

The elastic energy is then written as

$$
\int_{0}^{s_{c}} \mathrm{~d} s\left(\frac{1}{2} \dot{\Phi}^{2}+\gamma(\dot{x}-\cos \Phi)\right) .
$$

Here, $\gamma(s)$ is a Lagrange multiplier used later on to enforce a certain lateral position of the end of the fiber.

The variational problem of finding the shape $\Phi(s)$ of a fiber that minimizes (4.48) is equivalent to the Euler Elastica problem, and it is also equivalent to the Euler-Lagrange problem of the gravitational pendulum, when one interprets $s$ as the time and $\Phi$ as the phase of the pendulum. This is known as the Kirchhoff analogy, and was used by Kirchhoff in his study of flexible rods Kir59 that was mentioned earlier in section 4.1 


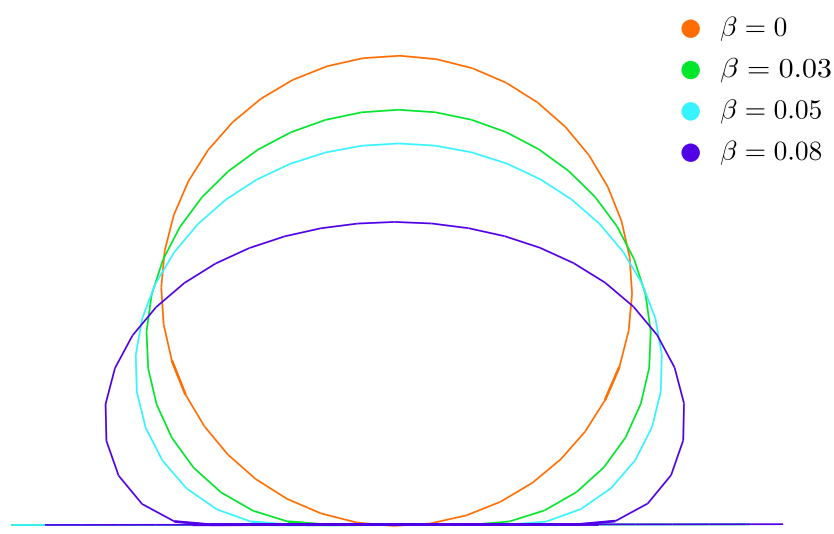

Figure 4.15: Shapes of a fiber ring with a circumference of 20 segments that is attached to a wall (simulated by a stiff fiber) at different capillary strengths $\beta$.

The solution can be expressed in closed form using Jacobi elliptic functions:

$$
\Phi(s)= \pm 2 \mathrm{am}\left(s \sqrt{\frac{A-\gamma}{2}},-\frac{2 \gamma}{A-\gamma}\right),
$$

Here, $A$ is an integration constant and $\operatorname{am}(\Phi, k)$ is the Jacobi amplitude, defined as the inverse $\Phi$ of the elliptic integral of the first kind (see [BSMM01]):

$$
u=\int_{0}^{\Phi} \frac{\mathrm{d} \psi}{\sqrt{1-k^{2} \sin ^{2} \psi}} ; \quad \operatorname{am}(u, k)=\Phi .
$$

To apply this to a ring-shaped fiber adhering to a wall as depicted in figure 4.14, one needs a boundary condition at the point $s=s_{c}$ where adhesion starts. This can be derived by variation of the contact point $s_{c}$. Moving the contact point by a small amount $\delta s$ so that a small part of adhering fiber is loosened, work of the order $\beta \delta s$ has to be done against the adhesion potential. On the other hand, since the part that formerly adhered to a straight wall with zero curvature is now going to be curved, the bending moment of the fiber will do an amount of work equal to $1 / 2 \dot{\Phi}\left(s_{c}\right)^{2} \delta s$. In equilibrium, these terms will be equal, so that we get:

$$
\dot{\Phi}\left(s_{c}\right)=\sqrt{2 \beta}
$$


as a boundary condition for the curvature of an adhering fiber. In the appendix of [SL90], Seifert gives a more general derivation of this boundary condition.

The orientation $\Phi$ itself must be continuous in $s$, since discontinuities correspond to infinite curvatures and thus infinite elastic energies. Therefore, another boundary condition is that $\Phi\left(s_{c}\right)=\pi$ (see figure 4.14). Using these conditions and the solution (4.49), the integration constant $A$ and the Lagrange multiplier $\gamma$ can be expressed in terms of the adhesion energy per unit length $\beta$ and the curvature $C_{0}=\dot{\Phi}(0)$ at $s=0$ :

$$
\begin{aligned}
& A=\frac{1}{4}\left(C_{0}^{2}+2 \beta\right) \\
& \gamma=\frac{1}{4}\left(2 \beta-C_{0}^{2}\right) .
\end{aligned}
$$

The curvature $C_{0}$ for a given adhesion potential $\beta$ is uniquely determined, but can only be calculated numerically, since it is the solution of an equation involving elliptic functions. The solid line in figure 4.17 shows the length of the adhered part as a function of $\beta$ for a circle of radius $R=40$. Below $\beta=$ $1 /\left(2 R^{2}\right)$, there is no solution: Circles of unit radius do not adhere to straight walls when the dimensionless adhesion energy per unit length is smaller than $1 / 2$.

Figure 4.15 shows simulations of ring-shaped fibers adhering to a straight wall by liquid bridges for several capillary interaction strengths $\beta$, simulated using our model for wet fiber networks. In our model, $\beta$ is effectively equivalent to the dimensionless adhesion energy per segment length, as explained in the beginning of this section. To verify this, we fit the analytical shapes calculated by (4.49) to the positions of the nodes in the simulations for various $\beta$, using $C_{0}$ as a fit parameter. Figure 4.16 shows such a fit. From the fit parameter $C_{0}$, one can calculate the adhesion potential $\beta$ that corresponds to the analytically calculated shape that fits the data best.

It is to be noted that the equilibrium state reached in a simulation will always differ from the prediction in the continuum model, since the liquid bridge objects discretize the adhered part. In order to generate one more bridge, the first unbound node has to be bent until the first unbound segment touches the wall. Therefore, there is an energy barrier between equilibrium states, and an associated hysteresis.

This effect can be seen in figure 4.17. Here, we plotted the length $L^{*}$ of the adhered part of the fiber as a function of $\beta$ for a circular fiber of circumference $40 l_{s}$ as an orange line. The blue circles correspond to measurements taken 


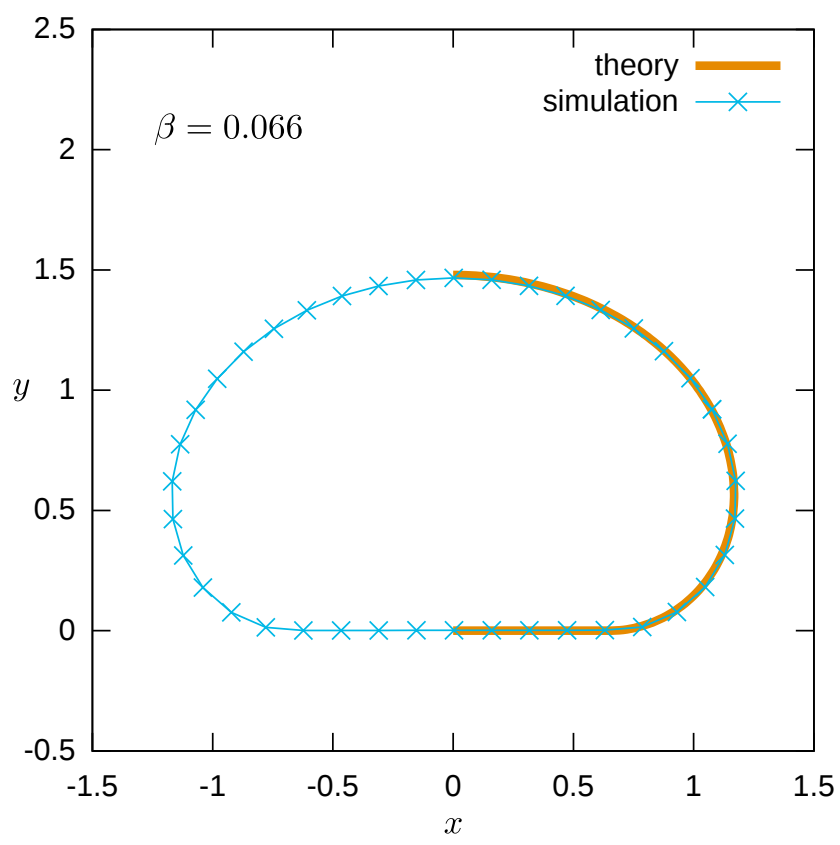

Figure 4.16: Comparison of the analytically calculated shape of an adhered fiber ring (shown only in the right half) with a simulation of a wet fiber ring sticking to a wall by capillary adhesion. The chosen parameters are $\beta=0.066$ for the simulation and $\beta=0.05$ for the analytical shape. The simulated ring has a circumference of 40 segments and hence a radius of $R \approx 6.4$. Apart from a random horizontal shift of the simulated ring, $C_{0}$ is the only fit parameter

from simulations of a circle touching the wall in one point initially. Adhesion sets in at the point predicted from the continuum model, but the adhered length stays below the predicted value after that.

The blue triangles are taken from simulations that used the final state of the simulation at $\beta=0.08$ as a starting condition. Here, the adhered length measured stays above the predicted value. This is an effect of the hysteresis caused by the discretization. Nevertheless, the critical value $\beta_{c}$ could be reproduced, and the values of $L^{*}$ qualitatively follow the behavior predicted by the continuum model.

These measurements confirm that simulations carried out in the way described in this chapter, with liquid bridge objects being created at segment crossing points, are consistent with the picture of elastic fibers that interact with each other via an adhesion per unit length of magnitude $\beta$. This adhe- 


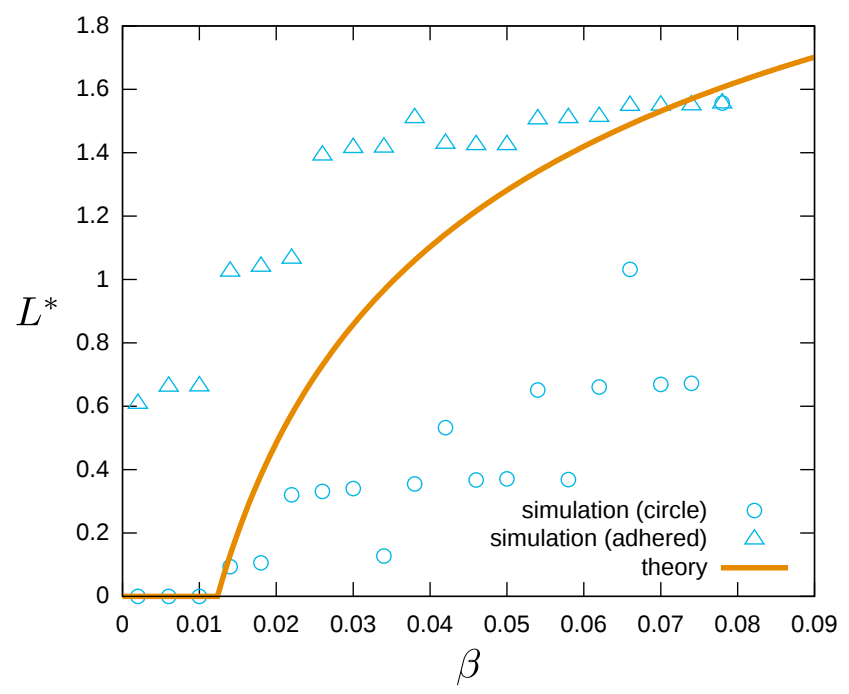

Figure 4.17: Adhered length of a circular fiber as a function of the adhesion energy/length. The circles represent simulated systems where the initial condition was an undistorted circular fiber touching a wall, while the triangles represent simulations where the initial condition was an already adhered fiber (taken as the final configuration of the simulation at $\beta=0.08$.)

sion potential has a finite range, which is equal to zero for fibers that don't already interact, and equal to the rupture distance $s^{*}$ for interacting fibers, i.e., it is in itself hysteretic.

Additionally, hysteresis is induced by the discretization itself. Locally, the interaction implemented here involves torques that are consistent with those induced by capillary action between cylinders.

\subsection{Network Deformation by Weak Liquid Bridges}

In this section, we will present our analysis of the morphological and mechanical properties of wet Mikado networks. These have been simulated according to the procedure detailed above in order to find the equilibrium configurations. In the course of such a simulation, the internal stresses caused by the liquid bridges act to deform the elastic network, allowing more bridges to be created while the fibers align (corresponding to continued capillary condensation while the action of the liquid interface brings fibers closer together). 


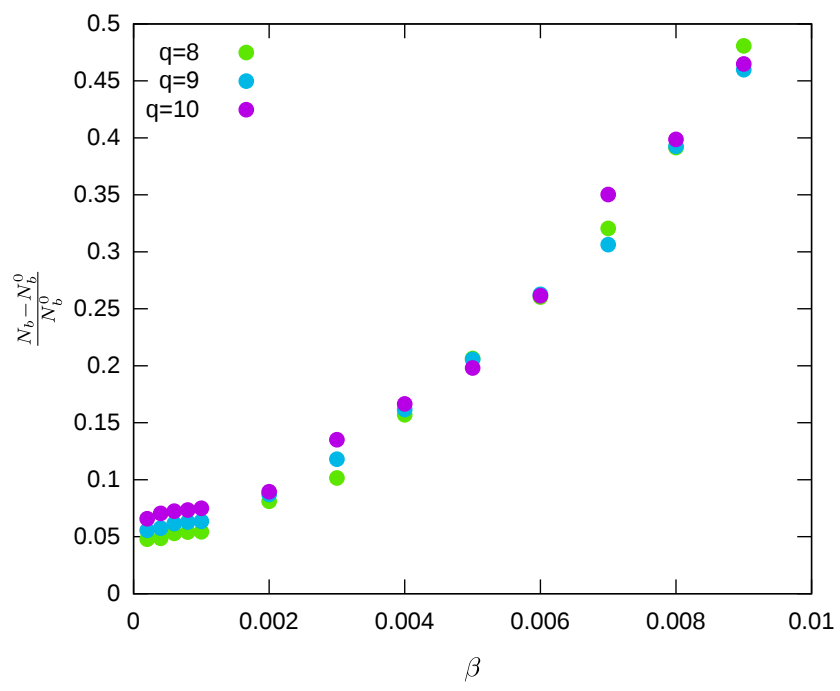

Figure 4.18: The relative increase in the number of liquid bridges on increasing the capillary strength $\beta$, plotted for various network densities $q$.

The space of control parameters we chiefly explore is spanned by the fiber density $q$ of the network and the capillary interaction parameter $\beta$, governing the magnitude of the force a liquid bridge can exert in terms of the elastic modulus of the fibers. We use an exponent of $\eta=-1$ for the angular dependence $E \propto \alpha^{-\eta}$ of the liquid bridges (see section 4.2.1), corresponding to the constant pressure ensemble, where the bridges exchange volume with a vapor phase permeating the system. The aspect ratio of the unit segments is fixed to $a=0.5$, meaning that the length and radius of a fiber have a ratio of 1:20. This leads to elastic fibers that primarily deform by bending. The system sizes we were able to simulate in the given parameter ranges are $L=12 l_{f}$.

First, we study the increase of the number of bridges in the system due to the action of the bridges at low values of $\beta$. Figure 4.18 shows the relative increase in the number of liquid bridges on increasing $\beta$, for a small selection of different network densities $q$. The crosslinking of the networks presented here was performed with a probability of $p_{l}=0.5$.

There is a steady increase in the number of bridges of the order of $5 \%$ even for very low values of $\beta$; this is caused by the liquid bridges present from the start that act on fibers that do not need to deform much in order to form new contacts. This initial increase shows a slight dependency on the fiber density $q$. Networks of higher density have fewer easily deformable fibers (since they are 
further along the percolation transition). They also have more liquid bridges initially, and the fibers are closer together, meaning that less deformation is necessary to form new contacts. The latter effect seems to dominate, since denser networks have a slightly larger initial increase of bridges.

For $\beta \lesssim 10^{-3}$, an increase of the capillary strength has no noticeable effect on the liquid content. Only when $\beta$ reaches values around $\beta \approx 5 \cdot 10^{-3}$ does the effect of $\beta$ on the liquid content become significant. This is the regime where formation of new liquid bridges creates enough additional stress in the network that the additional aligning forces keep the process going, and more and more fiber segments are drawn into the developing fiber bundles. This collective effect leads to the segmentation of the network into bundles and pores, as we will describe later on in section 4.6 .

Only when $\beta$ becomes of order $10^{-2}$ could we observe significant morphological changes in the network. In the regime of lower $\beta$, we resort to the statistics of the fiber crossing angles $\alpha$ to give information about the deformations that the weak liquid bridges induce.

Figure 4.19 shows how the distribution of crossing angles $\alpha$ varies on increasing the capillary strength $\beta$ in the regime where no collective increase of crossings occurs. The distribution roughly follows the undeformed sine-like distribution (3.1) derived in chapter 3 . We use this to characterize the deformations induced by the wetting liquid in the regime of low $\beta$, where large scale rearrangements of the network do not yet take place. The blue lines in figure 4.19 mark the measured frequencies of a given angle $\alpha$, with the darkness of the line increasing with the capillary strength $\beta$. The gray line is taken from equation (3.1). While the differences in the distributions are small, it is still evident that the crossing points in the lowest $10 \%$ of crossing angles are significantly increased with increasing $\beta$, while crossings closer to $\alpha=\pi / 2$ tend to be depleted. In the broad middle range of the spectrum of crossing angles, no significant change can be observed.

Looking at figure 4.4(b), it is evident that the largest torques act on the points where the crossing angles are already low, so that the trend in the plot 4.19 is to be expected. Other than the alignment of fibers that already cross at relatively low angles (and corresponding very low increase in the number of liquid bridges as seen in figure 4.18), no significant effect of the wetting liquid on the network structure could be detected in this regime of weak capillary interactions. 


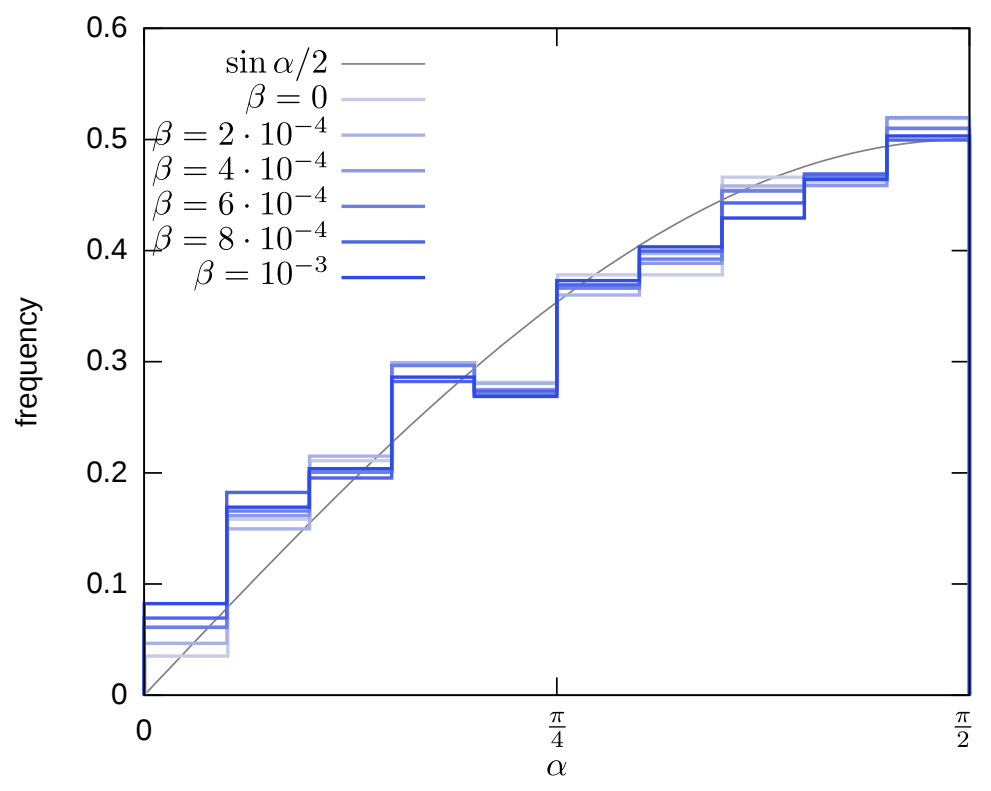

Figure 4.19: Distribution of crossing angles $\alpha$ in a network at density $q=10$, on increasing the capillary strength $\beta$.

\subsection{Observations about Networks Deformed by Strong Liquid Bridges}

Increasing $\beta$ to larger values, we observe the formation of bundles and pores in the network. This is a cooperative effect, caused by the capillary action leading to alignment and therefore to formation of new liquid bridges, until large accumulations of bridges along bundles of fibers occur. Figure 4.20(a) shows an example of such a network. The fibers are plotted in yellow, while the liquid bridges are shaded in blue. Fluctuations in the density of fibers and crosslinks lead to a coarsening effect, where liquid accumulates in small regions of perfectly aligned fibers, depleting the surrounding areas of fiber material. Other areas, where the network is more densely linked, only deform slightly, and the number of liquid bridges in those areas remains relatively small.

This leads to the formation of pores bounded by bundles of fibers. Figure 4.20(b) shows the distribution of elastic stress in the network. Fibers drawn with a large line thickness in this image are more stressed than the thinner fibers. The color scale denotes the ratio of the bending energy of the fiber segment to its total elastic energy, meaning that fibers plotted in red are deformed predominantly by bending, while blue fibers are mainly stretched. 


\subsection{Observations about Networks Deformed by Strong Liquid Bridges}

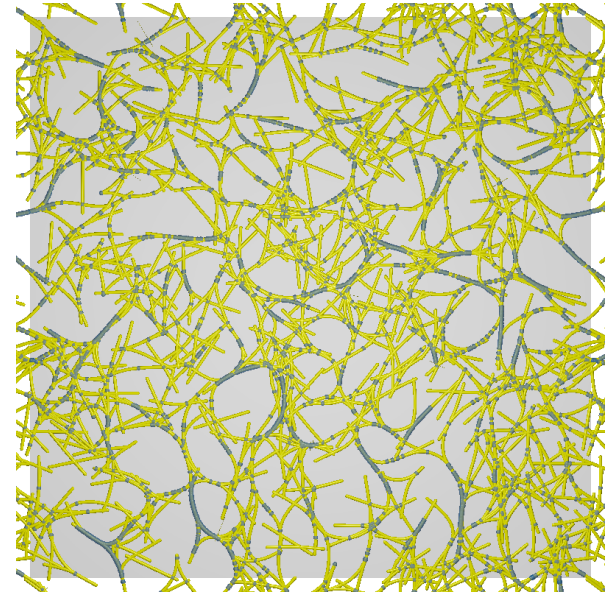

(a)

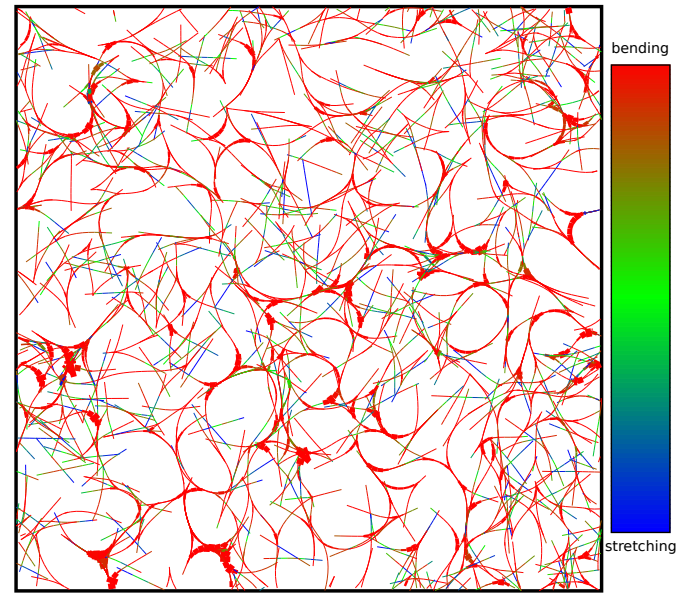

(b)

Figure 4.20: A wet elastic network of $10^{3}$ fibers with a fiber density of $q=10.00$ and an elastocapillary parameter of $\beta=0.04$ in its ground state.(a) Distribution of the 5400 capillary bridges that have formed (light blue). The shaded area depicts the simulation box, which has a size of $10 l_{\text {fib }}$ in this case. (b) Distribution of elastic energies. Line thickness denotes total elastic energy of a segment (with a minimum thickness so that all lines are visible), while the color depicts how the elastic energy is distributed between stretching and bending modes.

It is obvious that the elastic stress builds up at places where the fibers have to bend at the ends of the bundles adhering by liquid bridges. These bundles are bordered by dense regions that have not deformed significantly, and in the boundary regions, bending takes place in order to accommodate the alignment into bundles. Within the bundles themselves, stresses are not very high, meaning that the fibers were absorbed into the bundles exciting only relatively soft modes of deformation.

Figure 4.21 is shows the initial and relaxed states of a network with the structure of the rigid clusters superimposed. The networks were segmented into rigidly connected clusters with the pebble game algorithm as described in section 3.4. There is a percolating rigid cluster colored in purple, along with some smaller rigid clusters in other colors and some floppily connected segments drawn in gray. 


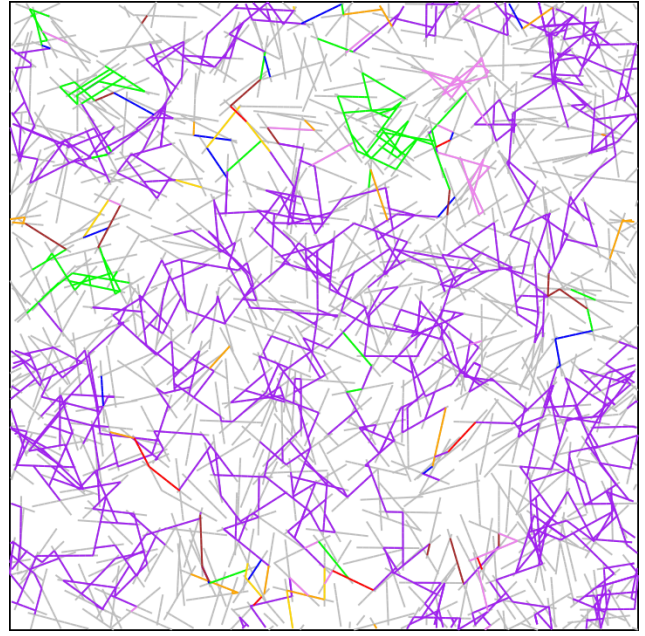

(a)

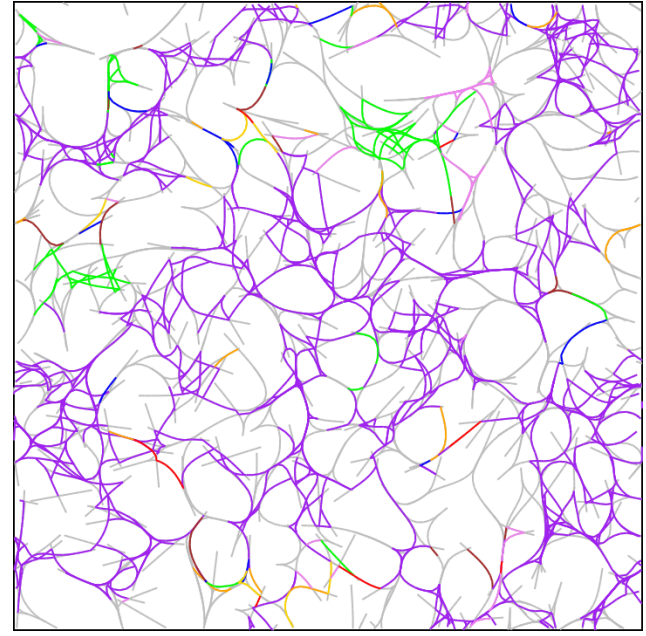

(b)

Figure 4.21: A network at density $q=10$, with the rigid clusters colored. Grey fibers are not part of a rigid cluster. Figure 4.21(a) shows the initial, undeformed state, while figure 4.21(b) shows the network after relaxation with a capillary strength of $\beta=0.1$.

Comparing the morphology of the relaxed network to the structure of rigid clusters, it is apparent that the floppy (gray) regions have mostly been absorbed into bundles, leaving behind large pores (with the exception of some floppy strands connecting bundled regions). The denser rigid clusters remain mostly undeformed; only the percolating cluster has undergone some necessary deformations to accommodate the formation of bundles.

The images in figures 4.6 and 4.21 are typical representations of the simulation results we analyzed. In the following, we will analyze in more detail how the formation of bundles and pores is influenced by the two principal control parameters, the network density $q$ and the capillary interaction strength $\beta$.

\subsection{Morphological Transitions}

In order to quantify the formation of pores observed in wet fiber networks, we employ the maximum covering radius transform described in [MMJ ${ }^{+} 08$ ]. This is a method to analyze pore sizes, and is used, for example, with confocal microscopy reconstructions of fiber networks. 


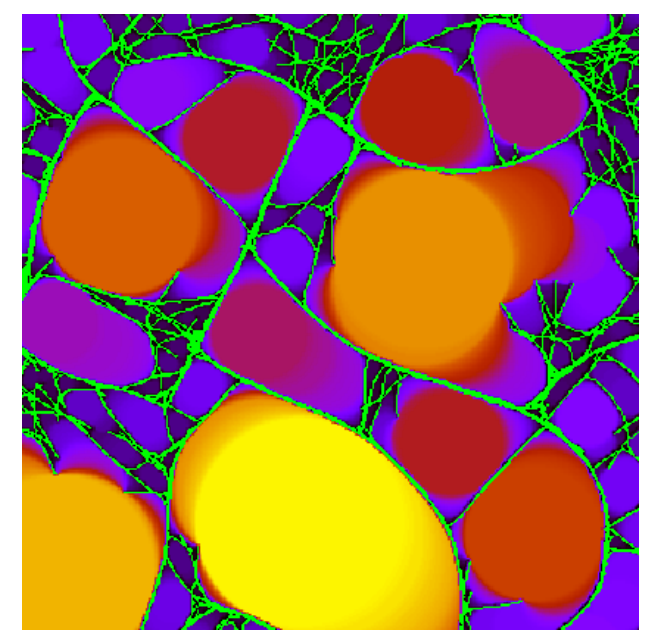

Figure 4.22: Covering Radius Transform of a wet fiber network (detail). Color encodes the maximum covering radius; Fibers are green for better visibility.

The network is first digitized into square cells (we use a set of $1000 \times 1000$ cells here), where each cell is marked as containing either some fiber material or nothing. Then, the Euclidean distance map is computed. This assigns to each empty cell the value of the Euclidean distance from the center of the cell to the center of the nearest cell occupied by a fiber. From this map, the maximum covering radius transform is obtained by assigning again to each cell a value that corresponds to the largest circle that covers the cell in question and does not cover any cells occupied by a fiber. In other words, out of the set of all circles that have a radius equal to the value of their Euclidean distance map (and therefore touch a fiber tangentially without covering any fibers), the largest one that covers a given cell is chosen. That cell is then assigned the value of the circle's radius. In this way, the maximum covering radius function maps the cells of the system to the associated pore size.

Figure 4.22 shows an example of the covering radius transform function of a relaxed fiber network, in a detailed zoom. The fibers are drawn in green, and the covering radius transform is plotted in a color scale ranging from black to yellow. The covering radius transform finds the largest circles that fit into the pores and measures the pore size distribution by the radii of these circles. As suggested in $\left[\mathrm{MMJ}^{+} 08\right]$, we calculate the histogram of the maximum covering radius function to describe the distribution of pore sizes in the system. 

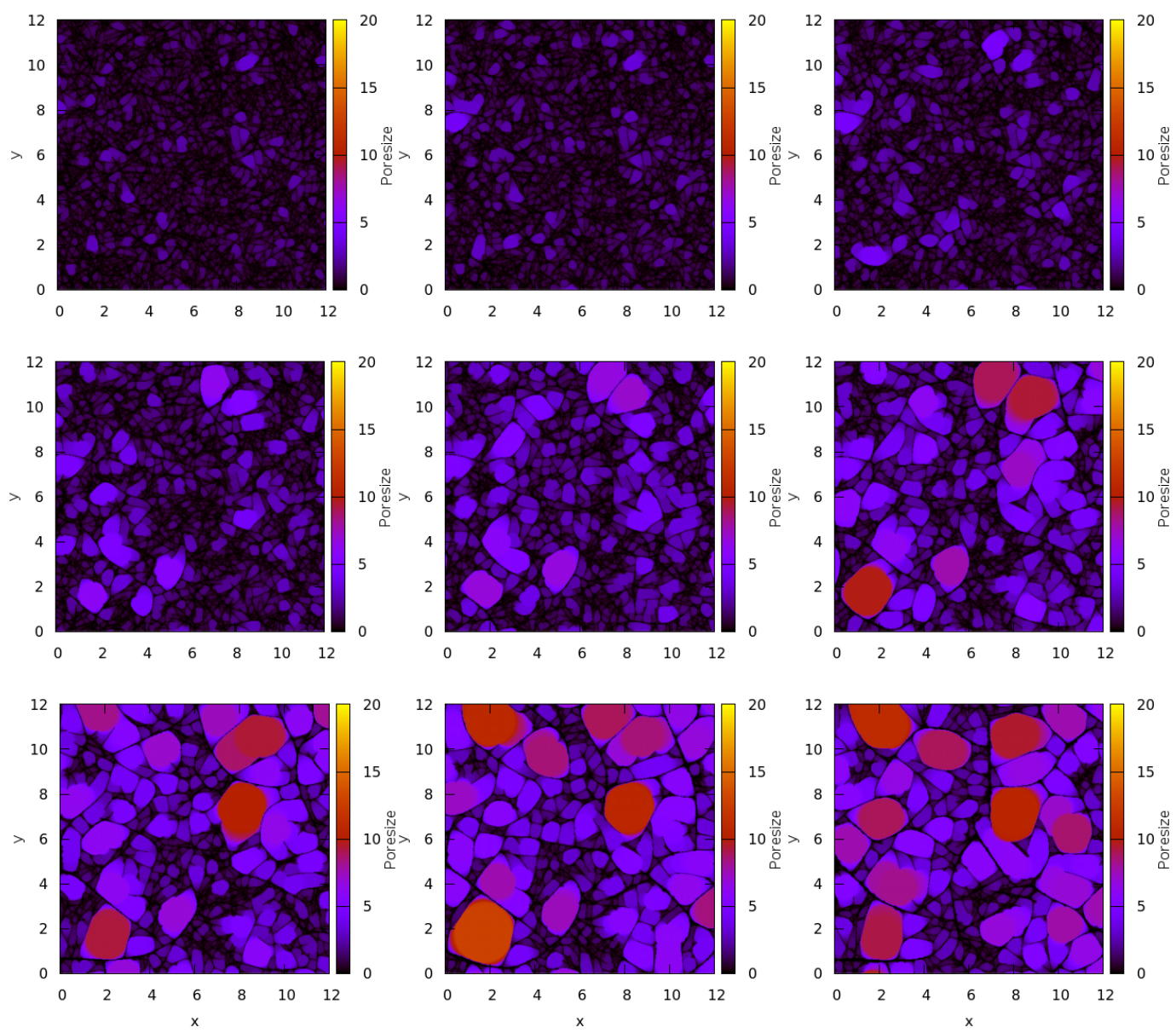

Figure 4.23: Covering Radius Transforms of networks at $q=12.5$, with $\beta$ increasing in equal steps from left to right and top to bottom. The first image is taken at $\beta=0.01$, and the last at $\beta=0.05$, in steps of 0.005 .

Figure 4.23 shows a series of maximum covering radius transform images taken from simulations of a network with a density value of $q=12.5$ and different capillary strengths $\beta=0.01, \ldots, 0.05$. The growth of pores on increasing $\beta$ can be easily observed.

In figure 4.24, the distribution of pore sizes in the system is plotted for various values of $\beta$ as cumulative distribution functions. On increasing $\beta$, some of the smallest pores collapse and are therefore destroyed. Also, a small number of very large pores appears when $\beta$ is increased to large values, and 


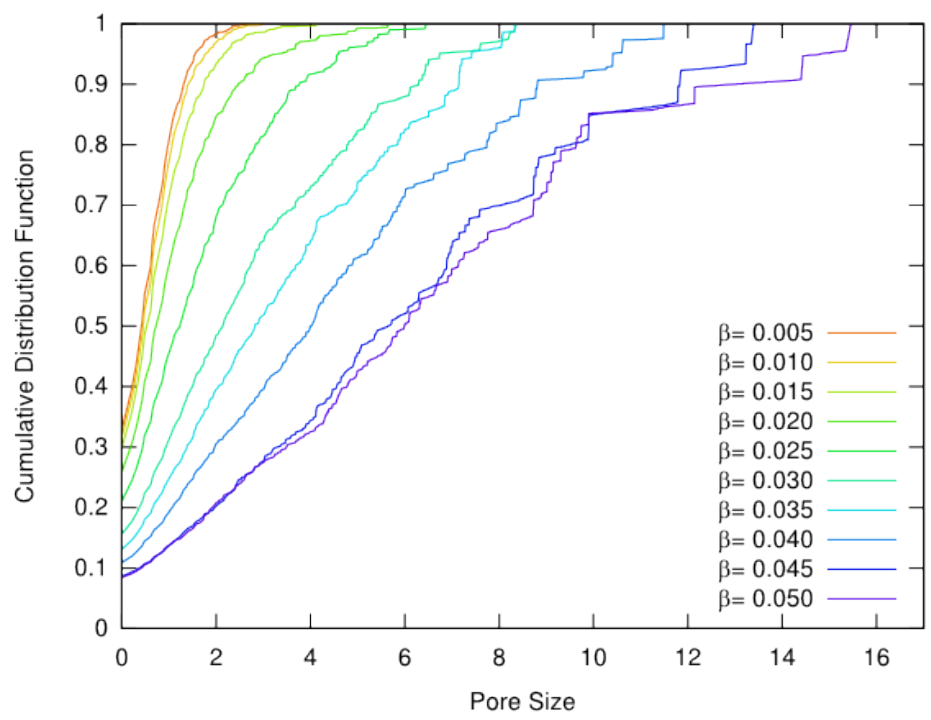

Figure 4.24: Cumulative distribution function of pore sizes in a network of $q=12.5$, for various capillary strengths $\beta$

on the whole the distribution becomes very wide.

Figure 4.25 shows the average value of the pore sizes as a function of $\beta$ for several different networks. Most of the curves follow a characteristic shape: While at low capillary strengths the structure of the network does not change much, there is a certain point where the pores start to grow on increasing the capillary strength $\beta$. At very high capillary strengths, a plateau value is reached and the pore size fluctuates but does not increase further. The point where significant deformation of the network by the liquid bridges starts is shifted to higher capillary strengths for denser networks, while the largest pores found in the plateau region decrease in size for denser networks.

At network densities below $q \approx 10$, our pore size analysis is not exact enough to distinguish the onset point of the linear deformation regime. The curves in figure 4.25 were calculated from simulation results of networks created with the linking probability $p_{l}=0.7$, and using a simplified formulation of the model that does not include stretched liquid bridges. For comparison, the inset shows the same curve calculated with the same simulation protocol as used in the beginning of this chapter. We could identify no significant deviations in the structures and behavior of the networks between the two different simulation methods.

From the pore size data, we extracted the point $\beta_{\text {onset }}$ at which the regime of 


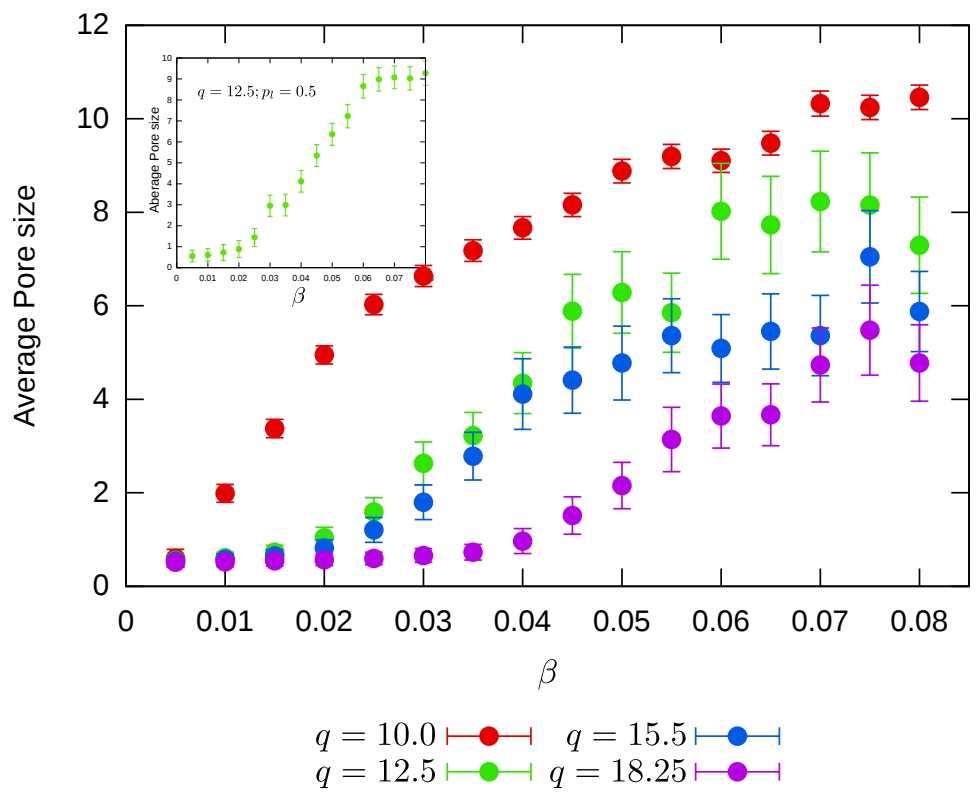

Figure 4.25: Average pore size as a function of the elastocapillary parameter $\beta$ for different crosslinking densities.

linear increase of the pore sizes starts. We did this by fitting a line to the linear regime of each curve, and extrapolating it to the point where the average pore size equals that of the undeformed network. In figure 4.26, these onset values are plotted against $q$. Also marked in the figure are the points of connectivity percolation $q_{c}$ and of rigidity percolation $q^{*}$ for a network of this size $L$ and crosslinking probability $p_{l}$. The points and error bars mark the results of the extrapolation of the onset point from curves of the type as seen in figure 4.25 . Below $q=12$, no such extrapolation was possible due to limited resolution of our pore size analysis. The values show a reasonably linear trend, and extrapolation of this trend to vanishing capillary strengths leads to a network density that lies close to the point of connectivity percolation, and a small but significant distance below the point of rigidity percolation.

Figure 4.27 shows the probability of a fiber being part of the largest connected (blue) or rigid (green) cluster of a network at the same parameters as the networks used to plot figure 4.26. The area where we extrapolated the zero crossing of the line in figure 4.26 to be is at $q=8.1 \pm 0.3$ and is marked in gray. In that region, the network can be expected to have a percolating connected cluster of fibers, but the probability of rigidity percolation is still 


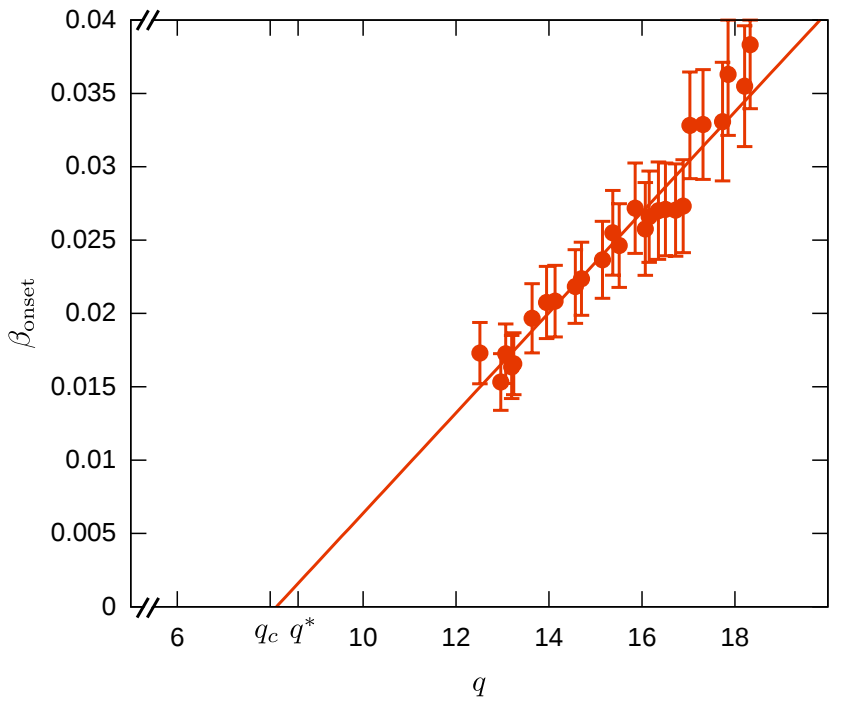

Figure 4.26: Minimum capillary strength at the onset of pore growth as a function of crosslinking density, as obtained from linear fits to pore size curves. A linear fit extrapolates the trend to the point of vanishing capillary strength, which lies between the connectivity percolation point $q_{c}$ and the rigidity percolation point $q^{*}$.

very low.

Below the extrapolated point, already vanishingly small capillary stresses lead to a deformation of the network, meaning that the network becomes floppy below $q \approx 8.1$. Since rigidity percolation of dry networks happens at a somewhat higher density, it is to be concluded that the liquid bridges, connecting some of the non-crosslinked points together and thereby changing the network structure, can make connected but floppy networks rigid.

To address the height of the plateau regions seen in figure 4.25, we plot the average pore size as a function of the density $q$ in figure 4.28. The lines for different capillary strengths $\beta$ appear to converge against a limiting value once the liquid bridges cannot expand the pores any further.

A possible limit to the size of pores in the network can be seen in the structure of the network itself. When a set of connected fibers forms a loop, a pore in the network that is bounded by this loop of connected fibers can only grow further by stretching the bounding fibers. Smaller pores can also grow by rearranging fibers through bending and rotations around the free hinges of the crossing points, but once a pore is bounded by a loop of connected segments, 


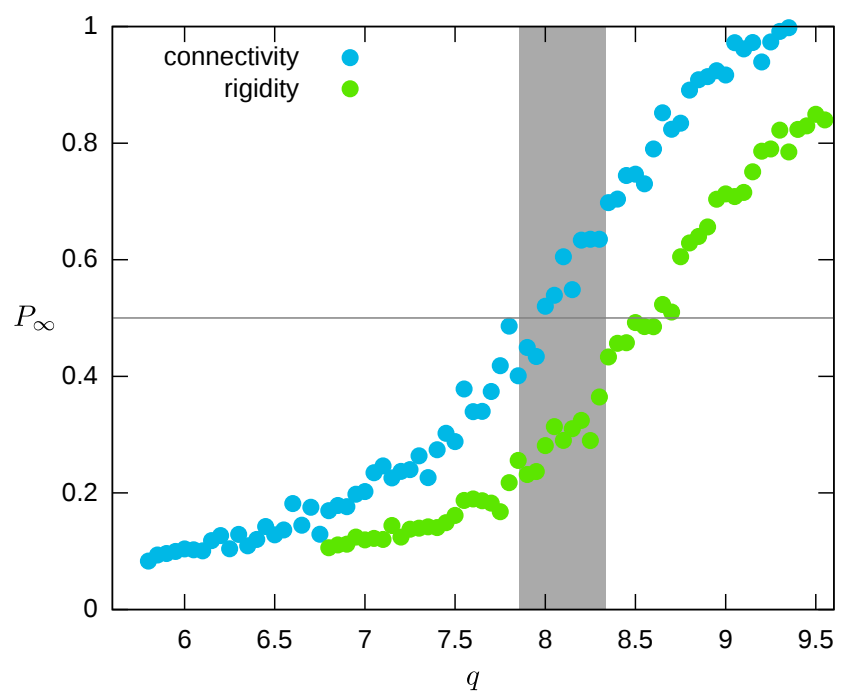

Figure 4.27: Percolation transitions of the connectivity and rigidity of a network at $p_{l}=0.7$ and $L=12$. Plotted are the probabilities of a segment being part of the largest connected (blue) or the largest rigid (green) cluster, as a function of fiber density $q$. The connectivity percolation occurs around $q_{c}=8.0 \pm 0.2$, while rigidity percolates around $q^{*}=8.6 \pm 0.4$. The shaded region corresponds to the $95 \%$ confidence interval of the extrapolated $q_{\text {onset }}$.

further growth of the pore is only possible through stretching deformations, which cost significantly more energy.

Therefore, the pore structure of the network is bounded by the pore size of an ingrained mesh, meaning a mesh of all the loops formed by crosslinked fibers in the system.

We used a graph coloring algorithm to identify the set of connected loops and measure its size distribution. The algorithm is described in appendix A.3. The radii of the loops (defined as half their diameters) are plotted with open symbols in figure 4.28. While the observed pore sizes in the wet networks are still significantly lower than the sizes given by the ingrained mesh, it is still apparent that the ingrained mesh constitutes an upper limit for the pore size.

An alternative morphological quantity of interest is the length of the aligned bundles of fibers that we observe at larger $\beta$. To measure this, we plot the correlation function of the angle between pairs of segments. This is defined as 


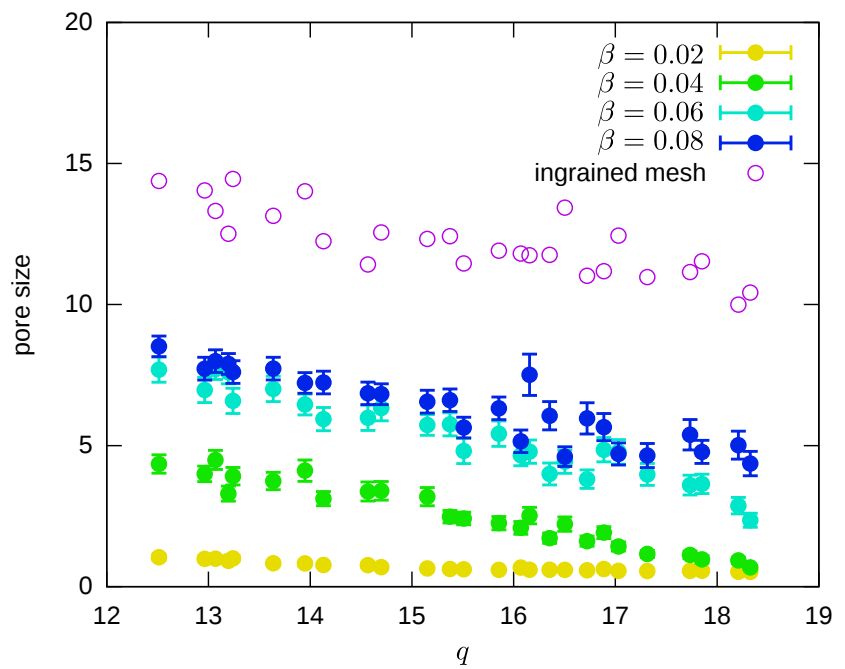

Figure 4.28: Average pore size as a function of the network density $q$, for various capillary strengths $\beta$. The open symbols represent the average sizes of the elementary cycles in the network.

the averages

$$
S(r)=\left\langle\cos ^{2}\left(\Phi_{i}-\Phi_{j}\right)\right\rangle(r)-\frac{1}{2},
$$

where the $\Phi_{i}$ are the orientation angles of the segments, and $r$ is the distance between their centers. The averages are performed over all pairs of segments that do not belong to the same fiber. When fibers are aligned in long bundles, their orientations will be correlated over distances comparable to the bundle length. Figure 4.29 shows plots of these correlation functions on a logarithmic abscissa. They show an exponential decay over a small range of distances, and then drop off quickly when the distance reaches the magnitude of the fiber length $l_{f}$.

In figure 4.30, we plotted the correlation lengths $\lambda$ extracted from fits to the exponential parts of the correlation functions, where they scale as $\exp (r / \lambda)$. The quantity $\lambda$ is a measure of the average length of a bundle of aligned fibers. The curves look very similar to those seen in figure 4.25, but show significantly larger fluctuations, which is why we elected to focus our analysis on the measurements of the pore size. 


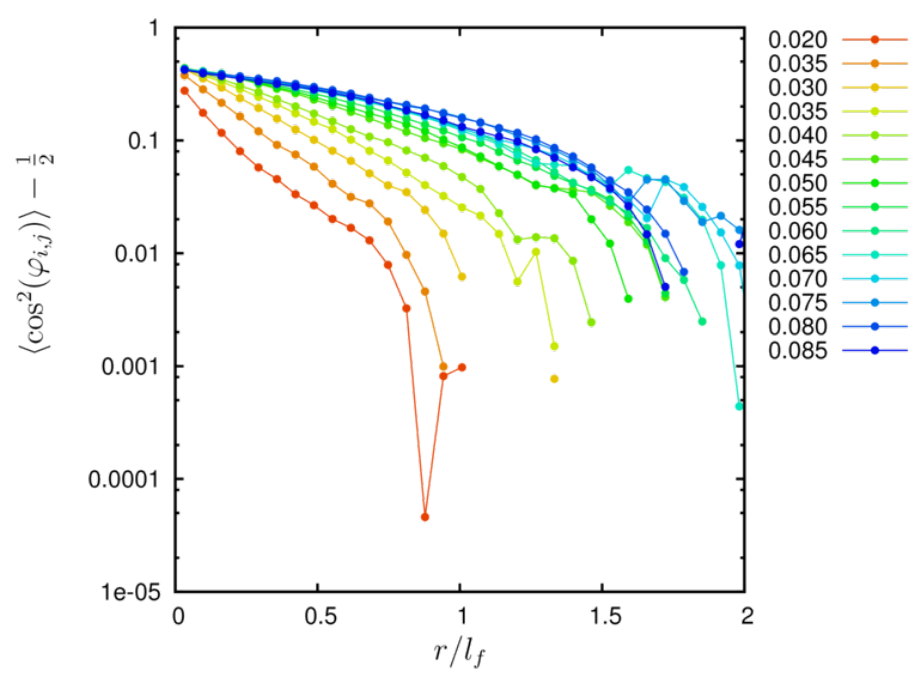

Figure 4.29: Angular correlation functions of fibers in a network at $q=12.5$, for increasing capillary strengths $\beta$.

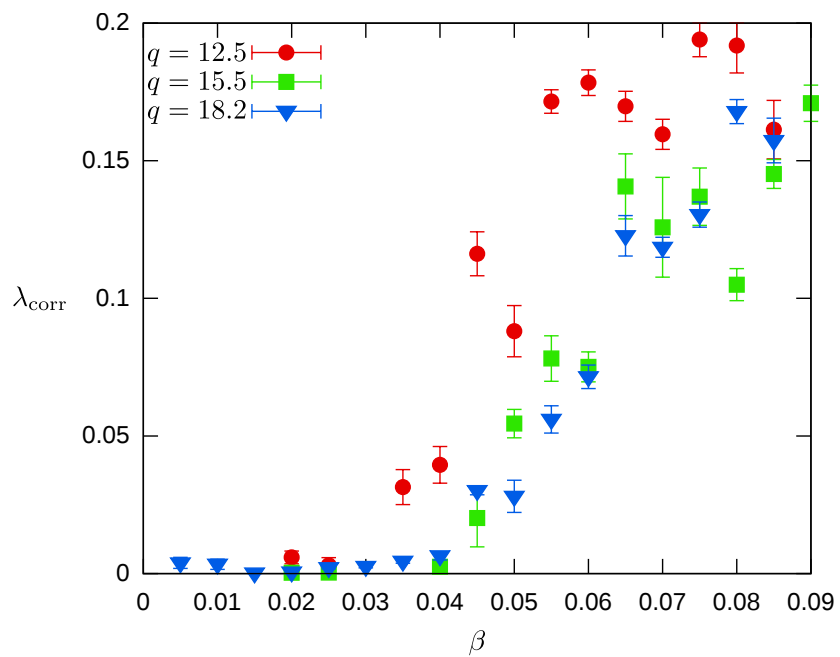

Figure 4.30: Plot of the angular correlation length versus elastocapillary parameter for various crosslinking densities $q$. 


\subsection{Elastic Response}

Figure 4.31 shows the distribution of stresses through the system. It was calculated by summing up the elastic and capillary forces transferred over a set of vertical and horizontal cuts through the simulation box. This enabled us to measure the average values of the stress tensor and their spatial distribution through the system.

As can be observed here, the distributions widen when the capillary strength $\beta$ is increased, meaning that absolute stresses grow larger locally. While the shear stress remains low on average, the average normal stress becomes more negative when the capillary action becomes stronger. This means that the capillary forces drive network to shrink, although actual shrinkage is prevented by the periodic boundary conditions.

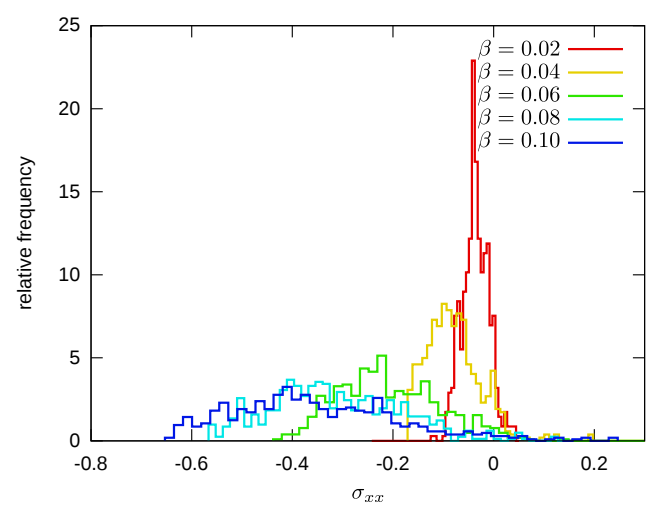

(a)

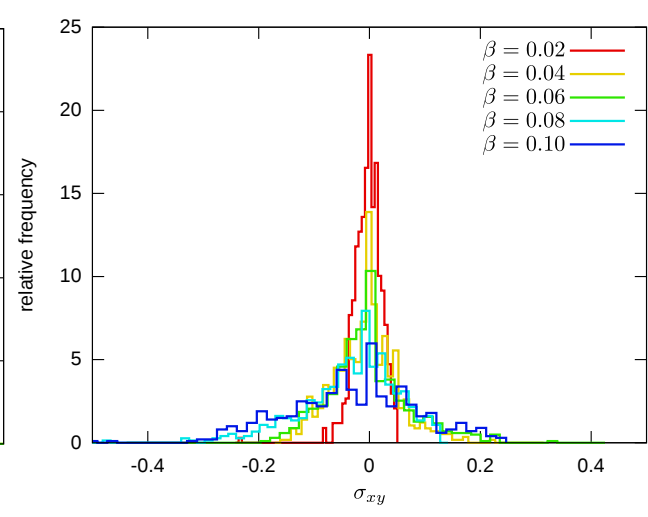

(b)

Figure 4.31: Histograms of the normal (a) and shear (b) components of the stress tensor in a network of density $q=9$, for varying capillary strengths $\beta$. 


\section{Chapter 5}

\section{Conclusions and Outlook}

\subsection{Conclusions}

We set out in this work to develop a model that enables us to analyze the effect that a wetting liquid has on the morphology and structure of a disordered network of elastic fibers. As a starting point, we considered the problem of finding the interfacial shape of a liquid condensing at the crossing point of two smooth and straight cylinders, in chapter 2, In an analytical approach to the problem, we considered two different approximations. The first is based on the theory of Princen [Pri69a, Pri69b, Pri70] and is valid for small crossing angles of the cylinders. The second is valid for arbitrary crossing angles, but only for large negative Laplace pressures. We verified that the asymptotic case of small Laplace pressures is reproduced by the Princen theory, and derived the scaling exponents for the interfacial free energy, the liquid volume, the Laplace pressure, the wetted length and the torque as a function of the crossing angle. We verified these scaling behaviors in numerical minimizations of the free energy, and compared the scaling coefficients derived from numerics and from analytical theory.

As a result, we are able to characterize the capillary torque that arises between the two fibers as a power law with an exponent that depends solely on the thermodynamic ensemble of the wetting problem, and that is valid for a very wide range of crossing angles.

In order to describe the elastic network, we amended the well-known Mikado model to the case of diluted crosslinks. In this way, we are able to allow for rearrangements and transport of liquid through the network. Since this amendment introduces an additional parameter (the crosslinking probability $p_{l}$ ), we investigated the influence of this parameter on the elastic properties of a diluted Mikado network. To do this, we implemented the Pebble Game 
algorithm by Jacobs and Thorpe [JT95] to investigate the topological rigidity of diluted Mikado networks. In this way, we were able to identify rigidly connected clusters. We could reproduce the literature results for the undiluted case, and found a shift in the rigidity percolation with $p_{l}$, accompanied by a qualitative change in the topological properties of rigid clusters in the network.

With these ingredients, we were able to develop a new model suitable for computer simulations of wet elastic networks. In this model, fibers are elastically deformed by liquid bridges at the contact points, which leads to capillary cohesion of the fibers. This is a very effective way to simulate wet fiber systems, since the effect of the wetting liquid is accounted for by only a small number of discrete liquid bridge objects, obviating the need to simulate the fluid dynamic problem of the liquid in parallel.

We had to invest some effort in order to arrive at such a model in which the translational forces and the torques behave consistently. Since the capillary action is governed by the crossing angles, both aligning torques and translational forces arise, the latter being induced by the curvatures of the fibers. We therefore introduced an interpolation scheme to smear out the curvature over the whole of the discretized fiber length, and calculated the resulting forces. Additional force terms arise when liquid bridges lose contact and become stretched. It is of great importance to ensure proper tracking of the loci of liquid bridges, and to account for formation and rupture events. Doing this in a computationally efficient way is the key point to applying this kind of model. While models for wet systems of spheres that take a wetting liquid into account by means of liquid bridge objects are already widely used, the equivalent description for elongated elastic fibers is, to our knowledge, new, and is the main achievement of this work.

Applying this model to Mikado networks, we found that for low interfacial tensions and high elastic moduli of the fibers, the distribution of crossing angles in the system is changed slightly. Once the capillary interaction becomes stronger and crosses a threshold, cooperative effects set in, in which the aligning action of the liquid bridges leads to new fiber crossings, and new liquid bridges appear as a consequence. This leads to bundling of the fibers mediated by large accumulations of liquid bridges, and consequently to the growth of pores in the network. The latter effect is interesting for many applications such as aerosol, where the pore structure influences the efficient, and composite materials, where it influences the mechanical properties.

The threshold value of the capillary interaction strength depends on the 
concentration of fibers in the network. Our investigations show that even below the rigidity percolation point, the presence of liquid bridges organizes the fibers into larger cell structures that give the network a higher rigidity, as evidenced by the fact that the point where vanishingly small capillary forces do not start the cooperative process of bundling anymore lies in the interval between the point of connectivity percolation and that of rigidity percolation.

For the limit case of large interfacial tensions and low elastic moduli, we found that the process of bundling and pore growth is limited by the internal structure of the network, and identified an upper limit of the pore size given by the mesh of connected loops.

\subsection{Outlook}

\subsubsection{Lattice Gas simulations}

We have also taken some preliminary steps to replace the discrete bridge model presented in chapter 4 with a bulk description of the liquid using a lattice gas approach. To this end, we modeled the liquid as an Ising spin-system on a square lattice of $N$ sites, where each spin pointing up represents condensed liquid, and each spin down represents vapor. The lattice has a spacing of width $d$. For each cell of the lattice, the total length of fibers passing through the cell is measured and called $l_{i}$, where the index $i$ runs over all cells. We modified the Ising model to include preferential wetting on the fibers, so that the total energy of the lattice gas reads:

$$
E=-\frac{\epsilon}{2} \sum_{i=1}^{N} \sum_{j \in n_{i}} s_{i} s_{j}-\frac{\epsilon}{2 \sqrt{2}} \sum_{i=1}^{N} \sum_{j \in n n_{i}} s_{i} s_{j}-\delta \sum_{i: s_{i}=+1} l_{i} .
$$

Here, $n_{i}$ indicates the set of the nearest neighbors of cell $i$, while $n n_{i}$ is the set of the four next-nearest, diagonal neighbor cells. The parameter $\delta$ is used to tune the wettability of the fibers, and it results in an effective two-dimensional line tension

$$
\tau=\frac{\epsilon}{d}
$$

Lukas et al. have used a similar model in [LGP97] to simulate fluid imbibition in random fiber assemblies under gravity, without taking elastic deformations of the fibers into account. 


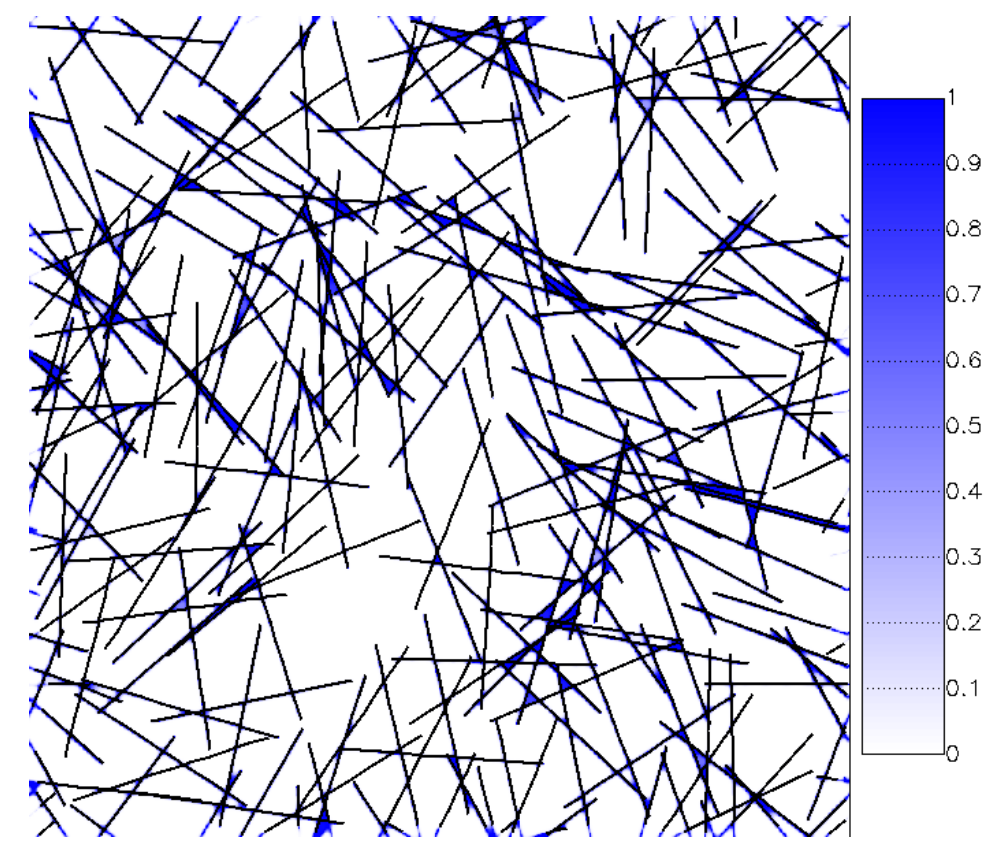

Figure 5.1: Lattice gas simulation of a liquid wetting a Mikado-type random network of infinitely stiff fibers. The system has a size of $800 \mathrm{x} 800$ lattice sites, with the parameters $\eta=\delta=1$, and contains 203 fibers of length $200 d$ and $8 \cdot 10^{4}$ up-spins. The spins were averaged over $10^{9}$ Monte Carlo steps, and the average occupancy of each cell by a positive spin is plotted as the blue color gradient.

The equilibrium state lattice gas system is examined by Monte Carlo sampling, using the Metropolis-Hastings algorithm (see, for example, [FS02]). In particular, we use non-local Kawasaki dynamics. This means that we choose a cell with a spin of +1 (up) and another cell with a spin of -1 (down) at random and attempt to swap the two spin states. Figure 5.1 shows the result of such a sampling of the lattice gas states in the presence of a fiber network. It is similar to the images in [LGP97]. The liquid condenses on the fibers and preferentially fills the small pores at the crossing points.

To extend this type of analysis to elastic fibers, we perform alternate Monte Carlo moves of the spin system and of the fiber network. A Monte Carlo move of the fiber network consists of attempting to displace a randomly chosen node of a fiber to a random point within a circle of radius $d$ of its original position. Afterwards, the total energy of the system, summing over (5.1) and over the elastic energy according to (4.14)-(4.15), is calculated, and the step 


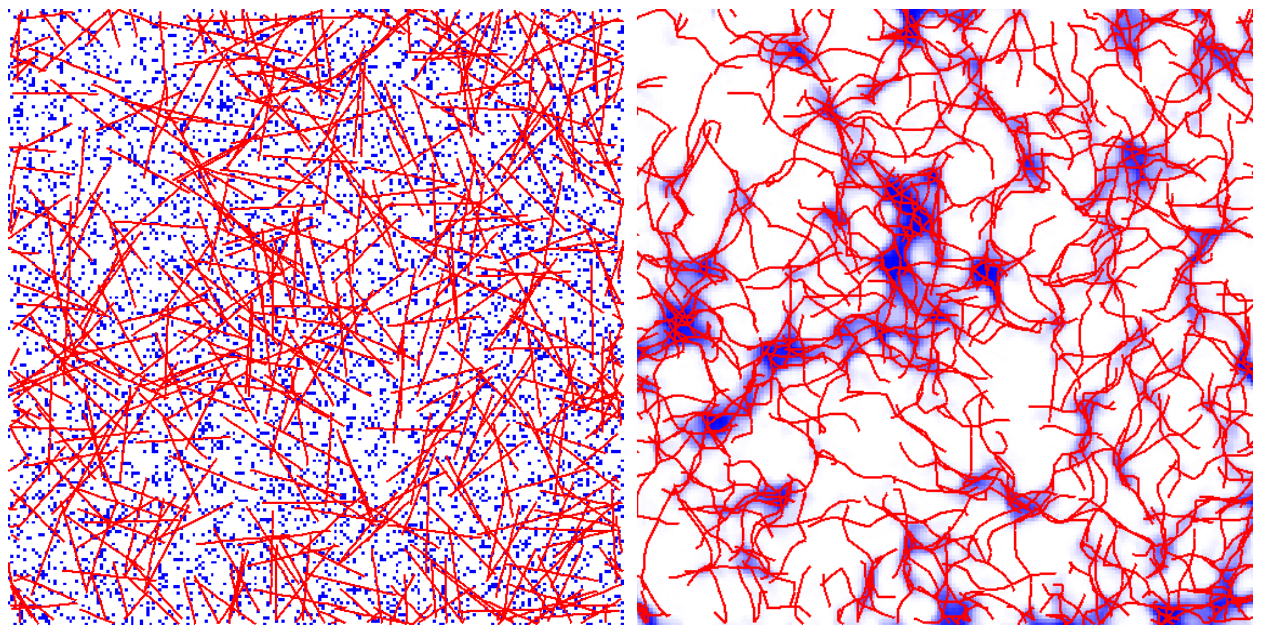

(a) Initial system

(b) Monte-Carlo average

Figure 5.2: An example of a lattice-gas simulation of an elastic wet fiber network. Figure 5.2(a) shows the initial condition of the network (red lines) and the spin states (blue: spin up, white: spin down), while 5.2(b) shows the averaged network configuration and lattice gas state (as a blue-white color gradient). The system parameters are the same as in figure 5.1 .

is accepted according to the Metropolis choice. A prefactor $\zeta$ that weighs the elastic energy contribution is used to control the relative strength of the capillary and elastic energies. This parameter plays the same role as $\beta$ in the Discrete Element Model.

In order to avoid contrived moves of the fibers that would separate a fiber from its already accumulated liquid layer, and therefore be associated with a large energy cost, the spins of the lattice gas system are displaced locally to ensure that the fiber is covered by the same number of up-spins after the move. Special care has to be taken to ensure that these combined moves, moving the liquid and fibers simultaneously, obey the condition of detailed balance.

Figure 5.2 shows the initial state and Monte Carlo sampling of an elastic fiber network embedded in a lattice gas. Since Monte Carlo simulations are thermal, and the network system is in thermal equilibrium with the liquid, the fibers undergo large fluctuations. Nevertheless, it can be observed that bundling of fibers and concentration of the liquid in areas of high fiber con- 
centration still takes place.

Simulations of this kind need a lot of CPU-time, since it takes on the order of $10^{8}$ accepted Monte Carlo steps until the system reaches thermal equilibrium, although the used system sizes with $800 \times 800$ lattice sites and 2000 fiber nodes are already very small. Also, this approach can only describe microscopic fibers that are in thermal equilibrium with the wetting liquid. The class of materials that were described in this thesis, such as textile fibers, paper, or fibrous composites, consist of macroscopic fibers that do not undergo thermal fluctuations. The thermal systems accessible by these Monte Carlo Simulations are rather applicable to crosslinked elastic gels in the presence of a binary liquid. The fluctuations observed in Monte Carlo simulations render morphological analysis of the kind presented in chapter 4 difficult.

However, the fact that a similar coarsening effect of the liquid structure in areas of high fiber density could be observed in simulations that treat the wetting liquid as a bulk object, rather than using discrete liquid bridge elements, corroborates the findings achieved using the discrete model presented in section 4.2.1.

\subsubsection{Lees-Edwards shearing}

In order to drive the system from the outside, we started to apply an external shear via Lees-Edwards boundary conditions, as described already in section 4.3. Since large external shears will drive a number of fibers to alignment, we expect to induce the formation of more liquid bridges that will then form further bundles that cannot be destroyed completely by reversing the shear. Therefore, the system should show hysteretic behavior during a cyclic shearing process.

Figure 5.3 shows a simulation where the shear was increased to a relative shear strain of $\epsilon_{x y}=0.5$ and then reduced back to zero. Plotted are the average shear stress (in green) and the number of liquid bridges (in blue). The shearing builds up a stress in the system that is not completely released. The number of liquid bridges also irreversibly increases. 


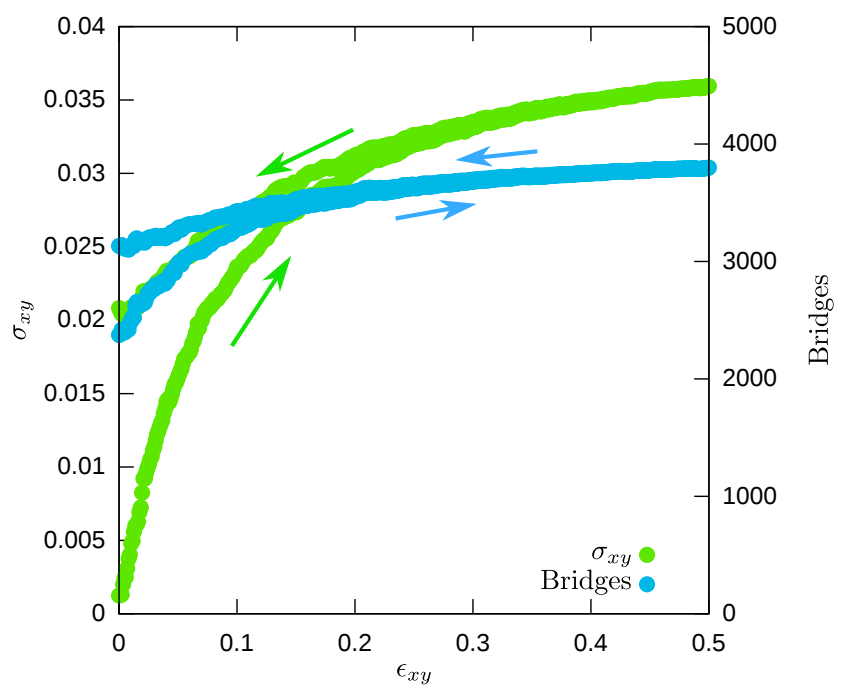

Figure 5.3: Shear stress $\sigma_{x y}$ and number of liquid bridges as a function of applied shear strain $\epsilon_{x y}$ during a cyclic quasistatic shearing. 


\section{Appendix A}

\section{Implementation Details}

\section{A.1 Data Structures}

The following internal data structures are used to represent a (wet) fiber network.

\section{A.1.1 Supernodes}

A Supernode primarily describes a physical position of a point on a given fiber. The following attributes are stored:

- pos: Position $(x, y)$ of the periodic image of the node within the main simulation box.

- real_pos: Absolute position of the node.

- forcesum: Sum of all the forces acting on this supernode (always equals the sum over the forces on its child nodes).

- velocity: Current velocity.

- derivs: Array of vectors storing higher time derivatives of the position for the Gear Algorithm.

- nodes: Pointer to a list of node-Objects. All elements in this list are nodes of fibers crosslinked at the supernode's position.

- xbox/ybox: Integers storing the index of the periodic box copy the supernode lies in (used for Lees-Edwards) 


\section{A.1.2 Nodes}

The class node is derived from the class supernode. It inherits the positions and derivatives from its supernode. It also stores:

- leff: Effective length of fiber mass associated with the node

- leff_naught: Equilibrium value.

- cos_phi: Cosine of the outer angle between the two attached segments

- E: Stored bending Energy

- force: Accumulated Force acting on this node

- connections: Pair of pointers to the attached segment objects.

All functions moving the supernodes take care of updating the relevant subnodes.

\section{A.1.3 Segments}

A segment represents a length of fiber between two nodes. It has the following attributes:

- 1: Length

- 1_naught: Equilibrium length

- E: Stretching Energy

- nodes: Pair of pointers to the node objects at the ends

- cells: Set of pointers to the cells the segment currently occupies

- fiber: Pointer to the fiber object the segment belongs to.

\section{A.1.4 Fibers}

Fiber objects keep track of the individual fibers and provide access to the constituting elements for external algorithms. They provide functions for splitting, adding and deleting segments and traversing the segment list. 


\section{A.1.5 Bridges}

A Bridge object represents a sliding contact between two segments. It holds the following data:

- pos: Position of the periodic image of the crossing

- segments: Pair of pointers to the crossing segments

- (wx1, wy1, wx2, wy2): "winding numbers" giving the number of simulation box lengths in each direction by which the segments 1 and 2 have to be translated to produce a crossing in the periodic simulation box.

\section{A.1.6 Stretched Bridges}

A stretched bridge (sBridge) represents a Bridge whose segments have lost contact (either because one of them was at the end of the fiber and the other has slid off, or because a wedge of two segments has slid off a third, crossing segment). In this case, the liquid doesn't immediately redistribute itself into the liquid reservoir, but rather remains in the form of a liquid bridge between the fibers until it is stretched beyond a certain maximum separation $s_{\max }$. In addition to the information carried by a bridge object, sBridges thus have to store the identities of the node they are attached to, the point $P$ on the connected fiber that corresponds to the smallest distance to the node, and the separation $s$.

\section{A.2 Tracking of Bridges}

\section{A.2.1 Keeping track of bridges in the driver routines}

In order to always have a list of bridges at the current crossing points, keeping track of the motions of these crossing points as well as creation and annihilation events occurring when segments start or cease to cross each other, crossing points of all segments have to be recalculated at each time step.

However, since the cessation of a contact bridge is always associated with the creation of a stretched bridge, simple recalculation of all crossing points does not suffice. The set of all existing bridges has to be tracked, their positions have to be updated, and when their respecting segments cease to cross, a stretched bridge has to be created connecting those segments. At the same 
time, pairs of hitherto non-crossing segments have to be rechecked to track the formation of new contact bridges.

In order to do this effectively, a tree structure keeps track of all segment pairs that are already accounted for by a contact bridge. The cross finding algorithm checks this structure first to avoid recalculating those segment pairs. Since these pairs are stored in a search tree structure, this lookup is associated with a computing time cost proportional to the logarithm of the number of segment pairs. This keeps the cost of this operation reasonable.

The driving algorithms of the molecular dynamics programs call an update routine once for each existing bridge before advancing one time step. Also, the global cross finding algorithm is called to account for new bridges. The following subsection describes how the update routine tracks the fate of a bridge.

\section{A.2.2 Determining the fate of a single bridge}

After each time step, when all the nodes have been moved, it is necessary to recalculate the positions of the bridges. To this end, the function determining whether and where two segments cross is called on the two segments associated with the bridge. If it returns a valid coordinate, this is set as the bridge's current position, then the crossing angle and associated parameters are recalculated and updated as well.

When it is found that the two segments do not cross anymore, a range of possibilities has to be checked in turn to determine the fate of this bridge. These possibilities are checked in order, and any positive finding terminates the search for the fate of the bridge. Therefore, it makes sense to search for events in order of likelihood, with the exception of checks that rely on a previous check having failed.

The possibilities written in italics are optional and are not usually compiled into the code.

- Sliding to a neighboring segment: Both of the neighboring segments (if they exist) of each of the two segments are checked for a crossing with the respective other segments. If one is found, the pointers indicating the segments the bridge is attached to are adjusted accordingly and the bridge is associated with the new segment.

- Sliding to a different segment of the fibers: Since crossing points of fibers that are almost parallel can move very quickly, it is possible that a 
contact bridge traverses several segments during a time step. Therefore, segments further away from the original ones are checked for a new crossing in turn.

- Sliding off an end-segment: When one of the segments is the terminal segment of a fiber and no sliding to a different neighbor could be detected, it is assumed that the bridge traversed the segment to the end of the fiber and has to be transformed into a stretched bridge. The associated segments remain unchanged.

- A kink sliding off a segment: It is checked whether one of the participating segments has a neighbor that also used to cross the same partner segment. If this is the case, the segments formed a kink that has moved away from the one crossing segment. Accordingly, two stretched bridges connecting the middle node to the crossing segment are created, and both original bridges are deleted.

- A kink sliding off a kink: In this special case, the same check as above has to be performed for neighboring segments. The resulting stretched bridges connect the two nodes.

If none of these possibilities turn out to be true in a particular case, an error is logged and the bridge is deleted. If these errors occur too often, more of the optional checks have to be activated. 


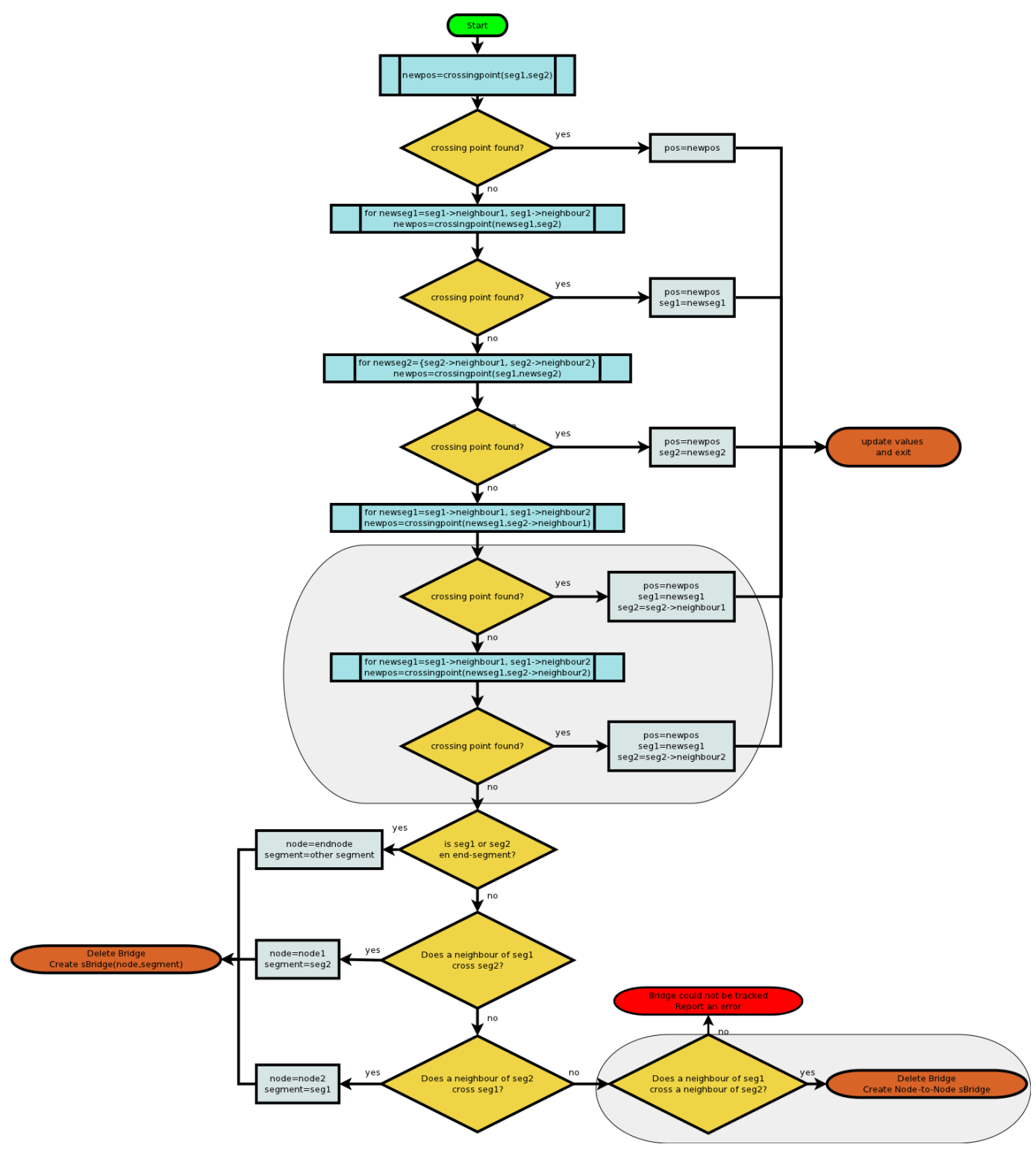

Figure A.1: Flowchart depicting the algorithm that determines the fate of a contact bridge 


\section{A.3 Detection of Elementary Loops}

To analyze the underlying elastic mesh of crosslinked fibers, the set of elementary loops in the graph of crosslinked nodes is determined. To this end, a depth-first spanning tree of the graph is constructed with an edge coloring method as follows:

1. At first, all supernodes are set to color "white". Starting with the first supernode, step 2 is performed recursively with a depth of 0 on all supernodes that are still "white". (This assumes that the graph is connected).

2. The visited supernode is colored "gray" and assigned the current depthvalue. The nearest neighbor nodes are recursively visited with a depthvalue increased by 1 and their predecessors are set to the current supernode. The segment connecting the current supernode and the neighbor is colored "black".

3. After all neighbors have been visited, the node is colored "black".

At this point, the segments and nodes that make up the spanning tree are colored black. The depth-value of each supernode gives the number of steps along the tree from the first supernode, and the predecessor points to the node above in the tree.

All segments colored "white" (i.e. are not part of the spanning tree) define an elementary cycle: If one of these segments is added to the tree, it will contain a cycle. These loops are identified as follows:

1. Start at the node of a white segment that has the lower depth value.

2. Go to the supernode's predecessor and add the connecting segment to the cycle until the white segment's other supernode is reached

This algorithm follows the spanning tree upwards from the end to the beginning of a "white" segment. The result is a set of sets of segments that define loops in the crosslinked network graph. 


\section{List of Symbols}

Chapter 2

$\sigma_{l v}$ surface tension of the liquid-vapor interface

$V_{0}$ Container volume

$(s),(l),(v)$ solid, liquid and vapor phase

$F \quad$ Free energy

$p$ pressure

$\Delta p \quad$ Laplace pressure

$H$ Mean curvature

$p_{\text {sat }}$ Saturation vapor pressure

$r$ fiber radius

$\theta$ Young's contact angle

$\varphi$ see fig. 2.4

$\varepsilon \varphi+\theta-\pi / 2$

$w$ distance of the contact lines (see fig. 2.4)

$R$ radius of curvature of the liquid-vapor interface

$L_{l v} \quad$ contour length of the liquid-vapor interface

$L_{l s}$ contour length of the liquid-solid interface

$A$ cross-sectional area of the liquid filament

$F \quad$ interfacial free energy

$V$ liquid volume

$\alpha$ fiber-fiber crossing angle

$L \quad$ Wetted length

Chapter 3

$\alpha$ fiber-fiber crossing angle

$l_{f}$ fiber length

$L$ system size

$N_{f}$ number of fibers in the system

$N_{c}$ number of crosslinks

$l_{c}$ distance between crosslinks on a fiber 


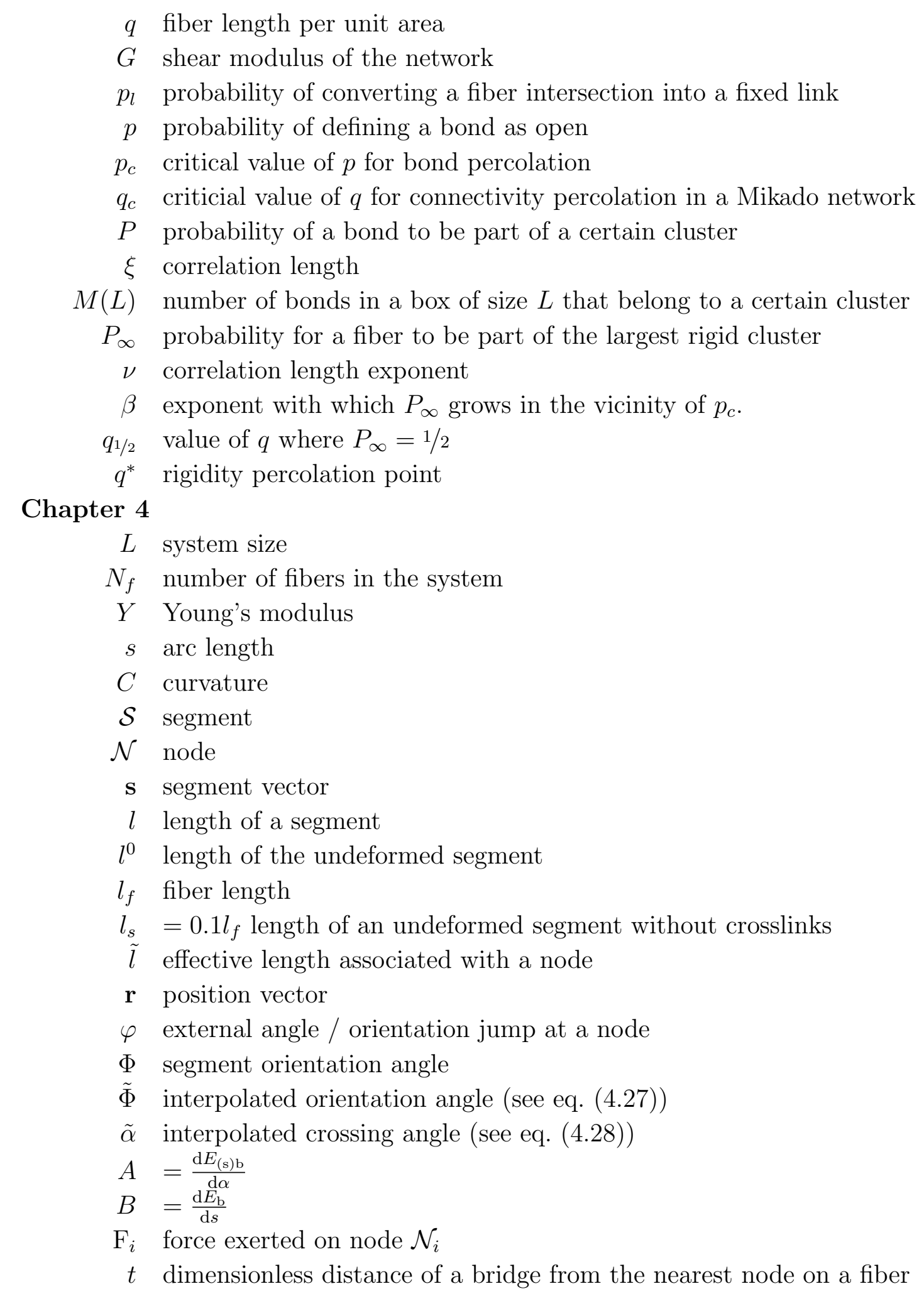


$\beta$ Capillary interaction strength parameter

$\eta$ powerlaw exponent for capillary interaction

$c$ finite segment length cutoff of the liquid bridge potential

$\alpha$ fiber-fiber crossing angle

$\sigma_{l v}$ surface tension of the liquid-vapor interface

$s$ stretch of a stretched bridge

$s^{*} \quad$ stretched bridge rupture distance

$E_{\mathrm{b}} \quad$ energy of a bridge

$E_{\mathrm{sb}} \quad$ energy of a stretched bridge

$r$ fiber radius

$a$ fiber aspect ratio $l_{s} / r$

t tangent vector

$\kappa$ bending rigidity

$\mu$ stretching rigidity

$\varepsilon$ extensional strain

$\varepsilon^{*}$ threshold strain for softcore repulsion $(=0.95)$

$T$ torque

$q$ fiber length per unit area

$\theta$ Young's contact angle 


\section{Bibliography}

[ÅKVK08] J. Åström, P. Kumar, I. Vattulainen, and M. Karttunen. Hardening, avalanches, and strain softening in dense cross-linked actin networks. Physical Review E, pages 1-6, 2008.

[ARD04] Mikko Alava, Martin Rost, and Martin Dubé. Imbibition in disordered media. Advances in Physics, 53(2):83-175, March 2004.

[AT89] M. Allen and D. Tildesley. Computer Simulation of Liquids. Clarendon Press, Oxford, 1989.

[BH57] Simon Broadbent and John Hammersley. Percolation processes I. Crystals and mazes. Proceedings of the Cambridge Philosophical Society, 53:629-641, 1957.

$\left[\mathrm{BHE}^{+} 01\right]$ A. Bausch, U. Hellerer, M. Essler, M. Aepfelbacher, and E. Sackmann. Rapid stiffening of integrin receptor-actin linkages in endothelial cells stimulated with thrombin: A magnetic bead microrheology study. Biophysical Journal, 80(6):2649-2657, June 2001.

$\left[\mathrm{BLL}^{+} 99\right]$ H.W. Beckham, J. Leisen, H.S. Lee, W.W. Carr, S.B. Warner, Esra Coskuntuna, and Fred Kim. Fundamentals of Moisture Transport in Textiles. National Textile Center, (November 1999):1-10, 1999.

[BR02] N.B. Beil and W.R. Roberts. Modeling and Computer Simulation of Compressional Behavior of Fiber Assemblies. Textile Research Journal, 72(4):341-351, 2002.

[Bra92] K. Brakke. The Surface Evolver. Experimental Mathematics, 1(2):141-165, 1992.

[BRMB04] J. Bico, B. Roman, L. Moulin, and A. Boudaoud. Adhesion: Elastocapillary coalescence in wet hair. Nature, 432:690, 2004. 
[BSMM01] Bronstein, Semendjajew, Musiol, and Muehlig. Taschenbuch der Mathematik. Verlag Harri Deutsch, 5 edition, 2001.

[BW09] A. Bedarkar and X.F. Wu. Capillary torque in a liquid bridge between two angled filaments. Journal of Applied Physics, 106(11):113527-113527, 2009.

[CDL ${ }^{+}$93] B.D. Coleman, E.H. Dill, M. Lembo, Z. LU, and I. Tobias. On the Dynamics of Rods in the Theory of Kirchhoff and Clebsch. Archive for Rational Mechanics and Analysis, 121(4):339-359, 1993.

[Cha10] Avik Chatterjee. Nonuniform fiber networks and fiber-based composites: Pore size distributions and elastic moduli. Journal of Applied Physics, 108(6):063513, 2010.

[Cha11] Avik Chatterjee. A simple model for characterizing non-uniform fibre-based composites and networks. Journal of physics. Condensed matter : an Institute of Physics journal, 23(15):155104, April 2011.

[CL89] J. Conder and T. Liew. Fine Mist Filtratoin by Wet Filters-II : Efficiency of Fibrous Filters. Wire, 20(1), 1989.

[Cox52] H. Cox. The Elasticity and Strength of Paper and other Fibrous Materials. British Journal of Applied Physics, 3(MAR):72-79, 1952.

$\left[\mathrm{CST}^{+}\right.$08] Jeffrey Capadona, Kadhiravan Shanmuganathan, Dustin Tyler, Stuart Rowan, and Christoph Weder. Stimuli-responsive polymer nanocomposites inspired by the sea cucumber dermis. Science (New York, N.Y.), 319(5868):1370-4, March 2008.

$\left[\mathrm{CWC}^{+}\right.$04] Nirupama Chakrapani, Bingqing Wei, Alvaro Carrillo, Pulickel M. Ajayan, and Ravi S. Kane. Capillarity-driven assembly of twodimensional cellular carbon nanotube foams. Proceedings of the National Academy of Sciences of the United States of America, 101(12):4009-12, March 2004.

[DH76] H.J. Deuling and W. Helfrich. The curvature elasticity of fluid membranes : A catalogue of vesicle shapes. Journal de Physique, 37(11):1335-1345, 1976. 
[Di192] Ellis Harold Dill. Kirchhoff's theory of rods. Archive for History of Exact Sciences, 44(1):1-23, 1992.

[DML07] M. Das, F. MacKintosh, and A. Levine. Effective medium theory of semiflexible filamentous networks. Physical Review Letters, 99(3), July 2007.

[Dun83] J. Dunlop. On the Compression Characteristics of Fibre Masses. Journal of the Textile Institute, 74(2):92-97, March 1983.

$\left[\mathrm{FGH}^{+} 05\right]$ Z. Fournier, D. Geromichalos, S. Herminghaus, M. Kohonen, F. Mugele, M. Scheel, M. Schulz, B. Schulz, C. Schier, R. Seemann, and A. Skudelny. Mechanical properties of wet granular materials. Journal of Physics-Condensed Matter, 17(9, Sp. Iss. SI):S477-S502, March 2005.

[FS84] S. Feng and P.N. Sen. Percolation on Elastic Networks - New Exponents and Threshold. Physical Review Letters, 52(3):216219, 1984.

[FS02] Daan Frenkel and Berend Smit. Understanding Molecular Simulation. Academic Press, 2 edition, 2002.

[GTV09] T. Gilet, D. Terwagne, and N. Vandewalle. Digital microfluidics on a wire. Applied Physics Letters, 95(1):014106, July 2009.

[GVCB03] M. Gardel, M. Valentine, J. Crocker, and A. Bausch. Microrheology of entangled F-actin solutions. Physical review letters, 91:158302, 2003.

[Her05] Stephan Herminghaus. Dynamics of wet granular matter. Advances in Physics, 54(3):221-261, May 2005.

[HF06a] C. Heussinger and E. Frey. Floppy modes and nonaffine deformations in random fibernetworks. Physical Review Letters, 97(10):105501/1-4, 2006.

[HF06b] C Heussinger and E Frey. Stiff polymers, foams, and fiber networks. Physical Review Letters, 96(1):017802/1-4, January 2006.

[HF07] C. Heussinger and E. Frey. Force distributions and force chains in random stiff fibernetworks. European Physical Journal E, 24(1):47-53, September 2007. 
[HLM03] D. A. Head, A. J. Levine, and F. C. MacKintosh. Distinct regimes of elastic response and deformation modes of cross-linked cytoskeletal and semiflexible polymer networks. Physical Review E, 68(6, Part 1):061907/1-15, December 2003.

[HMFF08] Felix Hoefling, Tobias Munk, Erwin Frey, and Thomas Franosch. Entangled dynamics of a stiff polymer. Physical Review E, 77(6, Part 1):060904/1-4, June 2008.

[HSF07] Claus Heussinger, Boris Schaefer, and Erwin Frey. Nonaffine rubber elasticity for stiff polymer networks. Physical Review E, 76(3, Part 1), September 2007.

[Isr91] Jacob Israelachvili. Intramolecular \& Surface Forces. Academic Press, 1991.

[JT95] D. Jacobs and M. Thorpe. Generic Rigidity Percolation: The Pebble Game. Physical Review Letters, 75(22):4051-4054, November 1995.

[JT96] D. Jacobs and M. Thorpe. Generic rigidity percolation in two dimensions. Physical Review E, 53(4):3682-3693, April 1996.

[Kir59] G. Kirchhoff. Ueber das Gleichgewicht und die Bewegung eines unendlich duennen elastischen Stabes. Journal fï£ jr die reine und angewandte Mathematik, 56:37-67, 1859.

$\left[\mathrm{KTD}^{+}\right.$03] M. Keller, R. Tharmann, M.A. Dichtl, A.R. Bausch, and E. Sackmann. Slow filament dynamics and viscoelasticity in entangled andactive actin networks. Philosophical Transactions of the Royal Society of London Series A-Mathematical Physical and Engineering Sciences, 361(1805):699-711, April 2003.

[Lam70] G. Laman. On graphs and rigidity of plane skeletal structures. Journal of Engineering Mathematics, 4(4):331-340, October 1970.

[LC85] T. Liew and J. Conder. Fine mist filtration by wet filtersâĂŤI. Liquid saturation and flow resistance of fibrous filters. Journal of Aerosol Science, 16(6):497-509, 1985. 
[LCQ04] Elise Lorenceau, Christophe Clanet, and David Quéré. Capturing drops with a thin fiber. Journal of colloid and interface science, 279(1):192-7, November 2004.

[LFXZ07] Jian-Lin Liu, Xi-Qiao Feng, Re Xia, and Hong-Ping Zhao. Hierarchical capillary adhesion of microcantilevers or hairs. Journal of Physics D: Applied Physics, 40(18):5564-5570, September 2007.

[LGP97] D. Lukas, E. Glazyrina, and N. Pan. Computer Simulation of Liquid Wetting Dynamics in Fiber Structures Using the Ising Model. Journal of the Textile Institute, 88:149-161, 1997.

[LHM04] A.J. Levine, D.A. Head, and F.C. MacKintosh. The deformation field in semiflexible networks. Journal of Physics - Condensed Matter, 16(22):S2079-S2088, June 2004.

[LKMT01] M. Latva-Kokko, J. Makinen, and J. Timonen. Rigidity transition in two-dimensional random fiber networks. Physical Review $E$ (Statistical, Nonlinear, and Soft MatterPhysics), 63(4):046113/110, 2001.

[LKT01] M. Latva-Kokko and J. Timonen. Rigidity of random networks of stiff fibers in the low-density limit. Physical Review E (Statistical, Nonlinear, and Soft MatterPhysics), 64(6):066117/1-5, 2001.

[LL86] Landau and Lifshitz. Theory of Elasticity. Elsevier, 3rd edition, 1986.

[Lov44] A.E.H. Love. A Treatise on the Mathematical Theory of Elasticity, volume 105. Cambridge University Press, 1944.

[Max64] James Clarke Maxwell. On the Calculation of the Equilibrium and Stiffness of Frames. Philosophical Magazine, 27:294-299, 1864.

[MBA04] Benjamin Mullins, Roger Braddock, and Igor Agranovski. Particle capture processes and evaporation on a microscopic scale in wet filters. Journal of colloid and interface science, 279(1):213-27, November 2004.

[McH02] G. McHale. Global geometry and the equilibrium shapes of liquid drops on fibers. Colloids and Surfaces A: Physicochemical and Engineering Aspects, 206(1-3):79-86, July 2002. 
[MKJ95] F.C. MacKintosh, J. Kas, and P.A. Janmey. Elasticity of Semiflexible Biopolymer Networks. Physical Review Letters, 75(24):44254428, 1995.

$\left[\mathrm{MMJ}^{+} 08\right]$ Walter Mickel, Stefan Münster, Louise Jawerth, David Vader, David Weitz, Adrian Sheppard, Klaus Mecke, Ben Fabry, and Gerd Schröder-Turk. Robust Pore Size Analysis of Filamentous Networks from Three-Dimensional Confocal Microscopy. Biophysical Journal, 95(12):6072-6080, 2008.

[Mul04] Benjamin Mullins. Effect of fiber orientation on fiber wetting processes. Journal of Colloid and Interface Science, 269(2):449458, January 2004.

[NM99] Z.M. Ning and J.R. Melrose. A numerical model for simulating mechanical behavior of flexiblefibers. Journal of Chemical Physics, 111(23):10717-10726, December 1999.

[NMYO06] R. Nomura, W. Miyashita, K. Yoneyama, and Y. Okuda. Dynamics of capillary condensation in aerogels. Physical Review E, 73(3):1-4, March 2006.

[OH04] Alexander Otten and Stephan Herminghaus. How Plants Keep Dry: A Physicist's Point of View. Langmuir, 20(6):2405-2408, March 2004.

$\left[\mathrm{PBB}^{+} 07\right]$ C. Py, R. Bastien, J. Bico, B. Roman, and A. Boudaoud. 3D aggregation of wet fibers. Europhysics Letters, 77(4):44005, February 2007.

[PKMA09] B. Pokroy, S. Kang, L. Mahadevan, and J. Aizenberg. Selforganization of a mesoscale bristle into ordered, hierarchical helical assemblies. Science (New York, N.Y.), 323(5911):237-40, January 2009.

[PnBR10] Miguel Piñeirua, José Bico, and Benoît Roman. Capillary origami controlled by an electric field. Soft Matter, 6(18):4491, July 2010.

[PRD ${ }^{+}$07] Charlotte Py, Paul Reverdy, Lionel Doppler, José Bico, Benoît Roman, and Charles Baroud. Capillary Origami: Spontaneous Wrapping of a Droplet with an Elastic Sheet. Physical Review Letters, 98(15):156103, April 2007. 
[Pri69a] H. Princen. Capillary phenomena in assemblies of parallel cylinders I. Capillary rise between two cylinders. Journal of Colloid and Interface Science, 30(1):69-75, May 1969.

[Pri69b] H. Princen. Capillary phenomena in assemblies of parallel cylinders II. Capillary rise in systems with more than two cylinders. Journal of Colloid and Interface Science, 30(3):359-371, July 1969.

[Pri70] H. Princen. Capillary phenomena in assemblies of parallel cylinders III. Liquid Columns between Horizontal Parallel Cylinders. Journal of Colloid and Interface Science, 34(2):171-184, October 1970.

[PS74] G. Pike and C. Seager. Percolation and conductivity: A computer study. I. Physical Review B, 10(4):1421-1434, August 1974.

[PV97] Albert Philipse and Alain Verberkmoes. Statistical geometry of caging effects in random thin-rod structures. Physica A: Statistical Mechanics and its Applications, 235(1-2):186-193, January 1997.

[RFD05] D. Rodney, M. Fivel, and R. Dendievel. Discrete modeling of the mechanics of entangled materials. Physical Review Letters, 95(10):108004/1-4, 2005.

[Roe75] R.J. Roe. Wetting of fine wires and fibers by a liquid film. Journal of Colloid and Interface Science, 50(1):70-79, January 1975.

[SA95] D. Stauffer and A. Aharony. Perkolationstheorie eine Einführung. VCH, 1995.

[Saf03] Samuel A. Safran. Statistical Thermodynamics of Surfaces, Interfaces, and Membranes. Westview Press, 2003.

[Ske75a] J. Skelton. Capillary Sorption Hysteresis Losses in Fibrous Assemblies. Textile Research Journal, 45(7):540-542, July 1975.

[Ske75b] J. Skelton. Interfiber forces during wetting and drying. Science, 190(4209):15-20, 1975.

[SL90] Udo Seifert and Reinhard Lipowsky. Adhesion of vesicles. Physical Review A, 42(8):4768-4771, October 1990. 
[TCB06] R. Tharmann, M. Claessens, and A. Bausch. Micro- and macrorheological properties of actin networks effectively crosslinked by depletion forces. Biophysical Journal, 90(7):2622-2627, April 2006.

[TCB07] R. Tharmann, M Claessens, and A. Bausch. Viscoelasticity of isotropically cross-linked actin networks. Physical review letters, 98:088103, 2007.

[VHB09] Alexander Virozub, Nir Haimovich, and Simon Brandon. ThreeDimensional Simulations of Liquid Bridges between Two Cylinders: Forces , Energies , and Torques. Langmuir, 88(10):1283712842, 2009.

[WGR ${ }^{+}$44] I.Y. Wong, M.L. Gardel, D.R. Reichman, E.R. Weeks, M.T. Valentine, A.R. Bausch, and D.A. Weitz. Anomalous diffusion probes microstructure dynamics of entangled F-actin networks. Physical Review Letters, 92(17):178101/1-4, 2004.

[Wyk48] C.M. Wyk. Note on the compressibility of wool. J. of the Textile Institute, 39:285-291, 1948.

$\left[\mathrm{XHL}^{+} 10\right]$ Donghua Xu, Jennifer L. Hawk, David M. Loveless, Sung Lan Jeon, and Stephen L. Craig. Mechanism of Shear Thickening in Reversibly Cross-Linked Supramolecular Polymer Networks. Macromolecules, pages 3556-3565, 2010.

[You05] T. Young. An Essay on the Cohesion of Fluids. Philosophical Transactions of the Royal Society of London, 95:65-87, January 1805.

$\left[\mathrm{ZBH}^{+} 10\right]$ Yongmei Zheng, Hao Bai, Zhongbing Huang, Xuelin Tian, FuQiang Nie, Yong Zhao, Jin Zhai, and Lei Jiang. Directional water collection on wetted spider silk. Nature, 463(7281):640-3, February 2010 . 


\title{
Curriculum Vitae / Lebenslauf
}

\author{
Jann Ohle Claussen \\ Adresse: \\ Papendiek 24 \\ 37073 Göttingen \\ Telefon: (05 51) 3057992 \\ EMail: ohle.claussen@ds.mpg.de \\ Persönliche Angaben \\ Geburtsdatum 10.05.1981 \\ Geburtsort Leer \\ Staatsangehörigkeit Deutsch

\section{Bildungsweg}

2000-2001

"FiPS" (Früheinstieg ins Physikstudium), Fernstudium der Universität Kaiserslautern für Zivildienstleistende

2001-2007 Physikstudium, Georg-August-Universität Göttingen

$2005-2007$ Diplomarbeit, Georg-August-Universität Göttingen

Mai 2007 Abschluss des Diploms

2007-heute Promotion, Max-Planck-Institut für Dynamik und Selbstorganisation, Göttingen

2007-heute Promotionsstium in Physik, Universität Göttingen 


\section{Danksagung (acknowledgements)}

An dieser Stelle möchte ich mich bei den vielen Menschen bedanken, die mir in der Zeit meiner Promotion geholfen und diese Dissertation erst möglich gemacht haben.

Zunächst gilt mein Dank Prof. Stephan Herminghaus dafür, dass er mir die Möglichkeit gegeben hat, ein interessantes Promotionsprojekt in der Abteilung Dynamik komplexer Fluide durchzuführen, in der immer eine ganz besondere kreative Arbeitsathmosphäre herrscht.

Mein besonderer Dank gilt Dr. Martin Brinkmann für das interessante Thema, die kompetente Betreuung meiner Arbeit und dafür, dass es ihm nie an guten Ideen mangelt.

Ausserdem danke ich Prof. Marcus Müller für sein freundliches Entgegenkommen bei der Übernahme des Korreferats.

Für das aufmerksame Korrekturlesen der Arbeit danke ich vor allem Iris Claussen und Martin Brinkmann.

Schließlich danke ich noch einmal besonders meiner Frau Iris Claussen dafür, dass sie mich in der Endphase der Arbeit sehr unterstützt hat, und meinen Eltern Ernst und Helga Claussen für ihren Rückhalt während meines gesamten Studiums. 